\title{
Reduced Partition Function Ratio in the Frequency Complex Plane: A Mathematical Approach
}

\author{
Jie Yuan \\ Key Laboratory of Earth and Planetary Physics, Institute of Geology and Geophysics, Chinese Academy of \\ Sciences, Beijing, China \\ Email: yuanjie@mail.iggcas.ac.cn
}

Received 25 September 2014; revised 20 October 2014; accepted 18 November 2014

Copyright (C) 2014 by author and Scientific Research Publishing Inc.

This work is licensed under the Creative Commons Attribution International License (CC BY). http://creativecommons.org/licenses/by/4.0/

c) (i) Open Access

\begin{abstract}
This paper gives a mathematical approach to calculate the fractionation factor of isotopes in a general cluster (also known as super-molecule), which composes of necessary chemical effect within three bonds outside the interested atom(s). The cluster might have imaginary frequencies after being optimized in quantum softwares. The approach includes the contribution of the difference, which is resulted from the substitution of heavy and light isotopes in the cluster, of vibrations of imaginary frequencies to give precise prediction of isotope fractionation factor. We call the new mathematical approximation "reduced partition function ratio in the frequency complex plane $\left(\mathrm{RPFR}_{C}\right)$ ". If there is no imaginary frequency for a cluster, $\operatorname{RPFR}_{C}$ is simplified to be Urey (1947) or Bigeleisen and Mayer (1947) formula. Final results of this new algorithm are in good agreement with those in earlier studies.
\end{abstract}

\section{Keywords}

Isotope Fractionation, Cluster, Reduced Partition Function Ratio, Frequency Complex Plane

\section{Introduction}

In 1933, Urey and Rittenberg [1] pointed out that the isotopic fractionation factor in different systems could be calculated from spectroscopic data. A more convenient method for the calculation is known as Urey (1947) [2] or Bigeleisen and Mayer (1947) [3] model. This model needs only frequencies to calculate the factor by an equation namely "reduced isotopic partition function ratio (RPFR or $\beta$ factor)". However, a practical problem arises, when a cluster (cut from a big system; see details in Section 2.1) has some imaginary frequencies in the sets of 
vibrational frequencies [4], RPFR cannot evaluate the isotope fractionation factor since it does not deal with imaginary frequencies (see details in Section 2.2).

To overcome this difficulty, Rustad et al. (2008) [4] applied the partial Hessian vibrational analysis (PHVA) [5] in the carbonate (e.g., calcite, aragonite and magnesite) clusters to predict the distributions of isotopes in these minerals; this operation neglects all imaginary frequencies (as well as some real ones) and then the remainder of real frequencies in the sets are used in RPFR, giving the carbon isotope fractionation factors in these minerals. But when using PHVA, also neglected is the contribution of the differences (due to the substitution of heavy and light isotopes in clusters) of imaginary frequencies to the isotope fractionation effect [6]. Therefore, previous problem is still under debate.

This study gives a new approach, i.e. reduced partition function ratio in the frequency complex plane $\left(\mathrm{RPFR}_{\mathrm{C}}\right)$, to the calculation of isotope fractionations in general clusters. This new approach involves a more detailed physical figure of atom vibrations for the calculation than PHVA did; that is, the vibrations of all atoms due to the substitution of heavy and light isotopes in clusters are included to predict the isotope fractionations. This new approach is finally tested by studying isotope fractionation factors in liquid and mineral phases.

\section{Theory}

\subsection{General Cluster for Isotope Research}

We firstly give the theoretical background on building a general cluster for isotope research. The general cluster (Figure 1) includes three parts: A) interested isotopic atom(s) of an element at specific position; B) atoms linking three chemical bonds outside the interested atom(s). Stern and Wolfsberg (1966) [7] had theoretically proved that the biggest necessary influence of chemical effects on an interested isotope is within three bonds; and C) atoms to make the system to be converged in softwares. This kind of cluster could model isotope fractionations in both liquid and solid phases. In practical, researchers cut off atoms from a large periodical system to form solid-phase (e.g., calcite and aragonite in Ref. [4]) clusters, and terminate the outside-broken bonds in part $B$ with some hydrogen atoms in part $C$. For liquid phase, one adds few water molecules (and sometimes few ions [8]) around the interested isotope to simulate its water environments; this technique is also called as "water-droplet" method [4] [9] [10]. For convenience, we represent the general cluster as a super-molecule $X A_{p}$, where $A$ and $X$ represent the interested atom and all atoms in parts of both $B$ and $C$ respectively and the subscript $p$ the number of interested atoms of the same element in the center of the cluster (Figure 1(b)).

\subsection{Harmonic Frequencies in Complex Plane}

As discussed above, the super-molecule is sufficient to describe the chemical influence on isotopes at interested position; then one can use $a b$ initio molecular orbital theory to get the frequencies. In Ref. [11], mass-weighted

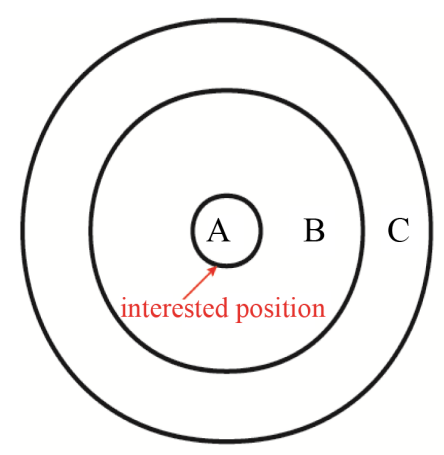

(a)

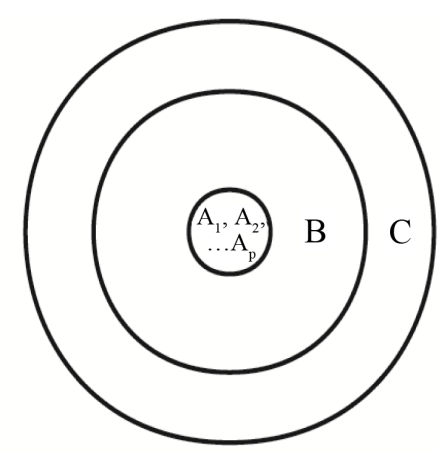

(b)

Figure 1. (a) 2-D schematic diagram of general cluster/super-molecule $X A$ for isotope research. A represents the isotope locates at the interested position, i.e. the center of the cluster; $X$ represents all atoms in $B$ and $C$. (b) One more general cluster $X A_{p}$, with $p$ interested atoms $\left(A_{1}, A_{2}, \cdots, A_{p}\right)$ in the center. See details in the text. 
force constants are defined by

$$
f_{j k}^{\prime}=f_{j k} m_{j}^{-1 / 2} m_{k}^{-1 / 2}
$$

where $m_{k}$ is the mass of the $k$ th atom in the molecule, and the force-constant $f_{j k}$ is the second energy derivatives for coordinates $R_{j}$ and $R_{k}$. And the $k$ th normal-mode displacements has the form

$$
r_{k}=a_{k} \exp (2 \pi v i t)
$$

where

$$
\sum_{j} f_{j k}^{\prime} a_{j}=4 \pi^{2} \omega^{2} a_{k}
$$

in which $4 \pi^{2} v^{2}$ are the eigenvalues of the matrix $f_{j k}^{\prime}$, and $\omega^{\prime}$ are the harmonic frequencies (Hz). This equation gives one set of frequencies for the heavy-isotope system and another for the light-isotope system; these two sets of frequencies are used to calculate the isotope fractionation factor.

The two sets of harmonic frequencies for a super-molecule would, however, sometimes have imaginary frequencies [12]. This is due to the fact that one cannot find a local minimal on the potential energy surface for all atoms of the cluster. And there will be some minus force constants in Equation (1). Upon taking the square root of the left hand side of Equation (3) for a minus mass-weighted force constant, a factor of complex unit $i$ will emerge, and there will be some imaginary frequencies for the molecule. Under this case, one cannot use RPFR to calculate the distribution of isotopes in the super-molecule, because only real frequencies are suitable for RPFR.

For a super-molecule, we suggest that all frequencies, especially the imaginary ones, should be included in the calculation of isotope fractionation. The reasons come from the following facts [11]: for a random frequency $\omega_{k}$, Equations (2) and (3) give the displacement ( $a_{k}$ and $a_{j}$ ) of each and every atom in the cluster. In other words, it gives a very important physical figure: all atoms in cluster will have a motion (with amplitude $a_{j}$ ) for frequency $\dot{\omega}_{k}$. From this point of view, even an imaginary frequency has motions of all atoms in the molecule, and it will affect the difference of the isotope fractionation as vibrational contribution (see the next subsection).

In mathematics [13], each set of frequencies has characteristic properties. The frequencies can be plotted on the complex plane (Figure 2), which is a geometric representation of the complex numbers established by the real axis and the orthogonal imaginary axis. For a general super-molecule, the eigenvalues of the mass-weighted matrix will have $3 N$ frequencies, which might include $n$ imaginary ones, 6 (5) (6, for nonlinear molecular, 5, for linear and diatom molecular) zeros (corresponding to translations and rotations), and $3 N-n-6(5)$ real ones. All non-zero frequencies locate on those two axes, and the zeros at the origin. A real frequency equals its own modulus, i.e. $\dot{\omega}_{\text {real }}=\left|\dot{\omega}_{\text {real }}\right|$; and an imaginary one equals its own modulus multiplying the unit of complex number, i.e. $\omega_{\text {imaginary }}=\left|\dot{\omega}_{\text {imaginary }}\right| i$.

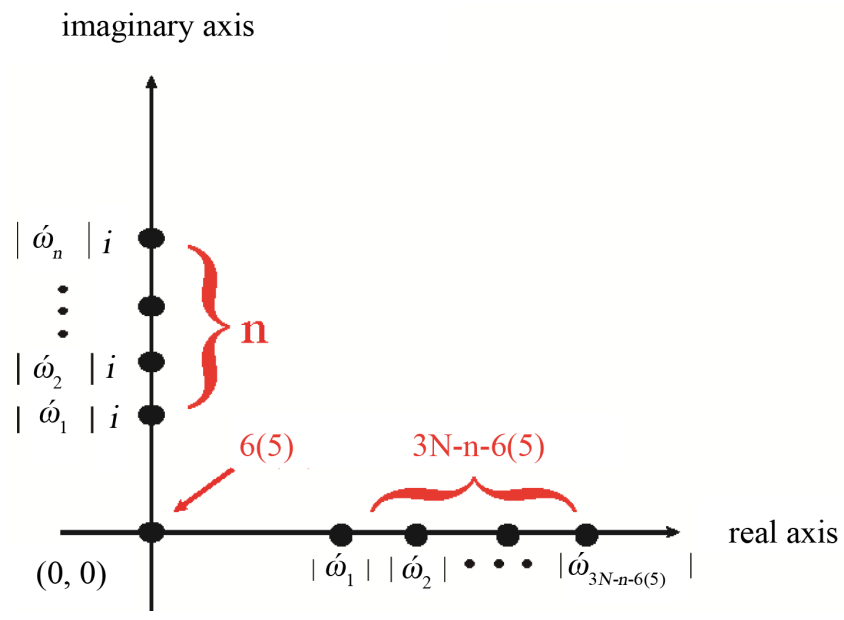

Figure 2. Plots of frequencies for one cluster/super-molecule on the complex plane. 


\subsection{Evaluation of Partition Function and Free Energy of the Super-Molecule}

Based on the Born-Oppenheimer approximation (i.e. nearly harmonic approximation) [14], the translational and rotational, and vibrational energies are the main contribution to the difference of isotope exchange reactions [2] [3]. The followings discuss the partition functions of these three kinds of energies and give the total free energy for the super-molecule.

The translational and rotational energies are in the form of $E_{\text {trans }}=\frac{h^{2}}{8 M}\left(\frac{n_{x}^{2}}{a^{2}}+\frac{n_{y}^{2}}{b^{2}}+\frac{n_{z}^{2}}{c^{2}}\right) \quad\left(n_{x, y, z}=1,2, \cdots\right)$ and $E_{\text {rot }}=\frac{h^{2} J(J+1)}{8 \pi^{2} I} \quad(J=0,1,2, \cdots)[15]$, which are all real numbers. So the translational partition function for the super-molecule is

$$
Q_{\text {trans }}=V\left(\frac{2 \pi M k_{b} T}{h^{2}}\right)^{3 / 2}
$$

where $V$ is the volume of the cluster, $M$ is the mass of the cluster, $k_{b}$ is the Boltzmann constant, $T$ is the absolute temperature, $h$ is the Plank constant. And the rotational partition function is

$$
Q_{r o t}=\frac{8 \pi^{2} I k_{b} T}{\sigma h^{2}}
$$

for diatomic and linear molecules

$$
Q_{\text {rot }}=\frac{\pi^{1 / 2}\left(8 \pi^{2} k_{b} T\right)^{3 / 2}\left(I_{A} I_{B} I_{C}\right)^{1 / 2}}{\sigma h^{3}}
$$

for nonlinear molecules, where $\sigma$ is the symmetry number of the molecule, and $I_{A}$ is the moment of inertia with respect to the appropriate principal axis.

The vibrational energy is in the form of $E_{v i b}=\sum_{k=1}^{n} h \omega_{k}\left(v_{i}+\frac{1}{2}\right)$ (each $\left.v_{i}=0,1,2, \cdots\right)$; but the imaginary frequencies cannot be included in this expression and the partition function in the classical mechanism [15]. However, as shown in previous subsection, this study needs to introduce the contribution of all imaginary frequencies into the partition function and free energy to calculate the isotope fractionation factor. Thus, only for isotope research in ab initio studies, we suggest the vibrational partition function of the super-molecule to be

$$
Q_{v i b}^{*}=\prod_{k=1}^{3 N-6(5)} \frac{\mathrm{e}^{-\frac{h c\left|\dot{\omega}_{k}\right|}{2 k_{b} T}}}{1-\mathrm{e}^{-\frac{h c\left|\dot{\omega}_{k}\right|}{k_{b} T}}}
$$

where $\left|\dot{\omega}_{k}\right|$ is the modulus of the $k$ th frequency from Equation (3). If $\omega_{k}$ is real, $Q_{v i b k}^{*}$ is the vibrational partition function; and if $\omega_{k}$ is imaginary, $Q_{v i b k}^{*}$ is defined as imaginary-frequency correction to the vibrational partition function. Thus we call $Q_{v i b}^{*}$ the imaginary-frequency-corrected vibrational partition function. Furthermore, the imaginary-frequency-corrected Helmholtz free energy of this super-molecule is given by

$$
F^{*}=-R T \ln \left(Q^{*}\right)
$$

where $R$ is gas constant and

$$
Q^{*}=Q_{\text {trans }} Q_{\text {rot }} Q_{v i b}^{*}
$$

\subsection{Teller-Redlich Product Rule in the Frequency Complex Plane}

In Ref. [16], Equation (3) can also be expressed as

$$
[F G]=[F]^{*} \mu_{1} \mu_{2} \cdots \mu_{N}=\lambda_{1} \lambda_{2} \cdots \lambda_{N}
$$


where $[G]$ is the kinetic energy matrix, $\mu_{k}$ is the reciprocal mass of the $k$ th atom in the molecule, $[F]$ is the force-constant matrix, and

$$
\lambda_{k}=4 \pi^{2} v_{k}^{2}=4 \pi^{2} c^{2} \dot{\omega}_{k}^{2}
$$

Since $[F]$ will be identical for the molecule of different isotopes with the same method (i.e. the same exchange-correlation functional/basis set), now taking Equation (11) into Equation (10) gives

$$
\frac{\dot{\omega}_{1}^{\prime} \cdots \cdots \dot{\omega}_{3 N}^{\prime}}{\dot{\omega}_{1} \cdots \cdots \cdot \dot{\omega}_{3 N}}=\left(\frac{m_{1} m_{2} \cdots m_{3 N}}{m_{1}^{\prime} m_{2}^{\prime} \cdots m_{3 N}^{\prime}}\right)^{1 / 2}
$$

where the superscript " ' " " denotes the molecule with heavy isotopes.

Let us submit the frequencies with complex form. The number $n$ of complex unit $i$ is dependent on left hand side of Equation (3) and right hand side of Equation (1). Because $m_{k}, m_{k}^{\prime}$ are real and nearly the same for one element, and the force constant matrix $f_{i j}$ is identical for a cluster with given method, $n$ are the same in the numerator and denominator of Equation (12). We get

$$
\frac{\left|\dot{\omega}_{1}^{\prime}\right| \cdots\left|\dot{\omega}_{k}^{\prime}\right|\left|\dot{\omega}_{k+1}^{\prime}\right| i \cdots\left|\dot{\omega}_{3 N}^{\prime}\right| i}{\left|\dot{\omega}_{1}\right| \cdots\left|\dot{\omega}_{k}\right|\left|\dot{\omega}_{k+1}\right| i \cdots\left|\dot{\omega}_{3 N}\right| i}=\left(\frac{m_{1} m_{2} \cdots m_{3 N}}{m_{1}^{\prime} m_{2}^{\prime} \cdots m_{3 N}^{\prime}}\right)^{1 / 2}
$$

After the cancellation of $i$, we have

$$
\frac{\left|\dot{\omega}_{1}^{\prime}\right| \cdots\left|\dot{\omega}_{k}^{\prime}\right|\left|\dot{\omega}_{k+1}^{\prime}\right| \cdots\left|\dot{\omega}_{3 N}^{\prime}\right|}{\left|\dot{\omega}_{1}\right| \cdots\left|\dot{\omega}_{k}\right|\left|\dot{\omega}_{k+1}\right| \cdots\left|\dot{\omega}_{3 N}\right|}=\left(\frac{m_{1} m_{2} \cdots m_{3 N}}{m_{1}^{\prime} m_{2}^{\prime} \cdots m_{3 N}^{\prime}}\right)^{1 / 2}
$$

Equation (14) is valid only when $3 N$ motions are vibrational normal modes. We consider those 6 (5) motions, corresponding to translational and rotational motions, convert of low frequency corresponding to weak forces. Then the ratio for the translational frequencies and rotation frequencies can be written as:

$$
\begin{aligned}
& \frac{\left|\dot{\omega}_{T}^{\prime}\right|}{\left|\dot{\omega}_{T}\right|}=\left(\frac{M}{M^{\prime}}\right)^{1 / 2} \\
& \frac{\left|\dot{\omega}_{R}^{\prime}\right|}{\left|\dot{\omega}_{R}\right|}=\left(\frac{I}{I^{\prime}}\right)^{1 / 2}
\end{aligned}
$$

Submitting Equations (15) and (16) into Equation (14), we obtain the Teller-Redlich product rule in the frequency complex plane:

$$
\prod_{k=1}^{3 N-5} \frac{\left|\dot{\omega}_{k}^{\prime}\right|}{\left|\dot{\omega}_{k}\right|}=\prod_{i=1}^{3 N}\left(\frac{m_{i}}{m_{i}^{\prime}}\right)^{1 / 2}\left(\frac{M^{\prime}}{M}\right)^{3 / 2}\left(\frac{I^{\prime}}{I}\right)
$$

for diatom and linear molecules and

$$
\prod_{k=1}^{3 N-6} \frac{\left|\dot{\omega}_{k}^{\prime}\right|}{\left|\dot{\omega}_{k}\right|}=\prod_{i=1}^{3 N}\left(\frac{m_{i}}{m_{i}^{\prime}}\right)^{1 / 2}\left(\frac{M^{\prime}}{M}\right)^{3 / 2}\left(\frac{I_{A}^{\prime} I_{B}^{\prime} I_{C}^{\prime}}{I_{A} I_{B} I_{C}}\right)^{1 / 2}
$$

for nonlinear molecules.

\section{Reduced Partition Function Ratio in Frequency Complex Plane}

The differences for the isotopes in the super-molecule can be written as a typical chemical exchange reaction [2] [3]:

$$
\frac{1}{p} X A_{p}+A^{\prime}=\frac{1}{p} X A_{p}^{\prime}+A
$$

where $A$ and $A^{\prime}$ represent light and heavy isotope respectively, and $p$ is the number of interested atoms in the molecule. 
The equilibrium constant for this reaction is given by

$$
K=\exp (-\Delta G / R T)
$$

Because different isotopes have negligible difference of volume, isotope exchange reactions do not involve significant pressure-volume work [15]. The Gibbs free energy is equivalent to the Helmholtz free energy and we take Equation (8) into Equation (18), $K$ can be written as partition function ratio:

$$
K=\left(\frac{Q^{*}\left(X A_{p}^{\prime}\right)}{Q^{*}\left(X A_{p}\right)}\right)^{1 / p} / \frac{Q\left(A^{\prime}\right)}{Q(A)}
$$

Let us substitute Equations (5)-(8) into Equation (19). For diatom and linear molecules, we have

$$
K=\left(\frac{m(A)}{m\left(A^{\prime}\right)}\right)^{3 / 2}\left(\left(\frac{M\left(X A_{p}^{\prime}\right)}{M\left(X A_{p}\right)}\right)^{3 / 2} \frac{\sigma_{X A_{p}}}{\sigma_{X A_{p}^{\prime}}} \frac{I\left(X A_{p}^{\prime}\right)}{I\left(X A_{p}\right)} \prod_{k}^{3 N-5}\left(\frac{\mathrm{e}^{-u_{k}\left(X A_{p}^{\prime}\right) / 2}}{1-\mathrm{e}^{-u_{k}\left(X A_{p}^{\prime}\right)}}\right) \times\left(\frac{\mathrm{e}^{-u_{k}\left(X A_{p}\right) / 2}}{1-\mathrm{e}^{-u_{k}\left(X A_{p}\right)}}\right)^{-1}\right)^{1 / p}
$$

and for nonlinear molecules,

$$
K=\left(\frac{m(A)}{m\left(A^{\prime}\right)}\right)^{3 / 2}\left(\left(\frac{M\left(X A_{p}^{\prime}\right)}{M\left(X A_{p}\right)}\right)^{3 / 2} \frac{\sigma_{X A_{p}}}{\sigma_{X A_{p}^{\prime}}} \frac{I_{A}\left(X A_{p}^{\prime}\right) I_{B}\left(X A_{p}^{\prime}\right) I_{C}\left(X A_{p}^{\prime}\right)}{I_{B}\left(X A_{p}\right) I_{C}\left(X A_{p}\right)} \prod_{k}^{3 N-6}\left(\frac{\mathrm{e}^{-u_{k}\left(X A_{p}^{\prime}\right) / 2}}{1-\mathrm{e}^{-u_{k}\left(X A_{p}^{\prime}\right)}}\right) \times\left(\frac{\mathrm{e}^{-u_{k}\left(X A_{p}\right) / 2}}{1-\mathrm{e}^{-u_{k}\left(X A_{p}\right)}}\right)^{-1}\right)^{1 / p}
$$

where $u_{k}=h c\left|\dot{\omega}_{k}\right| / k_{b} T$.

Equation (20) can be reduced to a more general expression by using Equation (17):

$$
\operatorname{RPFR}_{\mathrm{C}}\left[X A_{p}\right]=\left(\frac{\sigma_{X A_{p}}}{\sigma_{X A_{p}^{\prime}}} \prod_{k}^{3 N-6(5)} \frac{u_{k}\left(X A_{p}^{\prime}\right)}{u_{k}\left(X A_{p}\right)} \frac{\exp \left[-u_{k}\left(X A_{p}^{\prime}\right) / 2\right]\left\{1-\exp \left[-u_{k}\left(X A_{p}\right)\right]\right\}}{\exp \left[-u_{k}\left(X A_{p}\right) / 2\right]}\right)^{1 / p}
$$

where $\mathrm{RPFR}_{\mathrm{C}}$ is short for reduced partition function ratio in the frequency complex plane.

Obviously, one can see that if the super-molecule is at a local minimal on the potential energy surface (i.e. $n=0$ ), all frequencies locate on the real-axis in the frequency complex plane (Figure 2). In such case, $\mathrm{RPFR}_{\mathrm{C}}$ becomes Urey (1947) or Bigeleisen and Mayer (1947) formula. Due to the fact that the set of real numbers (i.e. frequencies here) is the subset of the set of the complex numbers [13], the set of fractionation factors given by Urey (1947) or Bigeleisen and Mayer (1947) formula (i.e. RPFR) is the subset of the set of fractionation factors

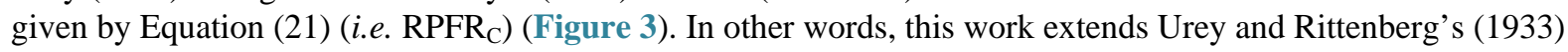
idea [1] to focus on isotope fractionation research in the frequency complex plane.

The fractionation factor between two clusters can be written as:

$$
\alpha=\mathrm{RPFR}_{\mathrm{C} 1} / \mathrm{RPFR}_{\mathrm{C} 2}
$$

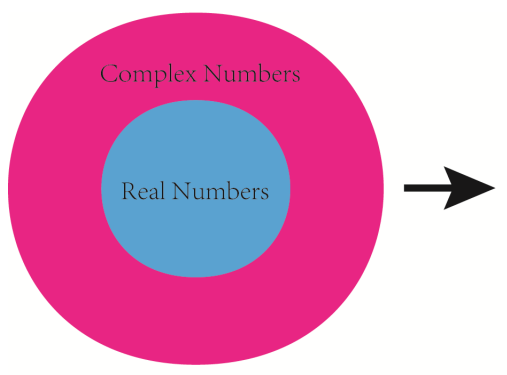

(a)

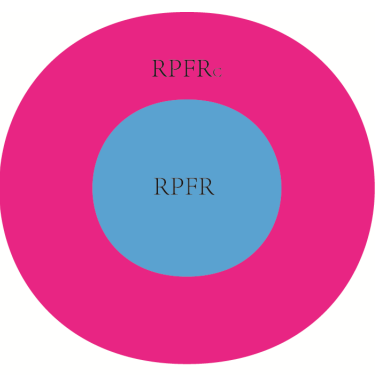

(b)

Figure 3. (a) The set of real numbers (i.e. frequencies here) is a subset of the set of the complex numbers; (b) The set of RFPR is a subset of the set of RPFR $\mathrm{R}_{\mathrm{C}}$. The arrow indicates the process of the calculation of the isotope fractionation factors. Using real frequencies and imaginary ones in the calculations give RPFR and RPFR ${ }_{C}$, respectively. 


\section{Tests of Present Approach}

To understand the new algorithm, we compute RPFR $\mathrm{C}_{\mathrm{C}}$ and/or $\alpha$ in typical isotope systems. Two examples are depicted below not for the accuracy prediction of experimental data, but for the abilities of our algorithm. All frequencies needed in $\mathrm{RPFR}_{C}$ are implemented in Gaussian09 [12]. The optimized geometries and frequencies for all examples are presented in the "Electronic supplementary materials". Present $\mathrm{RPFR}_{\mathrm{C}}$ and $\alpha$ results are compared with corresponding references, i.e. those previously calculated from all real frequencies in published literatures. The difference $\varepsilon$ (in \%) between present result and the reference is in the form of $\varepsilon=\left(\operatorname{RPFR}_{\mathrm{C}} / \operatorname{RPFR}_{\text {ref }}-1\right) * 1000$ or $\varepsilon=\left(\alpha / \alpha_{\text {ref }}-1\right) * 1000$.

1) The geranium isotope fractionation factor $\alpha$ between $\mathrm{GeO}(\mathrm{OH})_{3}^{-1}-\left(\mathrm{H}_{2} \mathrm{O}\right)_{30}$ (Figure 4(a)) and $\mathrm{Ge}(\mathrm{OH})_{4}-\left(\mathrm{H}_{2} \mathrm{O}\right)_{30}$ (Figure 4(b)) (corresponding to $\mathrm{GeO}(\mathrm{OH})_{3}^{-1}-\left(\mathrm{H}_{2} \mathrm{O}\right)_{30}-\mathrm{B}$ and $\mathrm{Ge}(\mathrm{OH})_{4}-\left(\mathrm{H}_{2} \mathrm{O}\right)_{30} \mathrm{D}$ in Ref. [10] respectively) is a good example of study of isotopes in liquid phase. After optimized, each cluster has an imaginary frequency (Table 1). When calculating $\alpha$, Li et al. (2009) neglected the imaginary frequencies because 1) the main vibration vector of this imaginary frequency belongs to a water molecule located at outside of the super-molecule; 2) it is less than $50 \mathrm{~cm}^{-1}$; and 3) RPFR is the same if they neglected it. The values of Li et al.'s $\alpha$ s at different temperatures are taken as references. As shown in Figure 5, the maximum difference $\varepsilon_{\max }$ be-

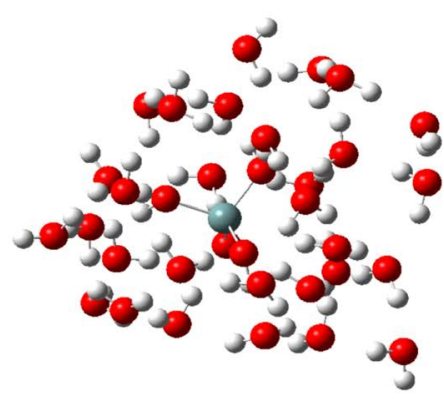

(a)

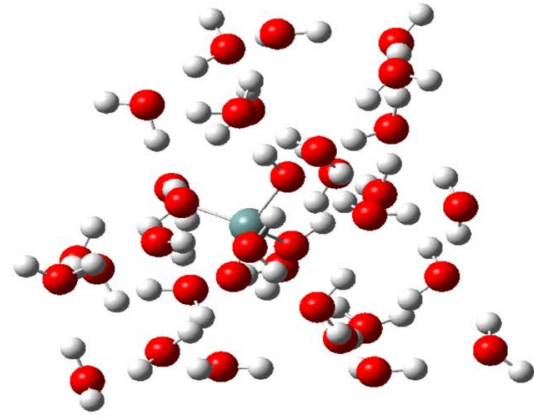

(b)

Figure 4. Water-droplets for a) $\mathrm{GeO}(\mathrm{OH})_{3}^{-1}-\left(\mathrm{H}_{2} \mathrm{O}\right)_{30}$, and b) $\mathrm{Ge}(\mathrm{OH})_{4}-\left(\mathrm{H}_{2} \mathrm{O}\right)_{30}$ (cyan germanium, gray hydrogen, red oxygen). The optimized structures and frequencies are taken from Li et al. (2009).

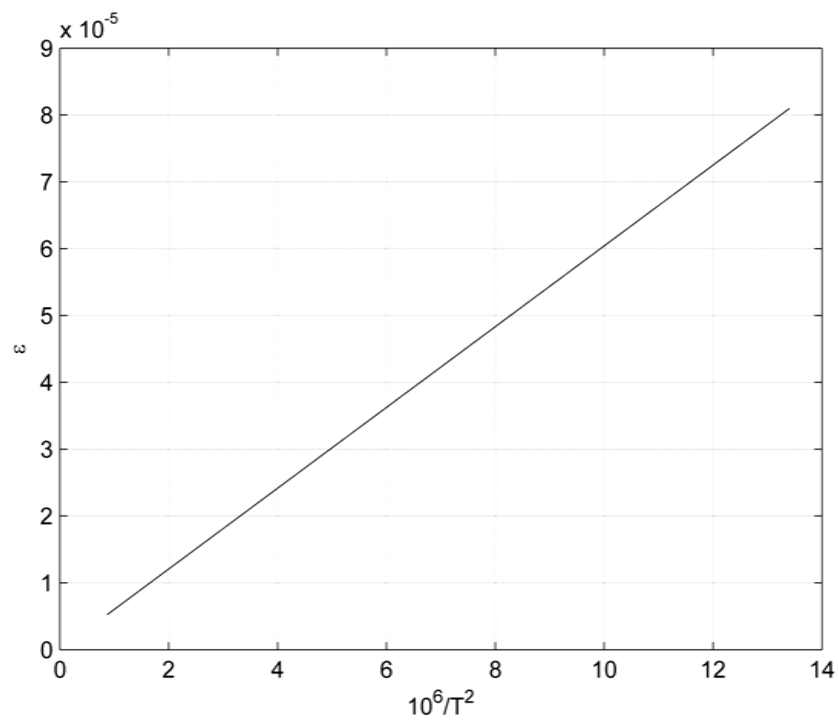

Figure 5. $\varepsilon \alpha \mathrm{Ge}(\mathrm{OH})_{4}-\left(\mathrm{H}_{2} \mathrm{O}\right)_{30}-\mathrm{GeO}(\mathrm{OH})_{3}^{-1}-\left(\mathrm{H}_{2} \mathrm{O}\right)_{30}$ versus $\mathrm{T}(\mathrm{K})$. The corresponding reference $\alpha$ s are from Li et al. (2009). 
tween Li et al.'s and present results is $8.2 \times 10^{-5} \%$ o $(273.15 \mathrm{~K})$; this shows that present approach is very efficient to study isotope fractionation in liquids.

2) The carbon and ${ }^{13} \mathrm{C}-{ }^{18} \mathrm{O}$ clumped isotope fractionations in inner body of calcite are good examples of study of isotopes in solid phase. We cut a cluster (Figure 6) from the periodical calcite, of which the primitive cell parameters (Table 2) are calculated in CRYSTAL06 [17], by the way published in Rustad et al. (2008). The fitted polynomials of $\alpha_{\mathrm{CaCO}_{3} \text {-C }}$ and K3866 in Ref. [18] are taken as references.

Results shown in Figures 7-9 indicate that our new algorithm have high accuracy. For $\alpha_{\mathrm{CaCO}_{3}-\mathrm{C}}$ in Figure 7, $\varepsilon_{\max } \mathrm{s}$ are $-10.2 \%$ o $(273.15 \mathrm{~K})$ and $-4.8 \%$ o $(273.15 \mathrm{~K})$ for HF/3-21G/0.91 (the scaling factor) and B3LYP/6$31 \mathrm{G} / 0.97$ [19]-[21] levels, respectively; and the difference of $\varepsilon_{\max } \mathrm{s}$ between present results and data given by PHVA in Rustad et al. (2008) are $-0.1 \%$ o (=-4.1\%o - $(-4 \%), 298.15 \mathrm{~K})$ and $-3 \%$ o $(=-7 \%$ - $(-4 \%), 298.15 \mathrm{~K})$ for HF/3-21G/0.91 and B3LYP/6-31G/0.97 levels, respectively. For K3866 in Figure 8 and Figure 9, $\varepsilon_{\max } \mathrm{s}$ between present result and data given by Schauble et al. (2006) are $0.015 \%$ o (273.15K) and $-0.031 \%$ (273.15K) for HF/3-21G/0.91 and B3LYP/6-31G/0.97 levels, respectively. It seems clear that K3866 is not sensitive to the exchange-correlation functional/basis set/scaling factor, the number of imaginary frequency $n$ and the magnitude of the frequencies (shown in Table 1 and the "Electronic supplementary materials").

Table 1. Methods/basis_sets/scaling factors ${ }^{1}$ used in Gaussian09 and the results of super-molecules.

\begin{tabular}{|c|c|c|c|c|}
\hline \multirow{2}{*}{ Super-molecule } & \multirow{2}{*}{ Method/Basis_set/Scaling factor } & \multicolumn{3}{|c|}{ Imaginary Frequency $\left(\mathrm{cm}^{-1}\right)^{*}$} \\
\hline & & $n$ & Minimal & Maximal \\
\hline $\mathrm{GeO}(\mathrm{OH})_{3}^{-1}-\left(\mathrm{H}_{2} \mathrm{O}\right)_{30}$ & B3LYP/6-311+G $* 1 * 1.05^{2}$ & 1 & -5.07 & -5.07 \\
\hline $\mathrm{Ge}(\mathrm{OH})_{4}-\left(\mathrm{H}_{2} \mathrm{O}\right)_{30}$ & B3LYP/6-311+G** $/ 1.05^{2}$ & 1 & -22.23 & -22.23 \\
\hline Calcite cluster & $\mathrm{HF} / 3-21 \mathrm{G} / 0.91$ & 76 & -7119.47 & -11.93 \\
\hline Calcite cluster & B3LYP/6-31G/0.97 & 151 & -3321.80 & -69.95 \\
\hline
\end{tabular}

${ }^{1}$ http://cccbdb.nist.gov/. ${ }^{2}$ See Ref. [10]. $n$ is the number of imaginary frequency. ${ }^{*}$ The frequencies correspond to molecules with ${ }^{70} \mathrm{Ge},{ }^{12} \mathrm{C}^{16} \mathrm{O}$.

Table 2. Primitive cell parameter of calcite from CRYSTALL06, with B3LYP/(Ca_86-511d3G, C_6-21Gd, O_8-411d1) ${ }^{1}$.

\begin{tabular}{ccc}
$a=b=c \quad(\AA)$ & $\left.\alpha=\beta=\gamma \quad{ }^{\circ}\right)$ & Volume $\left(\AA^{3}\right)$ \\
\hline 6.47 & 45.90 & 127.64 \\
\hline
\end{tabular}

1http://www.crystal.unito.it.

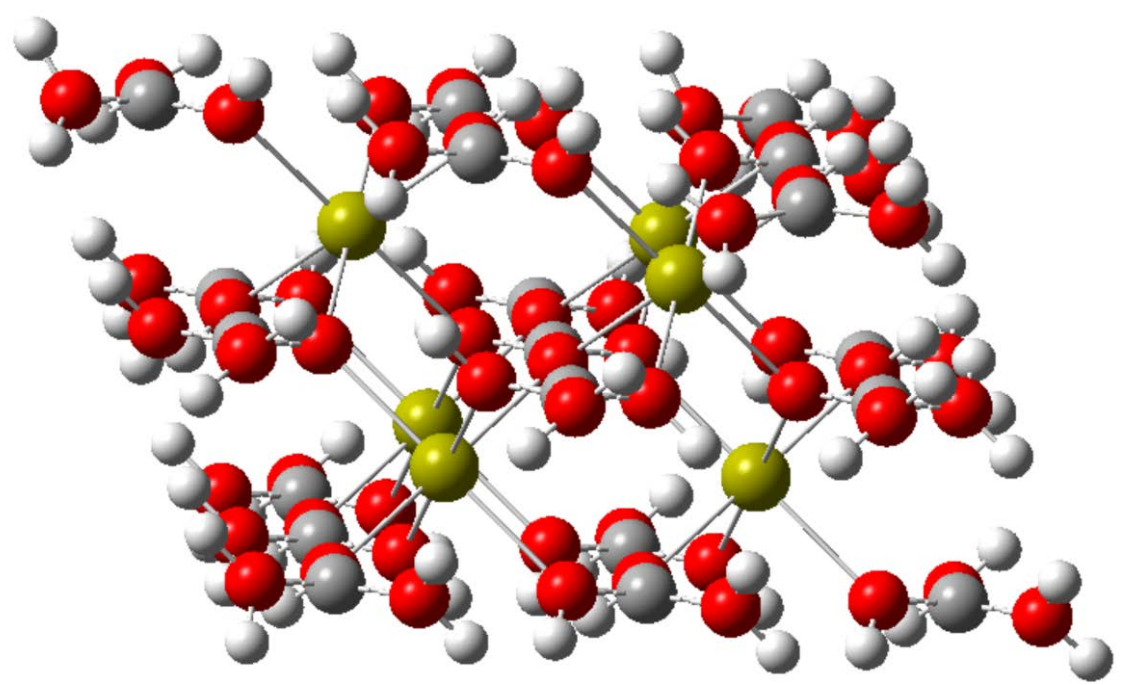

Figure 6. Cluster for calcite (dark gray—carbon, gray—hydrogen, red—oxygen, and yellow—calcium) extracted by the way in Rustad et al. (2008). The length of each $\mathrm{O}-\mathrm{H}$ bond is $0.96 \AA$, and the charge of $\mathrm{H}$ is 0.333 . 


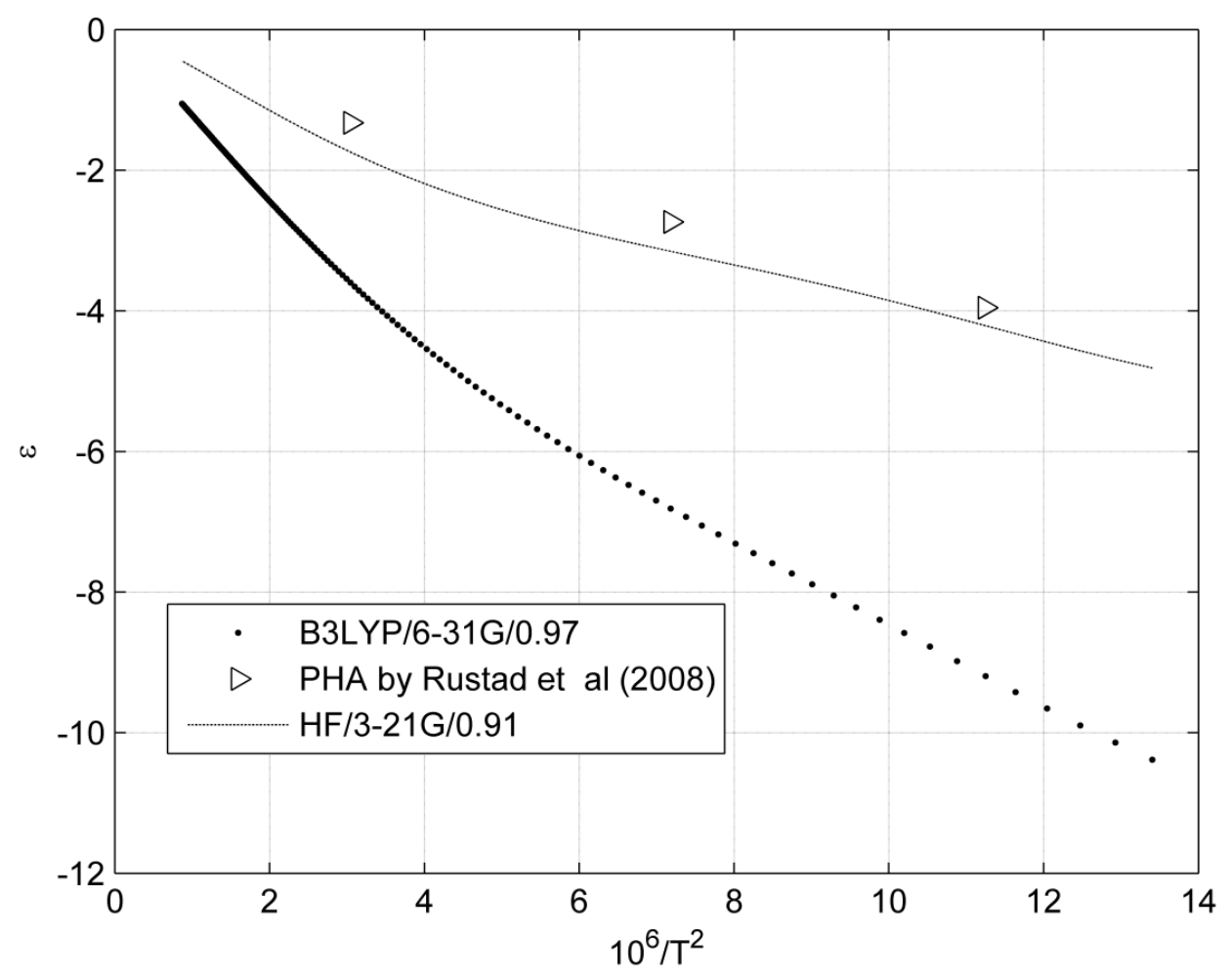

Figure 7. $\varepsilon \alpha_{\mathrm{CaCO}_{3}-\mathrm{C}}$ versus T(K). The reference $\alpha_{\mathrm{CaCO}_{3}-\mathrm{C}} \mathrm{s}$ are from Schauble et al. (2006).

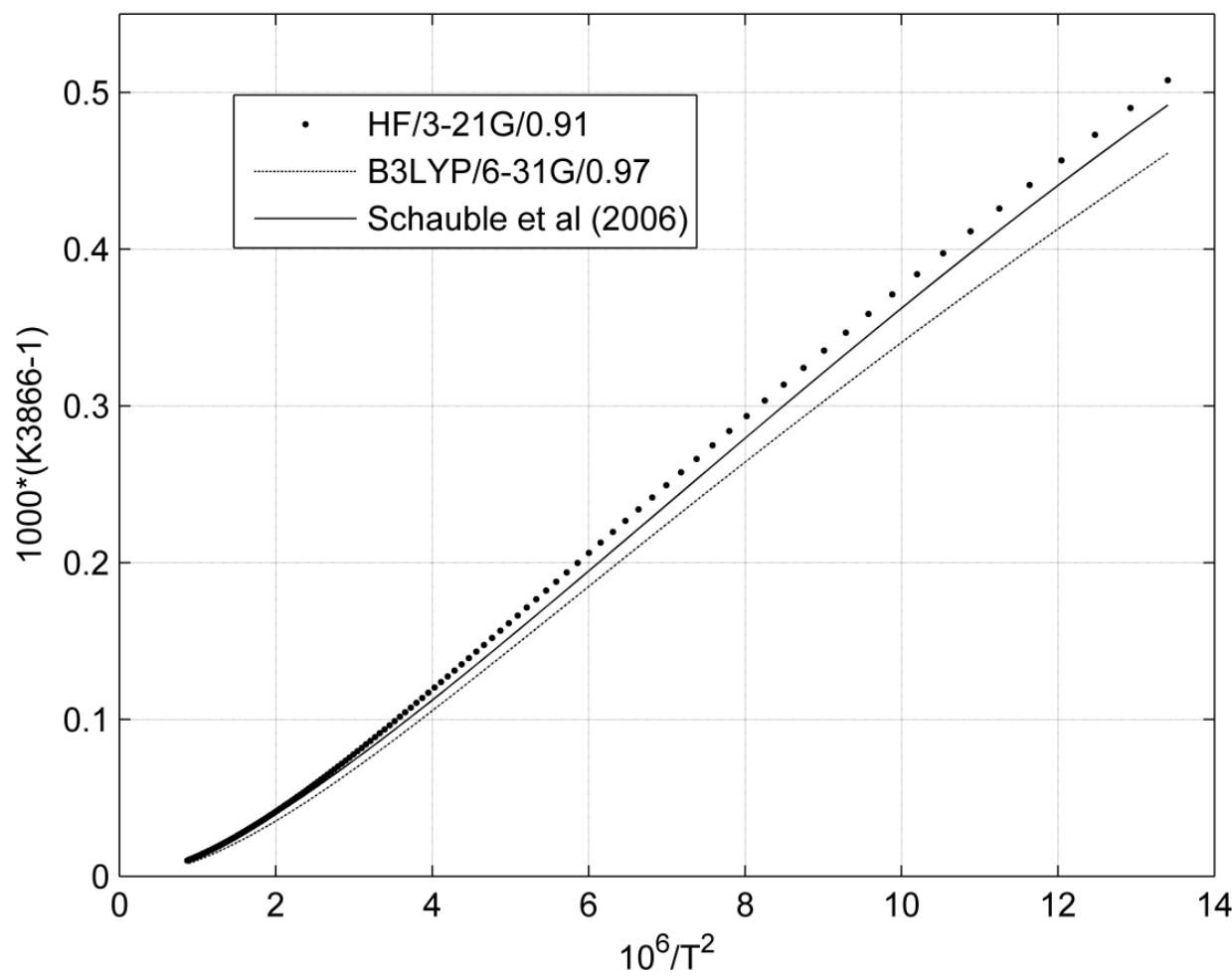

Figure 8. Comparison of K3866s versus T(K). Present K3866s are given by Equation (21) at HF/ 321G/0.91 (dots) and B3LYP/631G/0.97 (solid) levels. Schauble et al.'s (2006) K3866s (bold solid) are given by lattice dynamics. 


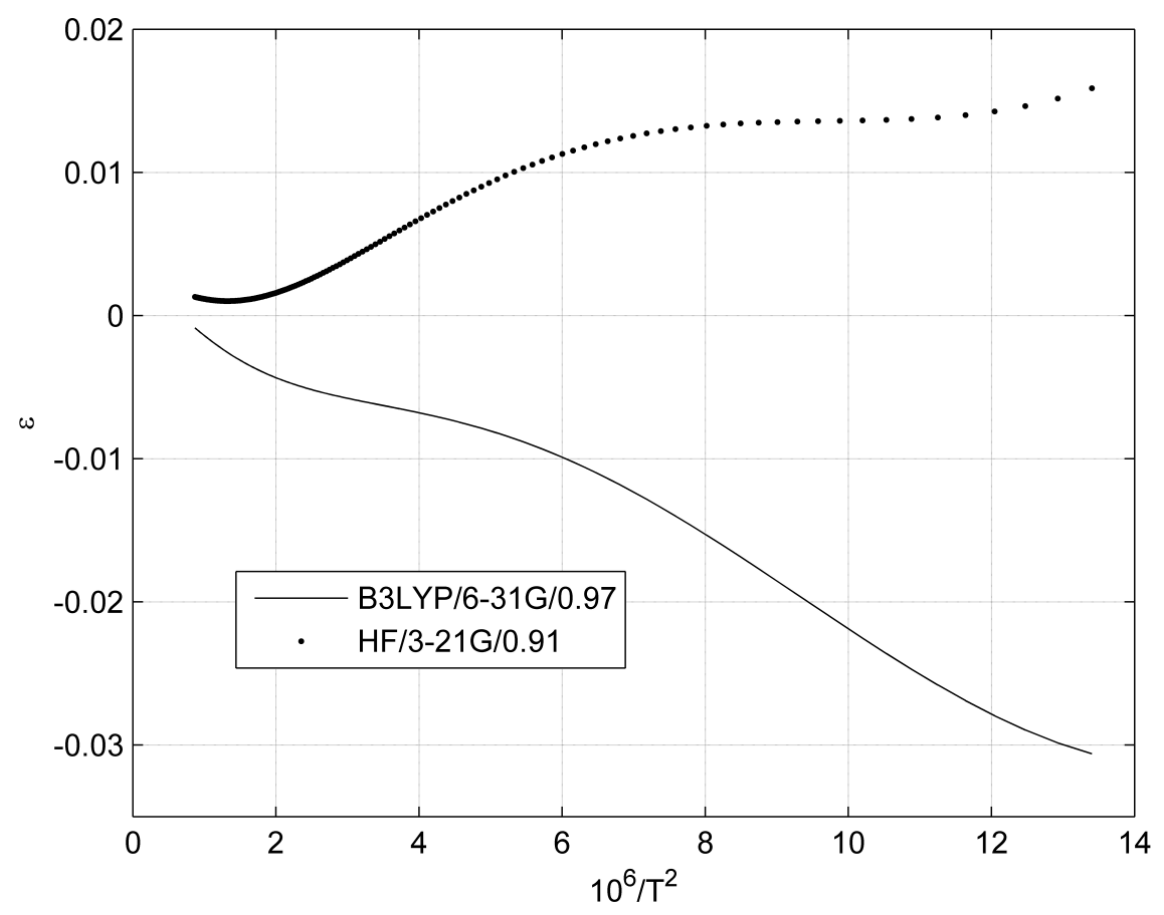

Figure 9. $\varepsilon$ K3866 versus T(K). The reference K3866 is from Schauble et al. (2006).

\section{Conclusion}

For a general cluster for isotope research (defined in Section 2.1), we have a new Equation (21) to calculate the isotope fractionation factor in the cluster. The calculation based on this equation has a clearer background of physical mechanism, which includes the contribution of vibrations of all atoms to the factor, than that based on PHVA. If there is no imaginary frequencies for the cluster, Equation (21) is simplified to be the Urey (1947) or Bigeleisen and Mayer (1947) formula. The examples show that our new algorithm is valid and efficient with high accuracy. Although the accuracy is mathematically high, we again address that present approach should be only used to calculate the isotope fractionation factor.

\section{Acknowledgements}

The author is grateful to Dr. Zhang Zhigang in IGGCAS for helpful discussions. All of the calculations are performed at the IGGCAS computer simulation lab. This work is supported by the National Natural Science Foundation of China (Grant No. 41303047, 41020134003 and 90914010).

\section{References}

[1] Urey, H.C. and Rittenberg, D. (1933) Some Thermodynamic Properties of the $\mathrm{H}^{1} \mathrm{H}^{2}, \mathrm{H}^{2} \mathrm{H}^{2}$ Molecules and Compounds Containing the $\mathrm{H}^{2}$ Atom. Journal of Chemical Physics, 1, 137-143. http://dx.doi.org/10.1063/1.1749265

[2] Urey, H.C. (1947) The Thermodynamic Properties of Isotopic Substances. The Journal of the Chemical Society, 562581. http://pubs.rsc.org/en/Content/ArticleLanding/1947/JR/jr9470000562

[3] Bigeleisen, J. and Mayer, M.G. (1947) Calculation of Equilibrium Constants for Isotopic Exchange Reactions. Journal of Chemical Physics, 15, 261-267. http://dx.doi.org/10.1063/1.1746492

Rustad, J.R., Nelmes, S.L., Jackson, V.E. and Dixon, D.A. (2008) Quantum-Chemical Calculations of Carbon-Isotope Fractionation in $\mathrm{CO}^{2}(\mathrm{~g})$, Aqueous Carbonate Species, and Carbonate Minerals. The Journal of Physical Chemistry A, 112, 542-555. http://dx.doi.org/10.1021/jp076103m http://www.ncbi.nlm.nih.gov/pubmed/18166027

[4] Li, H. and Jensen, J.H (2002) Partial Hessian Vibrational Analysis: The Localization of the Molecular Vibrational Energy and Entropy. Theoretical Chemistry Accounts, 107, 211-219. http://dx.doi.org/10.1007/s00214-001-0317-7

[5] Yuan, J. and Liu, Y. (2011) An Important Method for Calculating Isotope Fractionation in the Solid State Partial Hessian Vibrational Analysis (PHVA). Bulletin of Mineralogy, Petrology and Geochemistry, 30, 472-476. 
[6] Stern, M.J. and Wolfsber, M. (1966) Simplified Procedure for Theoretical Calculation of Isotope Effects Involving Large Molecules. The Journal of Chemical Physics, 45, 4105. http://dx.doi.org/10.1063/1.1727463

[7] Driesner, T., Ha, T.K. and Seward, T.M (2000) Oxygen and Hydrogen Isotope Fractionation by Hydration Complexes of $\mathrm{Li}^{+}, \mathrm{Na}^{+}, \mathrm{K}^{+}, \mathrm{Mg}^{2+}, \mathrm{F}^{-}, \mathrm{Cl}^{-}$, and $\mathrm{Br}^{-}$: A Theoretical Study. Geochimica et Cosmochimica Acta, 64, 3007-3033. http://dx.doi.org/10.1016/S0016-7037(00)00407-5

[8] Liu, Y. and Tossell, J.A. (2005) Ab Initio Molecular Orbital Calculations for Boron Isotope Fractionations on Boric Acids and Borates. Geochimica et Cosmochimica Acta, 69, 3995-4006. http://dx.doi.org/10.1016/j.gca.2005.04.009

[9] Li, X.F., Zhao, H., Tang, M. and Liu, Y. (2009) Theoretical Prediction for Several Important Equilibrium Ge Isotope Fractionation Factors and Geological Implications. Earth and Planetary Science Letters, 287, 1-11. http://dx.doi.org/10.1016/j.epsl.2009.07.027

[10] Pople, J.A., Schlegel, H.B., Krishnan, R., Defrees, D.J., Binkley, J.S., Frisch, M.J., Whiteside, R.A., Hout, R.F. and Hehre, W.J. (1981) Molecular-Orbital Studies of Vibrational Frequencies. International Journal of Quantum Chemistry, 20, 269278. http://dx.doi.org/10.1002/qua.560200829

[11] Frisch, M.J., Trucks, G.W., Schlegel, H.B., Scuseria, G.E., Robb, M.A., Cheeseman, J.R., Montgomery, J.J.A., Vreven, T., Kudin, K.N., Burant, J.C., Millam, J.M., Iyengar, S.S., Tomasi, J., Barone, V., Mennucci, B., Cossi, M., Scalmani, G., Rega, N., Petersson, G.A., Nakatsuji, H., Hada, M., Ehara, M., Toyota, K., Fukuda, R., Hasegawa, J., Ishida, M., Nakajima, T., Honda, Y., Kitao, O., Nakai, H., Klene, M., Li, X., Knox, J.E., Hratchian, H.P., Cross, J.B., Adamo, C., Jaramillo, J., Gomperts, R., Stratmann, R.E., Yazyev, O., Austin, A.J., Cammi, R., Pomelli, C., Ochterski, J.W., Ayala, P.Y., Morokuma, K., Voth, G.A., Salvador, P., Dannenberg, J.J., Zakrzewski, V.G., Dapprich, S., Daniels, A.D., Strain, M.C., Farkas, O., Malick, D.K., Rabuck, A.D., Raghavachari, K., Foresman, J.B., Ortiz, J.V., Cui, Q., Baboul, A.G., Clifford, S., Cioslowski, J., Stefanov, B.B., Liu, G., Liashenko, A., Piskorz, P., Komaromi, I., Martin, R.L., Fox, D.J., Keith, T., Al-Laham, M.A., Peng, C.Y., Nanayakkara, A., Challacombe, M., Gill, P.M.W., Johnson, B., Chen, W., Wong, M.W., Gonzalez, C. and Pople, J.A. (2009) Gaussian 09, Revision A.01. Gaussian, Inc., Wallingford.

[12] Fong, C.F.C.M., Kee, D.D. and Kaloni, P.N. (2002) Advanced Mathematics for Engineering and Science. World Scientific Publishing Co. Pte. Ltd., Singapore.

[13] Born, M. and Oppenheimer, R. (1927) On the Quantum Theory of Molecules. Annalen der Physik, 84, 457-484. http://dx.doi.org/10.1002/andp.19273892002

[14] Levine, I.N. (1995) Physical Chemistry. 4th Edition, McGraw-Hill, Inc., New York.

[15] Wilson, E.B.J., Decius, J.C. and Cross, P.C. (1955) Molecular Vibrations: The Theory of Infrared and Raman Spectra. Dover Publications, New York.

[16] Dovesi, R., Saunders, V.R., Roetti, C., Orlando, R., Zicovich-Wilson, C.M., Pascale, F., Civalleri, B., Doll, K., Harrison, N.M., Bush, I.J., D’Arco, P. and Llunell, M. (2006) CRYSTAL06 User’s Manual. University of Torino, Torino.

[17] Schauble, E.A., Ghosh, P. and Eiler, J.M. (2006) Preferential Formation of ${ }^{13} \mathrm{C}-{ }^{18} \mathrm{O}$ Bonds in Carbonate Minerals, Estimated Using First-Principles Lattice Dynamics. Geochimica et Cosmochimica Acta, 70, 2510-2529. http://dx.doi.org/10.1016/j.gca.2006.02.011

[18] Becke, A.D. (1993) Density-Functional Thermochemistry. III. The Role of Exact Exchange. Journal of Chemical Physics, 98, 5648-5652. http://dx.doi.org/10.1063/1.464913

[19] Lee, C.T., Yang, W.T. and Parr, R.G. (1988) Development of the Colle-Salvetti Correlation-Energy Formula into a Functional of the Electron-Density. Physical Review B, 37, 785-789. http://dx.doi.org/10.1103/PhysRevB.37.785

[20] Vosko, S.H., Wilk, L. and Nusair, M. (1980) Accurate Spin-Dependent Electron Liquid Correlation Energies for Local Spin-Density Calculations-A Critical Analysis. Canadian Journal of Physics, 58, 1200-1211. http://dx.doi.org/10.1139/p80-159

[21] Stephens, P.J., Devlin, F.J., Chabalowski, C.F. and Frisch, M.J. (1994) Ab Initio Calculation of Vibrational Absorption and Circular-Dichroism Spectra Using Density-Functional Force-Fields. Journal of Physical Chemistry, 98, 1162311627. http://dx.doi.org/10.1021/j100096a001 


\section{Electronic Supplementary Materials}

Optimized Geometries and Frequencies $\left(\mathrm{cm}^{-1}\right)$ of All Clusters in the Text

1. $\mathrm{GeO}(\mathrm{OH})_{3}^{-}-\left(\mathrm{H}_{2} \mathrm{O}\right)_{30}$, Optimized Geometry (B3LYP/6-311+G(d,p))

\begin{tabular}{|c|c|c|c|}
\hline 32 & -0.596615 & -0.016176 & 0.000428 \\
\hline 8 & -1.344083 & -1.535373 & -0.256154 \\
\hline 8 & 0.759944 & 0.495686 & -1.052984 \\
\hline 1 & 0.443163 & 0.681858 & -1.974307 \\
\hline 8 & -1.786742 & 1.350206 & -0.118265 \\
\hline 1 & -2.720088 & 1.028346 & -0.088157 \\
\hline 8 & 0.191339 & 0.087024 & 1.623802 \\
\hline 1 & 0.004685 & -0.737764 & 2.138406 \\
\hline 8 & -0.250691 & -2.287403 & 2.973304 \\
\hline 1 & 0.019361 & -2.930021 & 2.282848 \\
\hline 1 & -1.228588 & -2.307321 & 2.948463 \\
\hline 8 & 2.953986 & -0.371657 & 1.902444 \\
\hline 1 & 2.050203 & -0.124577 & 1.629309 \\
\hline 1 & 3.137374 & -1.223120 & 1.446393 \\
\hline 8 & 0.060105 & 4.615808 & -0.759392 \\
\hline 1 & 0.739234 & 3.964630 & -0.477242 \\
\hline 1 & 0.599742 & 5.282466 & -1.211689 \\
\hline 8 & -0.289293 & 0.876900 & -3.531483 \\
\hline 1 & -0.901161 & 0.113044 & -3.510001 \\
\hline 1 & -0.824485 & 1.685431 & -3.374504 \\
\hline 8 & -1.755047 & 3.706872 & 1.253116 \\
\hline 1 & -1.690600 & 2.869490 & 0.738934 \\
\hline 1 & -1.265590 & 4.352361 & 0.712271 \\
\hline 8 & 3.389510 & 2.367456 & 2.131182 \\
\hline 1 & 3.576766 & 1.417751 & 2.025324 \\
\hline 1 & 2.924444 & 2.401424 & 2.990733 \\
\hline 8 & -1.670071 & 3.015607 & -2.456210 \\
\hline 1 & -1.074243 & 3.692920 & -2.086833 \\
\hline 1 & -1.856875 & 2.457840 & -1.680515 \\
\hline 8 & 2.162197 & 2.813700 & -0.259564 \\
\hline 1 & 2.575669 & 2.715749 & 0.637460 \\
\hline 1 & 1.738555 & 1.956790 & -0.475235 \\
\hline 8 & -4.377005 & 2.993431 & 1.696156 \\
\hline 1 & -3.523570 & 3.461707 & 1.550722 \\
\hline 1 & -4.983659 & 3.636559 & 2.074156 \\
\hline 8 & -3.181781 & 0.706008 & 3.265501 \\
\hline 1 & -2.343752 & 1.159156 & 3.480253 \\
\hline 1 & -3.719911 & 1.386249 & 2.832853 \\
\hline 8 & -2.911860 & -1.838524 & 2.088474 \\
\hline 1 & -2.443170 & -1.743903 & 1.226000 \\
\hline 1 & -3.001797 & -0.937950 & 2.469805 \\
\hline 8 & -0.658478 & 2.071125 & 3.370753 \\
\hline 1 & -0.302726 & 1.433256 & 2.715372 \\
\hline 1 & -0.905982 & 2.848861 & 2.839829 \\
\hline 8 & 2.137763 & -1.312646 & 4.387196 \\
\hline 1 & 1.247248 & -1.616254 & 4.140448 \\
\hline 1 & 2.495543 & -0.920032 & 3.560275 \\
\hline 8 & 0.393874 & -3.491482 & 0.549170 \\
\hline
\end{tabular}




$\begin{array}{llll}1 & 0.284286 & -4.281461 & -0.021179 \\ 1 & -0.227554 & -2.821388 & 0.163978 \\ 8 & -1.940404 & -1.379942 & -2.887707 \\ 1 & -1.782683 & -1.515882 & -1.912623 \\ 1 & -2.859030 & -1.073731 & -2.957104 \\ 8 & 1.934450 & 1.952057 & 4.580577 \\ 1 & 2.013396 & 1.014557 & 4.808848 \\ 1 & 0.997211 & 2.080198 & 4.346005 \\ 8 & 2.685883 & 5.221302 & -1.833844 \\ 1 & 3.157977 & 5.016948 & -2.647923 \\ 1 & 2.762427 & 4.407197 & -1.310465 \\ 8 & -4.483698 & 0.827015 & -0.170051 \\ 1 & -4.866731 & 0.023592 & 0.283481 \\ 1 & -4.729783 & 1.607381 & 0.356255 \\ 8 & 3.523484 & -3.658566 & 3.670302 \\ 1 & 3.192271 & -4.436502 & 4.127411 \\ 1 & 3.082582 & -2.889803 & 4.092676 \\ 8 & 3.144140 & -2.957518 & 0.949211 \\ 1 & 2.213532 & -3.187023 & 0.738114 \\ 1 & 3.337150 & -3.377177 & 1.810134 \\ 8 & 4.597973 & -2.272653 & -1.443332 \\ 1 & 4.249163 & -2.608847 & -0.599296 \\ 1 & 3.805391 & -2.173335 & -2.005481 \\ 8 & 3.971820 & 2.066121 & -2.562518 \\ 1 & 3.474098 & 2.275912 & -1.757844 \\ 1 & 4.672941 & 1.448873 & -2.261045 \\ 8 & -4.622492 & -0.191863 & -2.707139 \\ 1 & -4.613120 & 0.336281 & -1.875300 \\ 1 & -4.945259 & 0.393223 & -3.399227 \\ 8 & 0.094938 & -5.351034 & -1.479023 \\ 1 & 0.066776 & -4.701081 & -2.224952 \\ 1 & -0.605132 & -5.985306 & -1.651906 \\ 8 & 2.154240 & -1.645105 & -2.764170 \\ 1 & 1.787052 & -1.130723 & -2.027964 \\ 1 & 2.341278 & -0.952721 & -3.448065 \\ 8 & 0.062239 & -3.445681 & -3.426849 \\ 1 & -0.699771 & -2.843083 & -3.412012 \\ 1 & 0.839447 & -2.866540 & -3.286664 \\ 8 & -5.565204 & -2.680908 & -1.715460 \\ 1 & -5.326260 & -1.869024 & -2.200186 \\ 1 & -4.867932 & -3.311400 & -1.922399 \\ 8 & 5.794520 & 0.199586 & -1.553250 \\ 1 & 6.163317 & 0.385340 & -0.685046 \\ 1 & 5.406249 & -0.706661 & -1.488260 \\ 8 & -5.418074 & -1.452748 & 0.853642 \\ 1 & -5.538109 & -2.031146 & 0.077259 \\ 1 & -4.657728 & -1.812485 & 1.353188 \\ 8 & 2.453482 & 0.523722 & -4.378814 \\ 1 & 2.977952 & 1.141617 & -3.826756 \\ 1 & 1.532754 & 0.824715 & -4.319686 \\ & & \end{array}$

Frequencies (not scaled) Ge-70:

$-5.0668$

25.2219

26.4774

29.6821

31.2812

36.9882 


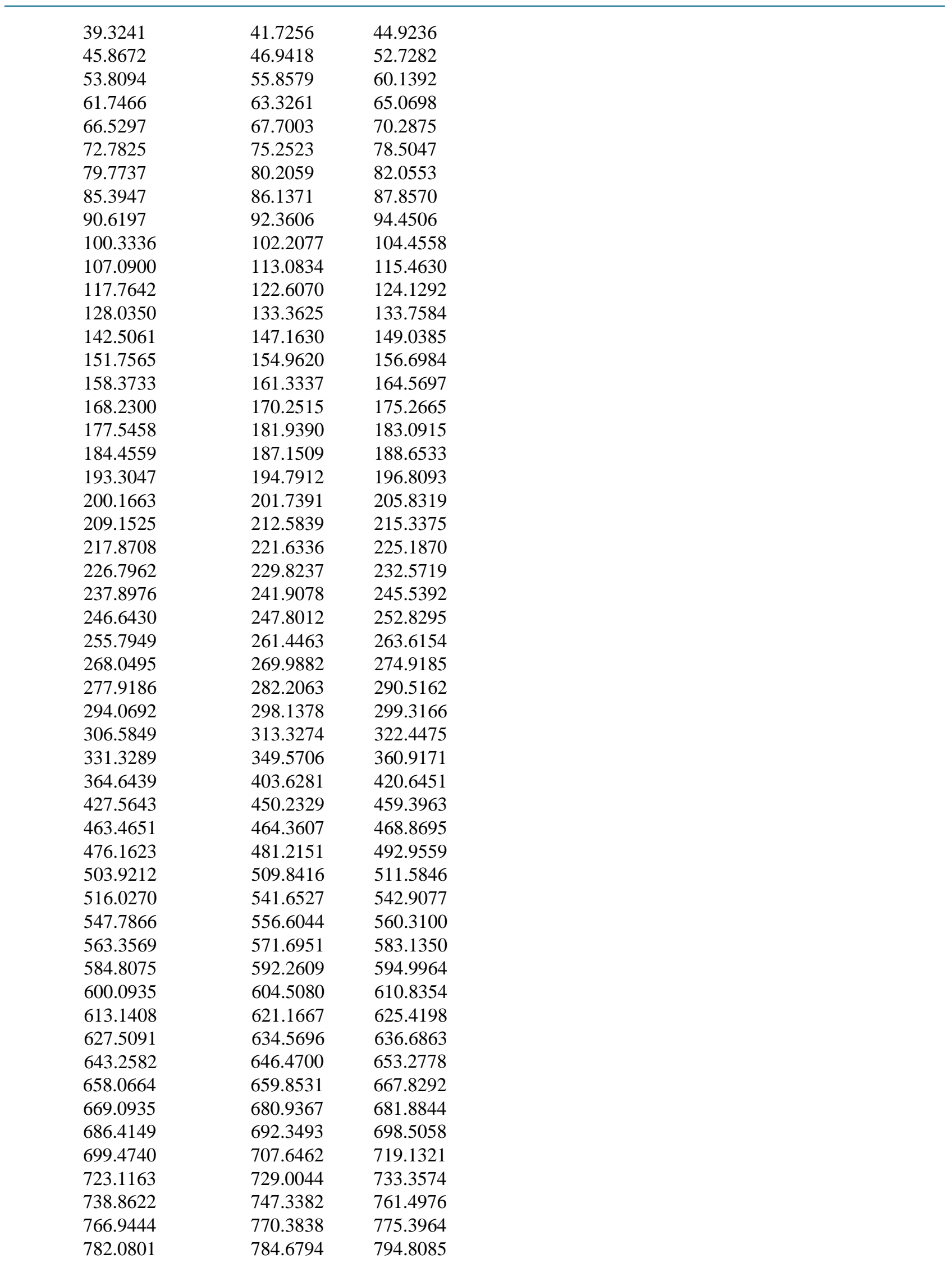




$\begin{array}{lcl}807.3475 & 812.2181 & 816.9983 \\ 821.4843 & 836.5895 & 840.7348 \\ 854.0293 & 855.9679 & 863.1113 \\ 871.5906 & 881.6800 & 892.9068 \\ 908.1346 & 911.2110 & 919.8440 \\ 921.6876 & 929.5758 & 935.3924 \\ 953.3906 & 982.5497 & 1005.9739 \\ 1010.4208 & 1023.2002 & 1033.2698 \\ 1035.8893 & 1059.6119 & 1096.4750 \\ 1217.8723 & 1243.5883 & 1252.8465 \\ 1647.3773 & 1649.8385 & 1654.6022 \\ 1664.2926 & 1666.0608 & 1670.3453 \\ 1672.3593 & 1673.5404 & 1681.0771 \\ 1683.2199 & 1684.8552 & 1688.1825 \\ 1690.5686 & 1693.9056 & 1697.0781 \\ 1700.2305 & 1702.3239 & 1703.2890 \\ 1704.0138 & 1705.4095 & 1707.3200 \\ 1710.0453 & 1713.1124 & 1719.9819 \\ 1722.3737 & 1725.2227 & 1727.4139 \\ 1734.4425 & 1738.0789 & 1740.3854 \\ 3139.4735 & 3173.1036 & 3264.0573 \\ 3276.2085 & 3279.2707 & 3307.9602 \\ 3312.4005 & 3332.1576 & 3350.8184 \\ 3366.9917 & 3393.3387 & 3405.0437 \\ 3409.5885 & 3449.8980 & 3463.4164 \\ 3466.6185 & 3473.8176 & 3476.9342 \\ 3485.1213 & 3496.5444 & 3498.2129 \\ 3505.5025 & 3509.8664 & 3515.0034 \\ 3530.9420 & 3544.1369 & 3546.2703 \\ 3548.3123 & 3561.4907 & 3562.9984 \\ 3580.5408 & 3588.5519 & 3596.5550 \\ 3598.1483 & 3606.7150 & 3608.6345 \\ 3611.0240 & 3631.4225 & 3639.9418 \\ 3644.3322 & 3655.3541 & 3669.7740 \\ 3675.5280 & 3677.9754 & 3682.0254 \\ 3685.9738 & 3695.0533 & 3695.5546 \\ 3699.0678 & 3720.0607 & 3724.2312 \\ 3736.9712 & 3743.2078 & 3748.3595 \\ 3764.0547 & 3778.6409 & 3871.2555 \\ 3873.3062 & 3880.5070 & 3882.0263 \\ 3885.7924 & 3888.5458 & 3895.7477 \\ \text { Frequencies } \text { (not scaled) Ge-74: } & \\ -5.0643 & 25.2133 & 26.4674 \\ 29.6708 & 31.2793 & 36.9845 \\ 39.2968 & 41.6789 & 44.9075 \\ 45.8075 & 46.9055 & 52.7095 \\ 53.7415 & 55.7294 & 60.1157 \\ 61.7143 & 63.2936 & 64.9343 \\ 66.5190 & 67.6923 & 70.2713 \\ 79.7200 & 80.0094 & 81.9800 \\ 85.3455 & 86.1141 & 87.8169 \\ 90.6059 & 92.3203 & 94.3766 \\ 100.3308 & 102.1357 & 104.4006\end{array}$




$\begin{array}{lll}107.0656 & 113.0744 & 115.4538 \\ 117.6493 & 122.5855 & 124.1085 \\ 127.9737 & 133.3224 & 133.7436 \\ 142.4367 & 147.0887 & 148.8962 \\ 151.5739 & 154.9430 & 156.6208 \\ 158.3356 & 161.1584 & 164.5454 \\ 168.1628 & 170.1113 & 175.1637 \\ 176.8799 & 181.8885 & 182.9734 \\ 184.3915 & 187.1507 & 188.6341 \\ 193.2785 & 194.7849 & 196.7696 \\ 200.1267 & 201.5844 & 205.7502 \\ 208.7339 & 212.4106 & 215.2986 \\ 217.8011 & 221.5395 & 225.1432 \\ 226.6914 & 229.7992 & 232.5563 \\ 237.7252 & 241.8830 & 245.5309 \\ 246.6399 & 247.7760 & 252.8223 \\ 255.6675 & 261.4198 & 263.5830 \\ 267.9678 & 269.9575 & 274.7377 \\ 277.8046 & 282.2005 & 290.5083 \\ 293.9856 & 298.0922 & 299.2603 \\ 306.4116 & 313.2903 & 322.0383 \\ 330.7451 & 348.8647 & 360.6636 \\ 364.1913 & 403.6273 & 420.6422 \\ 427.5643 & 450.2132 & 459.3936 \\ 463.4534 & 464.3587 & 468.8661 \\ 476.1612 & 481.2143 & 492.9507 \\ 503.9182 & 509.8331 & 511.5522 \\ 516.0053 & 541.6493 & 542.9005 \\ 547.7711 & 556.5982 & 560.3098 \\ 563.3369 & 571.6622 & 583.1215 \\ 584.7932 & 592.1727 & 594.9515 \\ 600.0891 & 604.4338 & 610.7464 \\ 612.8143 & 621.0248 & 625.2868 \\ 627.3615 & 634.2759 & 634.9881 \\ 642.8499 & 646.1902 & 652.9881 \\ 657.9451 & 659.5618 & 666.9272 \\ 668.8740 & 679.2008 & 681.5888 \\ 686.2180 & 692.3272 & 698.3964 \\ 699.4308 & 707.6221 & 719.0922 \\ 722.9846 & 728.9587 & 733.3143 \\ 738.7843 & 747.3133 & 761.4888 \\ 766.9324 & 770.3400 & 775.3799 \\ 781.9760 & 784.6409 & 794.7308 \\ 804.4872 & 812.1692 & 816.8852 \\ 820.7164 & 836.5632 & 840.7292 \\ 854.0113 & 855.9532 & 863.0288 \\ 871.5387 & 881.6729 & 892.8805 \\ 908.1313 & 911.1394 & 919.8339 \\ 921.6810 & 929.5656 & 935.3104 \\ 953.3631 & 982.5350 & 1005.9565 \\ 1010.4001 & 1023.1979 & 1033.2637 \\ 1035.8835 & 1059.6006 & 1096.4746 \\ 1217.7390 & 1243.5246 & 1252.7315\end{array}$




$\begin{array}{lll}1647.3771 & 1649.8385 & 1654.6021 \\ 1664.2922 & 1666.0583 & 1670.3450 \\ 1672.3591 & 1673.5403 & 1681.0762 \\ 1683.2195 & 1684.8550 & 1688.1822 \\ 1690.5684 & 1693.9055 & 1697.0776 \\ 1700.2301 & 1702.3236 & 1703.2876 \\ 1704.0127 & 1705.4093 & 1707.3197 \\ 1710.0450 & 1713.1124 & 1719.9809 \\ 1722.3735 & 1725.2223 & 1727.4132 \\ 1734.4419 & 1738.0774 & 1740.3852 \\ 3139.4729 & 3173.0991 & 3264.0540 \\ 3276.2045 & 3279.2696 & 3307.9592 \\ 3312.4002 & 3332.1575 & 3350.8181 \\ 3366.9916 & 3393.3342 & 3405.0437 \\ 3409.5862 & 3449.8969 & 3463.4157 \\ 3466.6183 & 3473.8166 & 3476.9331 \\ 3485.1202 & 3496.5443 & 3498.2128 \\ 3505.5018 & 3509.8663 & 3515.0034 \\ 3530.9411 & 3544.1367 & 3546.2701 \\ 3548.3123 & 3561.4907 & 3562.9981 \\ 3580.5407 & 3588.5518 & 3596.5547 \\ 3598.1481 & 3606.7150 & 3608.6331 \\ 3611.0237 & 3631.4224 & 3639.9416 \\ 3644.3322 & 3655.3538 & 3669.7739 \\ 3675.5276 & 3677.9754 & 3682.0254 \\ 3685.9734 & 3695.0532 & 3695.5546 \\ 3699.0677 & 3720.0605 & 3724.2312 \\ 3736.9709 & 3743.2077 & 3748.3594 \\ 3764.0546 & 3778.6409 & 3871.2555 \\ 3873.3062 & 3880.5070 & 3882.0263 \\ 3885.7924 & 3888.5458 & 3895.7477\end{array}$

2. $\mathrm{Ge}(\mathrm{OH})_{4}-\left(\mathrm{H}_{2} \mathrm{O}\right)_{30}$, Optimized Geometry (B3LYP/6-311+G(d,p))

$\begin{array}{llll}32 & -0.169708 & 0.490256 & -0.101782 \\ 8 & -0.891759 & -1.088721 & 0.147186 \\ 1 & -0.372608 & -1.885699 & -0.162666 \\ 8 & 1.563547 & 0.206411 & -0.387786 \\ 1 & 2.063147 & 0.960311 & -0.808992 \\ 8 & -0.843853 & 1.361120 & -1.507174 \\ 1 & -1.554320 & 2.028714 & -1.273462 \\ 8 & -0.489885 & 1.547516 & 1.283691 \\ 1 & 0.015041 & 1.305742 & 2.137938 \\ 8 & 0.308410 & -3.380269 & -0.467372 \\ 1 & 1.219966 & -3.308631 & -0.801008 \\ 1 & -0.264685 & -3.749271 & -1.197788 \\ 8 & 0.843576 & 0.998221 & 3.424687 \\ 1 & 1.658254 & 0.535809 & 3.134690 \\ 1 & 0.336770 & 0.344851 & 3.969448 \\ 8 & 2.981069 & -0.077766 & 2.059751 \\ 1 & 3.151125 & -1.039648 & 2.230560 \\ 1 & 2.488563 & -0.081917 & 1.218333 \\ 8 & 3.161997 & 2.022119 & -1.466740\end{array}$




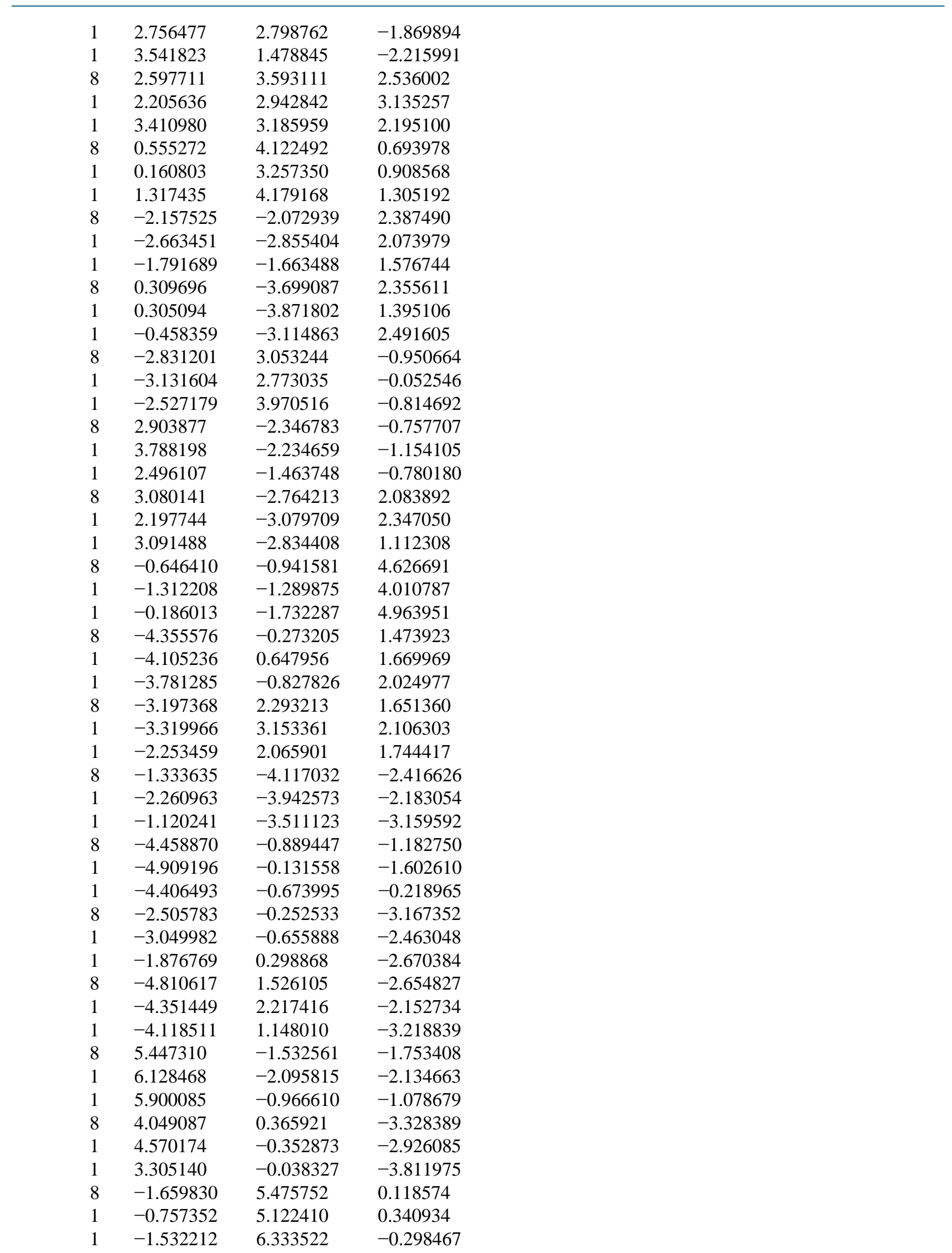




$\begin{array}{llll}8 & 4.647910 & 1.885172 & 1.028067 \\ 1 & 4.148960 & 1.160779 & 1.463045 \\ 1 & 4.175263 & 2.067773 & 0.195242 \\ 8 & 6.594152 & 0.156687 & 0.039851 \\ 1 & 7.179097 & -0.109557 & 0.755298 \\ 1 & 6.005328 & 0.852023 & 0.412059 \\ 8 & -4.064501 & -3.553669 & -1.440730 \\ 1 & -4.805431 & -4.007242 & -1.856060 \\ 1 & -4.310997 & -2.596506 & -1.416687 \\ 8 & 1.621749 & -0.315308 & -4.666636 \\ 1 & 1.695099 & -0.327285 & -5.627085 \\ 1 & 1.243127 & 0.561545 & -4.434560 \\ 8 & -0.766575 & -2.156433 & -4.306221 \\ 1 & 0.083617 & -1.693766 & -4.357868 \\ 1 & -1.416104 & -1.476753 & -4.038728 \\ 8 & -3.433825 & -4.229589 & 1.223869 \\ 1 & -2.992468 & -5.084296 & 1.224070 \\ 1 & -3.690367 & -4.054711 & 0.295513 \\ 8 & 0.684981 & 2.104095 & -3.701568 \\ 1 & 0.195863 & 2.743542 & -4.228966 \\ 1 & 0.125078 & 1.910628 & -2.920334 \\ 8 & -3.260165 & 4.939510 & 2.377751 \\ 1 & -2.708552 & 5.307102 & 1.660372 \\ 1 & -3.014833 & 5.399151 & 3.185657 \\ 8 & 0.654477 & -3.424814 & 5.128548 \\ 1 & 0.467173 & -4.116740 & 5.768920 \\ 1 & 0.666824 & -3.849176 & 4.254563\end{array}$

Frequencies (not scaled) Ge-70:

$\begin{array}{lll}-22.2257 & 19.6523 & 28.3783 \\ 33.0113 & 33.5203 & 35.2689 \\ 37.7866 & 44.6476 & 44.9917 \\ 46.0637 & 48.5373 & 49.8224 \\ 50.8145 & 53.3679 & 55.7730 \\ 56.8876 & 59.0893 & 60.5302 \\ 61.0407 & 64.9039 & 67.2880 \\ 69.3018 & 69.6522 & 70.4868 \\ 73.5490 & 75.2931 & 76.7631 \\ 77.1337 & 80.3302 & 81.3045 \\ 84.0066 & 87.7519 & 90.8378 \\ 92.1415 & 94.6306 & 104.2453 \\ 106.0577 & 108.7853 & 109.2310 \\ 111.6486 & 115.2207 & 115.9120 \\ 120.4108 & 124.2837 & 125.5232 \\ 135.3280 & 139.2283 & 144.0345 \\ 144.6376 & 149.4271 & 153.5566 \\ 156.2225 & 158.3019 & 158.8944 \\ 162.4860 & 165.5643 & 168.5052 \\ 172.6976 & 173.7904 & 178.2958 \\ 178.4836 & 181.8807 & 186.2633 \\ 190.2791 & 192.8613 & 196.3889 \\ 198.7425 & 202.0265 & 204.6728 \\ 207.8245 & 213.7474 & 214.0551 \\ 218.7214 & 219.5876 & 226.0944\end{array}$




$\begin{array}{lll} & & \\ 231.1274 & 235.5255 & 242.1860 \\ 244.5260 & 248.6356 & 254.5778 \\ 256.3999 & 256.7121 & 258.7711 \\ 266.7019 & 269.7869 & 278.6038 \\ 281.6766 & 286.6844 & 289.6794 \\ 289.9550 & 308.0023 & 311.6381 \\ 315.1413 & 318.5303 & 323.3357 \\ 328.2466 & 328.7730 & 338.7565 \\ 342.2524 & 346.9835 & 349.1994 \\ 354.0360 & 361.1901 & 374.1939 \\ 385.5776 & 412.1751 & 421.1706 \\ 427.6115 & 438.1148 & 439.4553 \\ 447.6792 & 451.3011 & 467.0777 \\ 473.4897 & 477.8566 & 479.7783 \\ 482.3430 & 497.1452 & 508.0102 \\ 512.1131 & 522.5043 & 527.1514 \\ 529.5171 & 535.5315 & 537.1088 \\ 545.0029 & 546.8211 & 551.9551 \\ 559.4088 & 566.4328 & 571.6828 \\ 576.8908 & 584.8178 & 591.8511 \\ 594.7960 & 598.8018 & 609.7641 \\ 629.8630 & 630.7873 & 641.8239 \\ 648.4057 & 658.9653 & 665.6999 \\ 672.4113 & 676.6044 & 679.5191 \\ 684.5625 & 687.2885 & 690.8769 \\ 703.8074 & 708.1784 & 708.8463 \\ 714.7296 & 723.0098 & 724.6398 \\ 729.1566 & 736.3073 & 740.1334 \\ 747.0604 & 750.1703 & 751.9887 \\ 761.3937 & 762.2208 & 770.3300 \\ 772.0863 & 777.9889 & 781.4050 \\ 792.8682 & 798.1769 & 802.4982 \\ 813.1956 & 827.7274 & 833.9391 \\ 853.4112 & 861.4767 & 862.9729 \\ 870.8832 & 889.5086 & 910.0778 \\ 916.2186 & 919.5493 & 923.8791 \\ 934.5573 & 942.7036 & 946.6920 \\ 961.6640 & 968.9220 & 1026.8224 \\ 1032.2364 & 1058.7412 & 1070.9684 \\ 1162.9268 & 1264.8927 & 1273.6644 \\ 1293.0930 & 1347.7565 & 1623.7113 \\ 1631.9879 & 1654.9239 & 1655.5733 \\ 1656.4717 & 1664.4790 & 1666.6971 \\ 1667.3911 & 1668.4594 & 1669.5516 \\ 1671.9284 & 1676.5365 & 1677.1699 \\ 1679.1071 & 1684.7503 & 1688.8095 \\ 1691.5839 & 1692.8089 & 1696.3112 \\ 1697.7049 & 1703.1011 & 1703.7148 \\ 1708.9120 & 1716.4307 & 1720.9780 \\ 1724.9416 & 1725.8472 & 1734.4971 \\ 1735.7154 & 1738.6210 & 2731.2509 \\ 3066.2107 & 3074.8076 & 3100.3878 \\ 3199.0762 & 3215.3294 & 3249.9866\end{array}$




$\begin{array}{lrl}3301.5269 & 3318.1188 & 3329.5014 \\ 3350.0309 & 3377.9426 & 3391.6679 \\ 3436.8102 & 3454.5668 & 3461.0578 \\ 3483.2468 & 3493.2054 & 3516.6984 \\ 3524.6411 & 3525.8713 & 3533.1320 \\ 3559.0827 & 3561.4088 & 3573.0608 \\ 3585.5851 & 3585.8865 & 3587.3008 \\ 3593.4910 & 3601.2218 & 3609.0311 \\ 3618.0837 & 3624.1737 & 3625.5726 \\ 3630.7311 & 3631.9500 & 3639.1683 \\ 3646.9939 & 3650.3645 & 3653.2042 \\ 3655.8800 & 3668.5314 & 3670.0370 \\ 3683.3482 & 3692.1045 & 3693.8278 \\ 3694.6933 & 3707.5970 & 3708.1947 \\ 3711.3797 & 3741.2914 & 3745.0075 \\ 3773.4150 & 3806.2244 & 3847.1549 \\ 3861.3956 & 3866.8538 & 3871.4351 \\ 3873.8987 & 3874.6260 & 3881.0434 \\ 3882.3324 & 3890.9624 & 3896.6846 \\ \text { Frequencies (not scaled) Ge-74: } & \\ -22.2237 & 19.6322 & 28.3402 \\ 32.9965 & 33.4966 & 35.2481 \\ 37.7513 & 44.6305 & 44.9851 \\ 46.0075 & 48.5024 & 49.7140 \\ 50.7903 & 53.3426 & 55.7118 \\ 56.8450 & 59.0707 & 60.4978 \\ 60.9914 & 64.8902 & 67.1390 \\ 69.2678 & 69.6257 & 70.4493 \\ 73.5027 & 75.2538 & 76.7376 \\ 77.1179 & 80.3198 & 81.2788 \\ 83.9661 & 87.7067 & 90.8316 \\ 92.1249 & 94.6054 & 104.1907 \\ 106.0269 & 108.7266 & 109.1997 \\ 111.6414 & 115.1702 & 115.8895 \\ 120.3945 & 124.2661 & 125.3959 \\ 135.3008 & 139.1899 & 143.8901 \\ 144.5662 & 149.3522 & 153.4793 \\ 156.0744 & 158.2597 & 158.8255 \\ 162.2922 & 165.4776 & 168.1795 \\ 172.5521 & 173.7275 & 178.2323 \\ 178.4647 & 181.7611 & 186.0975 \\ 190.2630 & 192.7086 & 196.3613 \\ 198.6994 & 201.9084 & 204.4793 \\ 207.6189 & 213.5306 & 214.0274 \\ 218.6539 & 219.5302 & 226.0359 \\ 230.7868 & 235.5030 & 242.0604 \\ 244.5111 & 248.6188 & 254.5094 \\ 256.3645 & 256.6485 & 258.6888 \\ 266.6742 & 269.6732 & 278.4016 \\ 281.6238 & 286.6697 & 288.9836 \\ 289.9138 & 307.7967 & 311.6085 \\ 314.9870 & 318.5170 & 323.2948 \\ 328.1536 & 328.7159 & 338.7140\end{array}$




$\begin{array}{lll} & & \\ 342.2270 & 346.6134 & 349.1069 \\ 353.7036 & 361.1077 & 373.9345 \\ 385.5225 & 412.0475 & 421.1137 \\ 427.6095 & 438.1024 & 439.4527 \\ 447.6759 & 451.2947 & 467.0742 \\ 473.4884 & 477.8425 & 479.7754 \\ 482.3408 & 497.1383 & 508.0059 \\ 512.0945 & 522.4937 & 527.1491 \\ 529.5158 & 535.5285 & 537.0927 \\ 545.0008 & 546.8127 & 551.9513 \\ 559.3948 & 566.4112 & 571.6719 \\ 576.8404 & 584.7931 & 591.7557 \\ 594.7726 & 598.7675 & 609.7118 \\ 629.8092 & 630.7388 & 641.8199 \\ 648.3971 & 658.8952 & 665.6062 \\ 672.2364 & 676.3110 & 679.2894 \\ 684.4763 & 687.1394 & 690.4605 \\ 701.9472 & 707.9999 & 708.7813 \\ 713.9555 & 720.5437 & 724.3569 \\ 728.9098 & 735.7393 & 739.9583 \\ 746.8926 & 749.8506 & 751.8794 \\ 759.0488 & 761.5189 & 770.1465 \\ 772.0450 & 777.9309 & 781.3433 \\ 792.8279 & 798.0131 & 802.3845 \\ 813.1654 & 827.7209 & 833.8570 \\ 853.4097 & 861.4500 & 862.9442 \\ 870.8681 & 889.5063 & 910.0642 \\ 916.2125 & 919.5240 & 923.8621 \\ 934.5115 & 942.7035 & 946.6814 \\ 961.6587 & 968.9178 & 1026.8091 \\ 1032.2337 & 1058.7378 & 1070.9655 \\ 1162.9195 & 1264.8202 & 1273.5781 \\ 1292.9971 & 1347.6915 & 1623.7112 \\ 1631.9877 & 1654.9237 & 1655.5733 \\ 1656.4716 & 1664.4784 & 1666.6966 \\ 1667.3909 & 1668.4592 & 1669.5511 \\ 1671.9283 & 1676.5360 & 1677.1698 \\ 1679.1068 & 1684.7500 & 1688.8092 \\ 1691.5836 & 1692.8087 & 1696.3109 \\ 1697.7049 & 1703.1004 & 1703.7142 \\ 1708.9110 & 1716.4304 & 1720.9780 \\ 1724.9415 & 1725.8466 & 1734.4967 \\ 1735.7149 & 1738.6206 & 2731.2403 \\ 3066.2058 & 3074.8041 & 3100.3870 \\ 3199.0756 & 3215.3283 & 3249.9864 \\ 3301.5269 & 3318.1188 & 3329.5014 \\ 3350.0306 & 3377.9426 & 3391.6677 \\ 3436.8101 & 3454.5657 & 3461.0578 \\ 3483.2468 & 3493.2053 & 3516.6983 \\ 3524.6401 & 3525.8697 & 3533.1306 \\ 3559.0824 & 3561.4084 & 3573.0608 \\ 3585.5850 & 3585.8864 & 3587.3006 \\ 3593.4910 & 3601.2218 & 3609.0310\end{array}$




$\begin{array}{lll}3618.0829 & 3624.1734 & 3625.5726 \\ 3630.7299 & 3631.9499 & 3639.1674 \\ 3646.9933 & 3650.3640 & 3653.2041 \\ 3655.8795 & 3668.5300 & 3670.0370 \\ 3683.3477 & 3692.1042 & 3693.8277 \\ 3694.6931 & 3707.5970 & 3708.1947 \\ 3711.3797 & 3741.2914 & 3745.0075 \\ 3773.4150 & 3806.2244 & 3847.1549 \\ 3861.3956 & 3866.8538 & 3871.4351 \\ 3873.8987 & 3874.6260 & 3881.0434 \\ 3882.3324 & 3890.9624 & 3896.6846\end{array}$

\section{Calcite-Super-Molecule}

3.1. Calcite-Super-Molecule, Optimized Geometry (HF/3-21G)
C $\quad 0.0239840$
$-0.0040300$
$-0.0029460$
O -0.0248470
$-1.2906920-0.0035470$
O 1.1506510
0.6172630
$-0.0479880$
O -1.0825580
0.6700020
0.0345690
Ca -1.3311480
2.4749540
$-1.3933670$
Ca 2.9310750
$-0.1844980 \quad-1.5050480$
Ca 1.5327760
2.4766970
1.4785120
Ca -1.5657850
$-2.4071340$
$-1.4575210$
Ca $\quad-2.8626270$
0.0518850
1.4429340
Ca 1.3565060
$-2.5183300$
1.4685980
$\begin{array}{lll}\text { O } & -0.4582430 & -3.1458930\end{array}$
2.8871250
O 3.8217330
$-5.8371680$
2.8973710
O $\quad 6.9069400$
$-0.9507460$
$-2.8665030$
O $\quad-4.3551710$
$-5.5032730$
$-2.9388640$
O $\quad-2.6794680$
6.3935740
$-2.7262000$
C $\quad-0.1892950$
$-5.0396830$
0.0003550
O 0.2212940
$-3.1816370$
$-2.8929640$
O $\quad-2.0322030$
$-5.6225170$
$-2.6372350$
O $\quad-0.2381390$
$-6.3334450$
0.0026130
O 1.5752710
$-5.7490540$
2.8943680
$\begin{array}{lll}\text { C } & -4.4636720 & -2.3519290\end{array}$
$-0.0098780$
O $\quad-4.0530820$
$-0.4938830$
$-2.9031970$
O $\quad-3.0498720$
$-3.6411350$
$-2.8962820$
C $\quad-3.0987150$
$-4.9348970$
$-2.8940240$
O -1.2853050
$-4.3505050$
$-0.0022690$
O $\quad-4.5125150$
$-3.6456910$
$-0.0076190$
O $\quad-2.6991060$
$-3.0613000$
2.8841360
O $\quad-5.5596820$
$-1.6627510$
$-0.0125020$
O -6.9734820
$-0.3735450$
2.8739030
C $\quad 4.2755660$
$-2.6818390$
0.0017800
O 4.6861560
$-0.8237930$
$-2.8915390$
O 2.4621570
$-3.2662310$
$-2.8899750$
O 4.2267230
$-3.9756010$
0.0040380
O 0.4117800
1.8639610
$-2.9017720$
O $\quad-1.8122190$
$-0.5784760$
$-2.9002080$
O 1.4149900
$-1.2832910$
$-2.8948570$
C 1.3661470
$-2.5770530$
$-2.8925990$
O 0.9555580
$-4.4350990$
0.0007200 


\begin{tabular}{llll}
\hline & & & \\
$\mathrm{O}$ & 3.1795570 & -1.9926620 & -0.0008440 \\
$\mathrm{O}$ & 1.7657560 & -0.7034560 & 2.8855610 \\
$\mathrm{O}$ & 2.7689670 & -3.8507080 & 2.8924750 \\
$\mathrm{C}$ & 2.7201240 & -5.1444700 & 2.8947340 \\
$\mathrm{C}$ & -4.2731860 & 2.6936690 & -0.0186850 \\
$\mathrm{O}$ & -3.8625970 & 4.5517150 & -2.9120040 \\
$\mathrm{O}$ & -2.8593860 & 1.4044630 & -2.9050900 \\
$\mathrm{C}$ & -2.9082290 & 0.1107010 & -2.9028320 \\
$\mathrm{O}$ & -3.3188190 & -1.7473450 & -0.0095120 \\
$\mathrm{O}$ & -4.3220290 & 1.3999070 & -0.0164270 \\
$\mathrm{O}$ & -2.5086200 & 1.9842980 & 2.8753280 \\
$\mathrm{O}$ & -4.7326190 & -0.4581390 & 2.8768920 \\
$\mathrm{O}$ & -1.5054090 & -1.1629530 & 2.8822430 \\
$\mathrm{C}$ & -1.5542520 & -2.4567150 & 2.8845010 \\
$\mathrm{O}$ & -5.3691960 & 3.3828470 & -0.0213090 \\
$\mathrm{O}$ & -5.7797860 & 1.5248010 & 2.8720100 \\
$\mathrm{C}$ & -5.8286290 & 0.2310390 & 2.8742690 \\
$\mathrm{C}$ & 4.4660520 & 2.3637590 & -0.0070270 \\
$\mathrm{O}$ & 2.6526430 & 1.7793670 & -2.8987820 \\
$\mathrm{O}$ & 5.8798520 & 1.0745530 & -2.8934320 \\
$\mathrm{C}$ & 5.8310090 & -0.2192090 & -2.8911740 \\
$\mathrm{O}$ & 5.4204200 & -2.0772550 & 0.0021460 \\
$\mathrm{O}$ & 4.4172090 & 1.0699970 & -0.0047690 \\
$\mathrm{O}$ & 4.0066190 & -0.7880490 & 2.8885500 \\
$\mathrm{C}$ & 0.1916760 & 5.0515130 & -0.0172600 \\
$\mathrm{O}$ & -1.6217340 & 4.4671210 & -2.9090150 \\
$\mathrm{O}$ & 1.6054760 & 3.7623070 & -2.9036650 \\
$\mathrm{C}$ & 1.5566330 & 2.4685450 & -2.9014060 \\
$\mathrm{O}$ & 3.3700420 & 3.0529360 & -0.0096510 \\
$\mathrm{O}$ & 0.1428330 & 3.7577510 & -0.0150020 \\
$\mathrm{O}$ & 1.9562420 & 4.3421420 & 2.8767540 \\
$\mathrm{O}$ & -0.2677570 & 1.8997050 & 2.8783180 \\
$\mathrm{O}$ & 2.9594530 & 1.1948900 & 2.8836680 \\
$\mathrm{C}$ & 2.9106090 & -0.0988720 & 2.8859270 \\
$\mathrm{C}$ & -2.7177440 & 5.1562990 & -2.9116390 \\
$\mathrm{O}$ & -3.1283330 & 3.2982530 & -0.0183200 \\
$\mathrm{O}$ & -0.9043340 & 5.7406910 & -0.0198840 \\
$\mathrm{O}$ & -1.3149240 & 3.8826440 & 2.8734360 \\
$\mathrm{C}$ & -1.3637670 & 2.5888830 & 2.8756940 \\
$\mathrm{O}$ & 5.6109050 & 2.9683430 & -0.0066620 \\
$\mathrm{O}$ & 4.1971050 & 4.2575480 & 2.8797430 \\
$\mathrm{O}$ & 1.3365290 & 5.6560970 & -0.0168940 \\
$\mathrm{O}$ & 3.1499380 & 6.2404880 & 2.8748610 \\
$\mathrm{C}$ & 3.1010950 & 4.9467260 & 2.8771190 \\
$\mathrm{H}$ & 0.1307550 & 0.5696050 & -3.9024060 \\
$\mathrm{H}$ & -0.5504140 & -0.1784670 & -3.9019270 \\
$\mathrm{H}$ & 0.4380190 & -0.3943390 & -3.9002880 \\
$\mathrm{H}$ & 4.4051310 & -2.1181490 & -3.8921730 \\
$\mathrm{H}$ & 3.7239620 & -2.8662210 & -3.8916940 \\
$\mathrm{H}$ & -0.0597310 & -4.4759930 & -3.8935990 \\
$\mathrm{H}$ & -0.6020950 & -5.1854550 & -3.3579080 \\
$\mathrm{H}$ & -4.3341070 & -1.7882380 & -3.9038310 \\
$\mathrm{H}$ & -4.0268430 & -2.7521820 & -3.9017130
\end{tabular}




$\begin{array}{llll} & & & \\ \mathrm{H} & -4.1436220 & 3.2573600 & -3.9126380 \\ \mathrm{H} & -3.8363570 & 2.2934160 & -3.9105210 \\ \mathrm{H} & -3.9270270 & -2.5638020 & 3.8842880 \\ \mathrm{H} & -4.5498540 & -1.7665030 & 3.8820700 \\ \mathrm{H} & -3.7365410 & 2.4817960 & 3.8754810 \\ \mathrm{H} & -4.7384380 & 2.3410610 & 3.8744650 \\ \mathrm{H} & 0.7283200 & 4.8396400 & 3.8769060 \\ \mathrm{H} & -0.2735760 & 4.6989040 & 3.8758900 \\ \mathrm{H} & 0.5378350 & -0.2059580 & 3.8857130 \\ \mathrm{H} & -0.4640620 & -0.3466940 & 3.8846970 \\ \mathrm{H} & -0.0849920 & 0.5913410 & 3.8834950 \\ \mathrm{H} & 0.3473490 & -5.2515560 & 3.8945210 \\ \mathrm{H} & -0.2754780 & -4.4542570 & 3.8923020 \\ \mathrm{H} & 4.0008000 & 2.0111500 & 3.8861220 \\ \mathrm{H} & 4.3798700 & 2.9491850 & 3.8849200 \\ \mathrm{H} & 3.8103150 & -3.0344480 & 3.8949300 \\ \mathrm{H} & 4.1893840 & -2.0964130 & 3.8937280 \\ \mathrm{H} & -2.0826890 & -6.9220290 & -1.6055760 \\ \mathrm{H} & -1.2794860 & -7.1497050 & -0.9998410 \\ \mathrm{H} & -5.5538630 & -4.4619510 & -1.0100740 \\ \mathrm{H} & -5.7424470 & -0.3543870 & -1.0176790 \\ \mathrm{H} & -5.3633770 & 0.5836470 & -1.0188810 \\ \mathrm{H} & -5.2810040 & 0.0036150 & -1.9030450 \\ \mathrm{H} & 3.1872770 & 4.3613000 & -1.0148280 \\ \mathrm{H} & 2.5644500 & 5.1585990 & -1.0170470 \\ \mathrm{H} & 2.6468240 & 4.5785670 & -1.9012100 \\ \mathrm{H} & -1.0870990 & 7.0490540 & -1.0250610 \\ \mathrm{H} & -5.5519610 & 4.6912110 & -1.0264860 \\ \mathrm{H} & -5.0905180 & 5.0492130 & -1.9118520 \\ \mathrm{H} & 6.6483410 & -2.5747530 & -0.9980070 \\ \mathrm{H} & 6.8388270 & 2.4708450 & -1.0068140 \\ \mathrm{H} & -2.1661390 & 5.3406810 & 0.9818350 \\ \mathrm{H} & 6.9212000 & 1.8908130 & -1.8909780 \\ \mathrm{H} & 5.8919300 & 4.2626980 & 0.9939720 \\ \mathrm{H} & 5.4589100 & 4.6575580 & 1.8780240 \\ \mathrm{H} & 1.6175540 & 6.9504520 & 0.9837400 \\ \mathrm{H} & 2.1729670 & 7.1294400 & 1.8694300 \\ \mathrm{H} & 5.7014450 & -0.7829000 & 1.0027800 \\ \mathrm{H} & 5.3941800 & 0.1810440 & 1.0006620 \\ \mathrm{H} & 5.2684240 & -0.3880400 & 1.8868310 \\ \mathrm{H} & 5.2036940 & -4.8645540 & 1.0094690 \\ \mathrm{H} & 0.7388330 & -7.2223970 & 1.0080440 \\ \mathrm{H} & 1.2942460 & -7.0434090 & 1.8937340 \\ \mathrm{H} & -2.5471100 & -4.7505150 & 0.9994500 \\ \mathrm{H} & -3.5355440 & -4.5346430 & 0.9978110 \\ \mathrm{H} & -2.9801310 & -4.3556550 & 1.8835020 \\ \mathrm{H} & -6.8214870 & -2.0627600 & 0.9892180 \\ \mathrm{H} & -6.6310010 & -1.6679010 & 1.8732690 \\ & -2.9828380 & 0.9804100 \\ & -4.791870 & 1.8680050 \\ \mathrm{H} & & \\ \mathrm{H} & & \\ \mathrm{H} & & \end{array}$




$\begin{array}{llll}\mathrm{H} & 2.1834790 & -4.9325970 & -0.9994320 \\ \mathrm{H} & 2.6449220 & -4.5745940 & -1.8847980 \\ \mathrm{H} & -3.5598700 & 7.4207260 & -3.6882040 \\ \mathrm{H} & -2.0666420 & 7.0252400 & -1.3187040 \\ \mathrm{H} & -0.7380330 & 4.5140220 & -4.3207500 \\ \mathrm{H} & 0.7988680 & 4.8466880 & -3.8675640 \\ \mathrm{H} & 3.8820690 & 2.0193680 & -3.9880530 \\ \mathrm{H} & 4.6796960 & 1.8724560 & -3.7171890 \\ \mathrm{H} & -8.2224940 & 0.1725250 & 3.8212030 \\ \mathrm{H} & -4.4260650 & -6.8549250 & -3.8844530 \\ \mathrm{H} & -5.5666300 & -4.9485040 & -1.8635230 \\ \mathrm{H} & 7.8699580 & -0.5637470 & -4.1620440 \\ \mathrm{H} & 7.3005350 & -2.2340590 & -1.8898810 \\ \mathrm{H} & 3.8656570 & -6.8031440 & 4.2466530 \\ \mathrm{H} & 4.2902070 & 6.9574440 & 3.8450990 \\ \mathrm{H} & 5.0862830 & -6.1608230 & 1.8475000\end{array}$

Frequencies (not scaled) C-12( $1^{\text {st }}$ atom $) \mathrm{O} 16\left(2^{\text {nd }}\right.$ atom):

$\begin{array}{lll}-7119.4749 & -6335.0897 & -4612.2910 \\ -4099.4411 & -3290.1844 & -3113.3856 \\ -2910.7267 & -2797.2946 & -2584.6316 \\ -2484.6773 & -2274.1030 & -2195.4768 \\ -1875.5031 & -1802.0149 & -1706.2285 \\ -1616.6087 & -1609.3139 & -1579.0980 \\ -1564.7671 & -1462.3237 & -1436.6981 \\ -1405.6596 & -1389.4577 & -1374.7159 \\ -1356.0239 & -1318.7415 & -1282.2220 \\ -1271.2282 & -1250.8088 & -1239.5103 \\ -1212.0734 & -1207.5609 & -1153.3111 \\ -1133.4841 & -1104.2644 & -1096.1428 \\ -1083.8243 & -1001.2616 & -932.2509 \\ -917.0034 & -883.2829 & -705.0557 \\ -693.0334 & -629.6991 & -540.6487 \\ -481.7557 & -420.8576 & -409.8951 \\ -367.1602 & -359.8938 & -354.5024 \\ -343.9913 & -333.1626 & -321.0211 \\ -305.7551 & -272.5993 & -266.8181 \\ -239.9516 & -168.5247 & -154.4909 \\ -147.5029 & -135.1016 & -118.5407 \\ -114.3244 & -103.5198 & -98.6072 \\ -95.8262 & -84.8528 & -76.3946 \\ -74.2211 & -62.3723 & -58.4392 \\ -50.8934 & -47.9879 & -39.9054 \\ -11.9347 & 11.1668 & 33.4928 \\ 41.8830 & 50.9704 & 55.9996 \\ 57.6356 & 64.4449 & 67.2111 \\ 69.2846 & 69.7560 & 72.6742 \\ 76.5225 & 82.8870 & 87.2375 \\ 88.9387 & 91.9419 & 94.3886 \\ 94.7105 & 99.7505 & 103.9816 \\ 105.5742 & 108.6130 & 109.9995 \\ 111.4996 & 113.5123 & 115.1019 \\ 116.1726 & 119.6297 & 121.0665 \\ 123.3851 & 126.3002 & 126.8384\end{array}$




$\begin{array}{lll}128.0487 & 130.7127 & 132.5017 \\ 133.9287 & 136.4166 & 138.5402 \\ 142.0015 & 142.6964 & 145.8531 \\ 146.7540 & 150.0127 & 151.5218 \\ 153.8898 & 155.6530 & 158.9060 \\ 160.9770 & 162.1721 & 162.3163 \\ 163.6868 & 167.1066 & 168.7441 \\ 172.1182 & 174.0056 & 175.3668 \\ 176.8814 & 178.0641 & 180.8901 \\ 184.2982 & 185.1016 & 185.8089 \\ 190.6645 & 191.0948 & 193.2214 \\ 195.8195 & 197.9006 & 201.8410 \\ 204.0788 & 204.1655 & 211.3876 \\ 212.8605 & 214.8272 & 217.0039 \\ 222.4640 & 224.7333 & 228.1320 \\ 232.6073 & 238.3250 & 242.3994 \\ 242.8380 & 246.6923 & 252.1570 \\ 253.7437 & 258.9818 & 262.0937 \\ 265.7659 & 268.8332 & 272.6238 \\ 273.4682 & 275.1360 & 278.3844 \\ 280.4970 & 283.5381 & 287.0417 \\ 288.0843 & 289.7196 & 296.5390 \\ 300.0978 & 303.9137 & 305.9763 \\ 313.3582 & 315.8797 & 321.9167 \\ 323.3901 & 328.0783 & 329.3704 \\ 331.6238 & 334.1869 & 340.3055 \\ 346.7872 & 355.3688 & 359.8604 \\ 361.4690 & 371.6188 & 386.3572 \\ 388.6562 & 398.0802 & 411.4954 \\ 442.4663 & 443.8085 & 465.8282 \\ 476.9125 & 485.8491 & 493.8297 \\ 499.2372 & 511.9038 & 520.3760 \\ 548.2306 & 553.0542 & 554.6815 \\ 557.7454 & 566.0371 & 571.6724 \\ 584.8839 & 591.0712 & 594.7122 \\ 598.5219 & 601.2440 & 604.0671 \\ 605.2453 & 612.8377 & 623.5147 \\ 629.3572 & 638.1712 & 638.9903 \\ 641.0493 & 653.0980 & 656.8783 \\ 659.6635 & 672.6584 & 676.5017 \\ 680.2590 & 682.7856 & 686.3596 \\ 689.9051 & 691.7900 & 693.8527 \\ 700.5945 & 703.4392 & 704.6559 \\ 708.7670 & 710.3649 & 711.9666 \\ 712.6669 & 714.3897 & 715.7365 \\ 716.9028 & 717.2845 & 721.0527 \\ 726.1001 & 726.5409 & 732.1504 \\ 732.5116 & 737.9164 & 739.5158 \\ 744.4629 & 749.1055 & 752.1170 \\ 754.9941 & 758.7997 & 761.6198 \\ 769.2202 & 771.5518 & 783.6097 \\ 787.4632 & 799.6283 & 804.7830 \\ 813.2503 & 828.0115 & 856.6427\end{array}$




$\begin{array}{lll}861.5665 & 861.8345 & 866.6248 \\ 868.0142 & 873.0571 & 876.8846 \\ 878.6489 & 886.9339 & 891.1910 \\ 897.9691 & 899.0272 & 908.0479 \\ 911.0700 & 911.9038 & 916.0802 \\ 916.9843 & 921.9695 & 924.3112 \\ 925.6484 & 926.1968 & 930.2577 \\ 933.0948 & 937.1478 & 940.2654 \\ 950.3734 & 957.6485 & 963.9143 \\ 968.2567 & 974.3212 & 975.6495 \\ 980.6748 & 983.5239 & 989.4668 \\ 991.5716 & 992.3817 & 994.2718 \\ 998.2363 & 1000.0858 & 1002.7877 \\ 1007.4140 & 1008.7540 & 1010.8813 \\ 1013.8884 & 1016.4884 & 1020.4653 \\ 1023.0620 & 1024.4193 & 1026.1171 \\ 1028.3381 & 1030.0443 & 1030.4639 \\ 1034.7184 & 1040.5377 & 1042.7378 \\ 1044.9224 & 1046.8032 & 1052.7064 \\ 1055.5189 & 1058.5221 & 1065.5885 \\ 1073.7381 & 1076.4502 & 1078.5662 \\ 1087.3704 & 1087.7600 & 1088.5296 \\ 1094.6638 & 1096.7953 & 1101.2188 \\ 1107.5242 & 1115.2578 & 1117.6842 \\ 1120.9879 & 1122.4232 & 1125.1344 \\ 1134.2680 & 1135.8969 & 1138.3060 \\ 1141.3521 & 1147.9411 & 1151.5340 \\ 1156.8247 & 1167.1337 & 1167.9730 \\ 1173.9209 & 1174.3200 & 1177.8211 \\ 1178.5978 & 1179.1401 & 1183.0835 \\ 1188.5607 & 1193.9000 & 1199.6728 \\ 1207.5973 & 1217.6884 & 1222.7309 \\ 1241.7807 & 1244.2048 & 1251.4307 \\ 1267.4445 & 1270.8208 & 1277.3226 \\ 1284.6786 & 1289.2501 & 1318.6103 \\ 1322.4921 & 1330.1683 & 1330.9363 \\ 1334.3701 & 1347.2255 & 1349.8706 \\ 1361.5645 & 1374.5999 & 1384.6346 \\ 1389.0825 & 1394.4690 & 1396.5802 \\ 1401.5948 & 1402.7298 & 1405.9033 \\ 1414.5654 & 1420.5968 & 1420.8648 \\ 1428.0645 & 1429.1456 & 1439.6258 \\ 1444.1272 & 1446.7907 & 1451.3411 \\ 1459.3400 & 1463.9360 & 1468.1330 \\ 1474.8899 & 1478.8954 & 1482.5172 \\ 1482.8801 & 1484.2882 & 1488.9261 \\ 1492.6853 & 1497.0152 & 1499.5205 \\ 1504.0920 & 1508.2490 & 1512.0709 \\ 1513.5367 & 1516.1709 & 1522.1745 \\ 1523.5889 & 1524.1515 & 1525.1282 \\ 1528.3091 & 1528.5434 & 1534.7197 \\ 1536.7372 & 1542.6257 & 1546.7982 \\ 1549.2404 & 1551.4928 & 1554.6807\end{array}$




\begin{tabular}{|c|c|c|}
\hline 1557.0856 & 1561.7647 & 1564.2617 \\
\hline 1567.2605 & 1568.5578 & 1571.6594 \\
\hline 1579.6686 & 1580.1270 & 1582.2919 \\
\hline 1585.2333 & 1586.2137 & 1592.0404 \\
\hline 1600.4244 & 1603.1718 & 1604.8824 \\
\hline 1615.5937 & 1617.8312 & 1628.1017 \\
\hline 1643.7996 & 1649.8148 & 1651.1021 \\
\hline 1655.7770 & 1659.6966 & 1661.6017 \\
\hline 1672.2366 & 1673.7823 & 1678.9429 \\
\hline 1690.7486 & 1695.1116 & 1702.7114 \\
\hline 1707.2381 & 1717.6136 & 1721.6222 \\
\hline 1724.5166 & 1726.1274 & 1728.2765 \\
\hline 1752.3313 & 1778.0554 & 1802.7602 \\
\hline 1809.1477 & 1811.5290 & 1831.2270 \\
\hline 1834.3375 & 1873.7979 & 1894.9971 \\
\hline 1900.4094 & 2856.4555 & 9814.0644 \\
\hline \multicolumn{3}{|c|}{ Frequencies (not scaled) $\mathrm{C}-12\left(1^{\text {st }}\right.$ atom $) \mathrm{O} 18\left(2^{\text {nd }}\right.$ atom): } \\
\hline-7119.4675 & -6335.0834 & -4612.2766 \\
\hline-4099.4395 & -3290.1774 & -3113.3827 \\
\hline-2910.7144 & -2797.2937 & -2584.6287 \\
\hline-2484.6708 & -2274.1009 & -2195.4739 \\
\hline-1875.4973 & -1802.0112 & -1706.2283 \\
\hline-1616.6087 & -1609.3134 & -1579.0975 \\
\hline-1564.7669 & -1462.3236 & -1436.6975 \\
\hline-1405.6559 & -1389.4543 & -1374.7112 \\
\hline-1356.0233 & -1318.7400 & -1282.2202 \\
\hline-1271.2282 & -1250.8072 & -1239.5079 \\
\hline-1212.0706 & -1207.5587 & -1153.3084 \\
\hline-1133.4834 & -1104.2521 & -1096.1420 \\
\hline-1083.8150 & -1001.2536 & -932.2492 \\
\hline-916.9973 & -883.2709 & -705.0464 \\
\hline-693.0287 & -629.6941 & -540.6485 \\
\hline-481.7555 & -420.8570 & -409.8927 \\
\hline-367.1548 & -359.8937 & -354.5023 \\
\hline-343.9905 & -333.1622 & -321.0190 \\
\hline-305.7536 & -272.5975 & -266.8177 \\
\hline-239.9504 & -168.5222 & -154.4908 \\
\hline-147.5006 & -135.1013 & -118.5407 \\
\hline-114.3220 & -103.5188 & -98.6028 \\
\hline-95.8239 & -84.8506 & -76.3895 \\
\hline-74.2201 & -62.3594 & -58.4319 \\
\hline-50.8912 & -47.9814 & -39.9041 \\
\hline-11.9367 & 11.1706 & 33.4903 \\
\hline 41.8760 & 50.9606 & 55.9893 \\
\hline 57.6044 & 64.4431 & 67.1915 \\
\hline 69.1739 & 69.7433 & 72.6700 \\
\hline 76.4957 & 82.8712 & 87.2238 \\
\hline 88.9199 & 91.8774 & 94.3467 \\
\hline 94.7004 & 99.7346 & 103.8974 \\
\hline 105.5537 & 108.5944 & 109.8949 \\
\hline 111.4339 & 113.4856 & 115.0420 \\
\hline 115.9141 & 119.5844 & 121.0510 \\
\hline 123.3743 & 126.0615 & 126.8009 \\
\hline
\end{tabular}




$\begin{array}{lll} & & \\ 127.9900 & 130.6174 & 132.3509 \\ 133.9064 & 136.3686 & 138.5015 \\ 141.9952 & 142.6625 & 145.7082 \\ 146.5172 & 149.9133 & 151.5013 \\ 153.7571 & 155.5805 & 158.8475 \\ 160.7318 & 162.0555 & 162.2700 \\ 163.6742 & 166.9213 & 168.6382 \\ 172.0868 & 173.8865 & 175.3203 \\ 176.8591 & 178.0276 & 180.8683 \\ 184.1107 & 185.0505 & 185.7222 \\ 190.1964 & 190.9692 & 193.1843 \\ 195.2653 & 197.7547 & 201.7520 \\ 203.2376 & 204.0839 & 209.9418 \\ 212.6798 & 214.3797 & 216.9661 \\ 222.3600 & 224.6632 & 227.9746 \\ 232.3882 & 237.6534 & 242.1943 \\ 242.3971 & 246.2716 & 251.9593 \\ 253.7302 & 258.9185 & 262.0834 \\ 265.6888 & 268.6562 & 272.5822 \\ 273.1908 & 275.0953 & 278.3099 \\ 280.4589 & 283.0670 & 286.8094 \\ 287.9538 & 289.6662 & 296.5255 \\ 300.0285 & 303.8226 & 305.9629 \\ 313.2741 & 315.8073 & 321.8848 \\ 323.3075 & 327.9395 & 329.0168 \\ 331.3637 & 333.9400 & 340.0470 \\ 344.8204 & 351.2311 & 357.8586 \\ 360.8405 & 371.4742 & 386.1873 \\ 386.9928 & 398.0743 & 411.1600 \\ 442.4611 & 443.7893 & 465.8202 \\ 476.8992 & 485.8389 & 493.8115 \\ 499.1891 & 511.9024 & 520.3721 \\ 548.2299 & 553.0511 & 554.6773 \\ 557.7442 & 566.0370 & 571.6720 \\ 584.8788 & 591.0666 & 594.7117 \\ 598.5218 & 601.2389 & 604.0655 \\ 605.2450 & 612.8374 & 623.5134 \\ 629.3565 & 638.1681 & 638.9887 \\ 641.0467 & 653.0898 & 656.8780 \\ 659.6621 & 672.6556 & 676.5017 \\ 680.2580 & 682.7834 & 686.3588 \\ 689.8786 & 691.7871 & 693.8510 \\ 700.5652 & 703.4192 & 704.5951 \\ 708.7627 & 710.3550 & 711.9068 \\ 712.6604 & 714.3749 & 715.7303 \\ 716.8987 & 717.2716 & 720.9279 \\ 726.0578 & 726.5265 & 731.9791 \\ 732.4748 & 737.7241 & 739.5119 \\ 744.3129 & 746.5112 & 749.1401 \\ 752.2938 & 755.3755 & 756.2884 \\ 760.6260 & 771.3569 & 783.5829 \\ 787.4104 & 799.6245 & 804.7807 \\ 813.2366 & 828.0112 & 856.6410\end{array}$




$\begin{array}{lll}861.5652 & 861.8327 & 866.6171 \\ 868.0115 & 873.0558 & 876.8712 \\ 878.6484 & 886.9335 & 891.1887 \\ 897.9653 & 899.0215 & 908.0415 \\ 911.0203 & 911.8761 & 916.0710 \\ 916.9714 & 921.9381 & 924.3092 \\ 925.5603 & 926.1826 & 930.1696 \\ 933.0587 & 937.1434 & 940.2649 \\ 950.3727 & 954.0178 & 963.9115 \\ 968.2231 & 974.3208 & 975.6488 \\ 980.6732 & 983.4225 & 989.4443 \\ 991.5665 & 992.3644 & 994.2702 \\ 998.2337 & 1000.0838 & 1002.7784 \\ 1007.4127 & 1008.7536 & 1010.8807 \\ 1013.8832 & 1016.4879 & 1020.4626 \\ 1023.0563 & 1024.4183 & 1026.1152 \\ 1028.3310 & 1030.0407 & 1030.4623 \\ 1034.7159 & 1040.5346 & 1042.7373 \\ 1044.9223 & 1046.8027 & 1052.7026 \\ 1055.5180 & 1058.5178 & 1065.5871 \\ 1073.7357 & 1076.4480 & 1078.5544 \\ 1087.3547 & 1087.7569 & 1088.5285 \\ 1094.6593 & 1096.7869 & 1101.2186 \\ 1107.4929 & 1112.8235 & 1115.2712 \\ 1117.7016 & 1121.0661 & 1122.5695 \\ 1125.1465 & 1135.8843 & 1138.2869 \\ 1141.3427 & 1147.9410 & 1151.5181 \\ 1156.8165 & 1167.1293 & 1167.9698 \\ 1173.9175 & 1174.3195 & 1177.8202 \\ 1178.5960 & 1179.1390 & 1183.0827 \\ 1188.5603 & 1193.8998 & 1199.6708 \\ 1207.5934 & 1217.6869 & 1222.7304 \\ 1241.7803 & 1244.1943 & 1251.4281 \\ 1267.4352 & 1270.8189 & 1277.3221 \\ 1284.6779 & 1289.2396 & 1318.6060 \\ 1322.4892 & 1330.1662 & 1330.9354 \\ 1334.3693 & 1347.2252 & 1349.8705 \\ 1361.5478 & 1374.5985 & 1384.6300 \\ 1389.0806 & 1394.4504 & 1396.5720 \\ 1401.5923 & 1402.7271 & 1405.9028 \\ 1414.5568 & 1420.5723 & 1420.8639 \\ 1428.0591 & 1429.1188 & 1439.6194 \\ 1444.0904 & 1446.7613 & 1451.3411 \\ 1459.3385 & 1463.9320 & 1468.1021 \\ 1474.8595 & 1478.8931 & 1482.5152 \\ 1482.8738 & 1484.2757 & 1488.9218 \\ 1492.6050 & 1496.9771 & 1499.5194 \\ 1504.0867 & 1508.1791 & 1512.0707 \\ 1513.5310 & 1515.9426 & 1522.1673 \\ 1523.5817 & 1524.1020 & 1525.1272 \\ 1528.2746 & 1528.4827 & 1534.7137 \\ 1536.7259 & 1542.5853 & 1546.7870 \\ 1549.2382 & 1551.1014 & 1554.4409\end{array}$




\begin{tabular}{lll}
1556.9701 & 1561.3978 & 1563.7261 \\
1567.0709 & 1568.3140 & 1571.1583 \\
1579.6360 & 1580.0139 & 1581.7254 \\
1585.1009 & 1586.1972 & 1591.9971 \\
1598.7239 & 1600.8220 & 1604.3641 \\
1615.4694 & 1617.5474 & 1622.7576 \\
1643.5101 & 1649.8140 & 1651.1011 \\
1655.7739 & 1659.6885 & 1661.2582 \\
1672.2338 & 1673.7792 & 1678.9350 \\
1690.7191 & 1695.1106 & 1702.7111 \\
1707.2236 & 1717.6134 & 1721.6171 \\
1724.5154 & 1726.1274 & 1728.2765 \\
1752.3254 & 1778.0523 & 1802.7579 \\
1809.1453 & 1811.5094 & 1831.2256 \\
1834.3337 & 1873.7938 & 1894.9964 \\
1900.4051 & 2856.4555 & 9814.0477 \\
Frequencies (not scaled) C-13(1 \\
-7119.4628 & -6335.0656 & -4612.2624 \\
-4099.4388 & -3290.1757 & -3113.3853 \\
-2910.7102 & -2797.2936 & -2584.6264 \\
-2484.6670 & -2274.0967 & -2195.4712 \\
-1875.5012 & -1802.0113 & -1706.2278 \\
-1616.6085 & -1609.3136 & -1579.0978 \\
-1564.7669 & -1462.3236 & -1436.6973 \\
-1405.6578 & -1389.4560 & -1374.7132 \\
-1356.0237 & -1318.7398 & -1282.2212 \\
-1271.2281 & -1250.8081 & -1239.5097 \\
-1212.0729 & -1207.5594 & -1153.3103 \\
-1133.4834 & -1104.2619 & -1096.1386 \\
-1083.8215 & -1001.2507 & -932.2504 \\
-917.0016 & -883.2812 & -705.0552 \\
-693.0329 & -629.6988 & -540.6487 \\
-481.7557 & -420.8576 & -409.8951 \\
-367.1601 & -359.8938 & -354.5024 \\
-343.9913 & -333.1625 & -321.0208 \\
-305.7548 & -272.5989 & -266.8180 \\
-239.9511 & -168.5236 & -154.4894 \\
-147.5016 & -135.1015 & -118.5403 \\
-114.3240 & -103.5197 & -98.6069 \\
-95.8236 & -84.8491 & -76.3932 \\
-74.2209 & -62.3709 & -58.4345 \\
-50.8924 & -47.9852 & -39.9035 \\
-11.9346 & 11.1666 & 33.4898 \\
41.8798 & 50.9614 & 55.9969 \\
57.6324 & 64.4408 & 67.2048 \\
69.2765 & 69.7536 & 72.6717 \\
76.5025 & 82.8778 & 87.2314 \\
88.9359 & 91.9361 & 94.3854 \\
94.7074 & 99.7433 & 103.9623 \\
105.5526 & 108.6048 & 109.9886 \\
111.4886 & 113.5031 & 115.0817 \\
116.1564 & 119.6164 & 121.0634 \\
123.3825 & 126.2723 & 126.8227 \\
& & \\
\hline
\end{tabular}




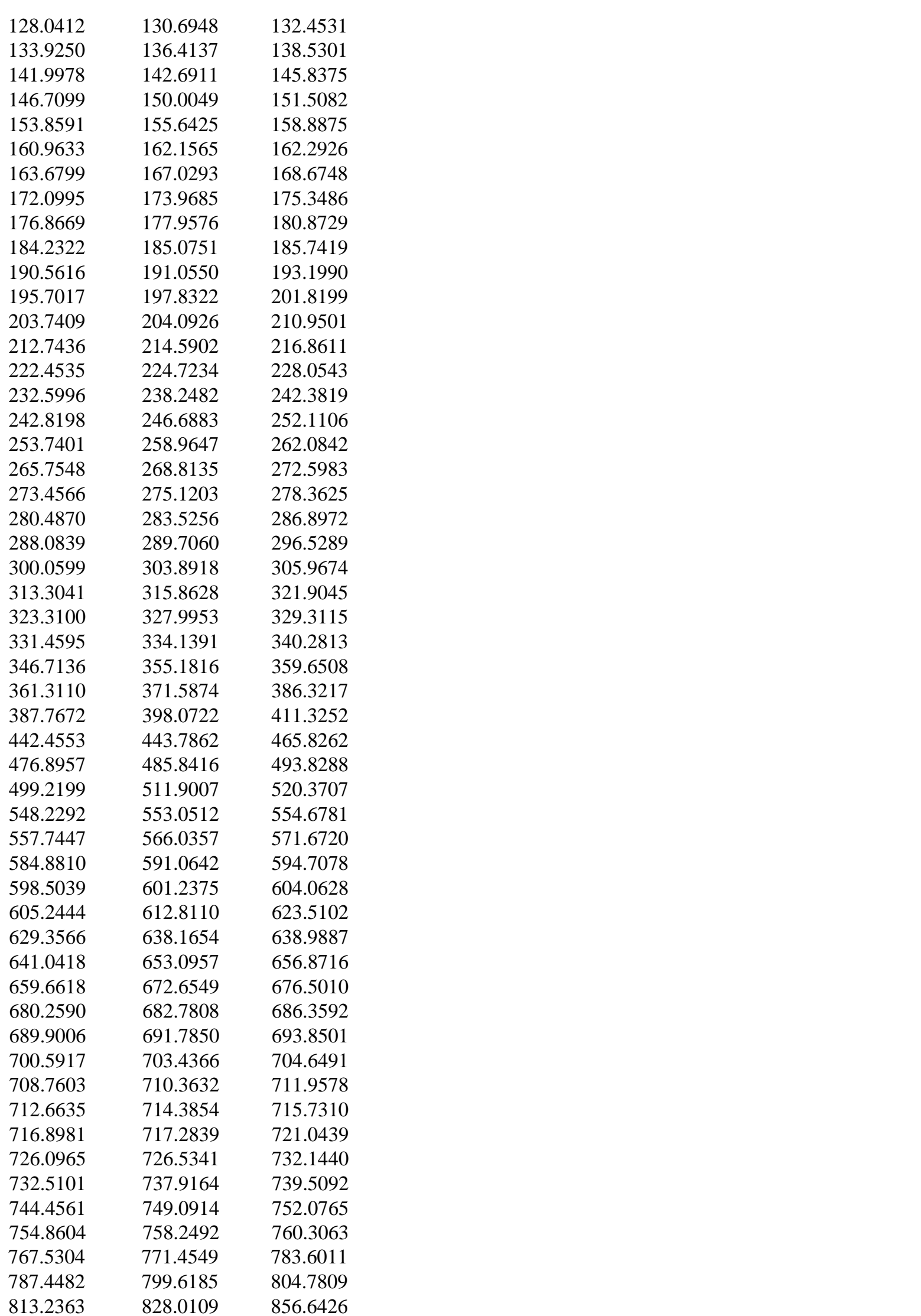




$\begin{array}{lll}861.5656 & 861.8266 & 866.6074 \\ 868.0104 & 873.0401 & 876.8828 \\ 878.6457 & 886.9334 & 891.1678 \\ 897.9593 & 898.9545 & 907.9506 \\ 909.8517 & 911.7339 & 915.8194 \\ 916.7634 & 920.9165 & 923.2725 \\ 924.3456 & 926.1810 & 928.8924 \\ 932.5599 & 937.0136 & 937.9916 \\ 940.2679 & 950.3742 & 963.9094 \\ 968.1686 & 974.3186 & 975.6471 \\ 980.6672 & 983.1538 & 989.3916 \\ 991.5607 & 992.3288 & 994.2684 \\ 998.2226 & 1000.0789 & 1002.7604 \\ 1007.4123 & 1008.7523 & 1010.8804 \\ 1013.8636 & 1016.4879 & 1020.4542 \\ 1023.0386 & 1024.4182 & 1026.1101 \\ 1028.3139 & 1030.0395 & 1030.4594 \\ 1034.7128 & 1040.5290 & 1042.7373 \\ 1044.9207 & 1046.8013 & 1052.6944 \\ 1055.5152 & 1058.5122 & 1065.5807 \\ 1073.7311 & 1076.4402 & 1078.5599 \\ 1087.3687 & 1087.7549 & 1088.5244 \\ 1094.6564 & 1096.7832 & 1101.2138 \\ 1107.5233 & 1115.2562 & 1117.6789 \\ 1120.9741 & 1122.4223 & 1125.1305 \\ 1134.2573 & 1135.8965 & 1138.2965 \\ 1141.3414 & 1147.9401 & 1151.5176 \\ 1156.8208 & 1167.1272 & 1167.9708 \\ 1173.9088 & 1174.3176 & 1177.8181 \\ 1178.5954 & 1179.1397 & 1183.0814 \\ 1188.5582 & 1193.8968 & 1199.6660 \\ 1207.5877 & 1217.6858 & 1222.7286 \\ 1241.7694 & 1244.1924 & 1251.4196 \\ 1267.4264 & 1270.8082 & 1277.3212 \\ 1284.6770 & 1289.2225 & 1318.5955 \\ 1322.4791 & 1330.1616 & 1330.9328 \\ 1334.3432 & 1347.2194 & 1349.8697 \\ 1361.4710 & 1374.5975 & 1384.6220 \\ 1389.0244 & 1394.4090 & 1396.5139 \\ 1401.5418 & 1402.7066 & 1405.8972 \\ 1414.2782 & 1420.4768 & 1420.8497 \\ 1427.9756 & 1429.0141 & 1439.5971 \\ 1443.5894 & 1446.6799 & 1451.3177 \\ 1459.0368 & 1463.7190 & 1467.9827 \\ 1474.6396 & 1478.7697 & 1482.3066 \\ 1482.6715 & 1484.2275 & 1488.8487 \\ 1492.1685 & 1496.8479 & 1499.4883 \\ 1503.3364 & 1507.8473 & 1511.6273 \\ 1513.3841 & 1515.0728 & 1520.1465 \\ 1523.4057 & 1523.8871 & 1525.0903 \\ 1527.4834 & 1528.0867 & 1533.9383 \\ 1535.6261 & 1541.2587 & 1542.1561 \\ 1547.1003 & 1549.2258 & 1551.2700\end{array}$




$\begin{array}{lll}1555.4910 & 1558.2101 & 1562.3738 \\ 1565.8758 & 1567.9335 & 1569.8729 \\ 1575.1994 & 1579.6584 & 1580.2550 \\ 1584.6306 & 1584.9052 & 1586.2304 \\ 1591.6147 & 1592.4390 & 1602.5165 \\ 1610.9930 & 1615.9651 & 1618.6408 \\ 1643.2853 & 1649.8117 & 1651.0875 \\ 1655.7658 & 1659.6764 & 1660.8878 \\ 1672.2295 & 1673.7734 & 1678.9065 \\ 1690.6567 & 1695.0944 & 1702.7064 \\ 1707.2054 & 1717.6130 & 1721.6090 \\ 1724.5153 & 1726.1269 & 1728.2765 \\ 1752.3038 & 1778.0472 & 1802.7473 \\ 1809.1146 & 1811.4886 & 1831.2221 \\ 1834.3072 & 1873.7838 & 1894.9912 \\ 1900.3940 & 2856.4553 & 9814.0150\end{array}$

Frequencies (not scaled) C-13( $\left(1^{\text {st }}\right.$ atom $) \mathrm{O} 18\left(2^{\text {nd }}\right.$ atom $)$ :

$\begin{array}{lll}-7119.4554 & -6335.0592 & -4612.2478\end{array}$

$\begin{array}{lll}-4099.4372 & -3290.1687 & -3113.3824\end{array}$

$\begin{array}{lll}-2910.6977 & -2797.2927 & -2584.6235\end{array}$

$\begin{array}{lll}-2484.6604 & -2274.0945 & -2195.4683\end{array}$

$\begin{array}{lll}-1875.4954 & -1802.0075 & -1706.2275\end{array}$

$-1616.6085 \quad-1609.3130 \quad-1579.0973$

$\begin{array}{lll}-1564.7668 & -1462.3235 & -1436.6967\end{array}$

$\begin{array}{lll}-1405.6540 & -1389.4526 & -1374.7084\end{array}$

$\begin{array}{lll}-1356.0231 & -1318.7383 & -1282.2193\end{array}$

$\begin{array}{lll}-1271.2281 & -1250.8064 & -1239.5072\end{array}$

$\begin{array}{lll}-1212.0700 & -1207.5571 & -1153.3075\end{array}$

$\begin{array}{lll}-1133.4827 & -1104.2496 & -1096.1377\end{array}$

$\begin{array}{lll}-1083.8120 & -1001.2425 & -932.2486\end{array}$

$\begin{array}{lll}-108.9955 & -883.2691 & -705.0459\end{array}$

$\begin{array}{lll}-693.0281 & -629.6938 & -540.6485\end{array}$

$\begin{array}{lll}-481.7555 & -420.8570 & -409.8927\end{array}$

$\begin{array}{lll}-367.1548 & -359.8937 & -354.5023\end{array}$

$\begin{array}{lll}-343.9905 & -333.1621 & -321.0187\end{array}$

$\begin{array}{lll}-305.7533 & -272.5971 & -266.8176\end{array}$

$\begin{array}{lll}-239.9499 & -168.5210 & -154.4892\end{array}$

$\begin{array}{lll}-147.4994 & -135.1012 & -118.5403\end{array}$

$-114.3216 \quad-103.5188 \quad-98.6025$

$\begin{array}{lll}-95.8213 & -84.8469 & -76.3881\end{array}$

$\begin{array}{lll}-74.2199 & -62.3580 & -58.4272\end{array}$

$\begin{array}{lll}-50.8901 & -47.9786 & -39.9022\end{array}$

$\begin{array}{lll}-11.9367 & 11.1704 & 33.4874\end{array}$

$\begin{array}{lll}41.8728 & 50.9514 & 55.9866\end{array}$

$\begin{array}{lll}57.6011 & 64.4390 & 67.1851\end{array}$

$\begin{array}{lll}69.1658 & 69.7407 & 72.6675\end{array}$

$\begin{array}{lll}76.4757 & 82.8618 & 87.2175\end{array}$

$\begin{array}{lll}88.9170 & 91.8715 & 94.3432\end{array}$

$\begin{array}{lll}94.6975 & 99.7272 & 103.8774\end{array}$

$\begin{array}{lll}105.5315 & 108.5856 & 109.8832\end{array}$

$\begin{array}{lll}111.4224 & 113.4762 & 115.0202\end{array}$

$\begin{array}{lll}115.8994 & 119.5711 & 121.0479\end{array}$

$\begin{array}{lll}123.3716 & 126.0236 & 126.7893\end{array}$ 


$\begin{array}{lll}127.9839 & 130.5987 & 132.3067 \\ 133.9036 & 136.3661 & 138.4913 \\ 141.9916 & 142.6569 & 145.6882 \\ 146.4756 & 149.9062 & 151.4886 \\ 153.7238 & 155.5705 & 158.8263 \\ 160.7151 & 162.0379 & 162.2540 \\ 163.6665 & 166.8394 & 168.5717 \\ 172.0664 & 173.8506 & 175.3017 \\ 176.8453 & 177.9176 & 180.8512 \\ 184.0389 & 185.0156 & 185.6553 \\ 190.0841 & 190.9398 & 193.1633 \\ 195.1529 & 197.6853 & 201.7248 \\ 202.9084 & 204.0146 & 209.5604 \\ 212.5861 & 214.1557 & 216.8360 \\ 222.3516 & 224.6545 & 227.9083 \\ 232.3815 & 237.5767 & 242.1871 \\ 242.3799 & 246.2619 & 251.9035 \\ 253.7269 & 258.9026 & 262.0744 \\ 265.6786 & 268.6359 & 272.5556 \\ 273.1776 & 275.0809 & 278.2885 \\ 280.4501 & 283.0607 & 286.6671 \\ 287.9525 & 289.6535 & 296.5162 \\ 299.9887 & 303.8032 & 305.9540 \\ 313.2217 & 315.7881 & 321.8714 \\ 323.2267 & 327.8328 & 328.9494 \\ 331.2253 & 333.9069 & 339.9871 \\ 344.6321 & 351.0011 & 357.7868 \\ 360.8333 & 371.4433 & 385.7135 \\ 386.5711 & 398.0669 & 411.0275 \\ 442.4506 & 443.7680 & 465.8184 \\ 476.8829 & 485.8319 & 493.8106 \\ 499.1725 & 511.8995 & 520.3667 \\ 548.2285 & 553.0482 & 554.6740 \\ 557.7435 & 566.0357 & 571.6716 \\ 584.8754 & 591.0599 & 594.7072 \\ 598.5038 & 601.2328 & 604.0612 \\ 605.2442 & 612.8107 & 623.5086 \\ 629.3558 & 638.1621 & 638.9870 \\ 641.0391 & 653.0870 & 656.8713 \\ 659.6602 & 672.6519 & 676.5009 \\ 680.2579 & 682.7789 & 686.3583 \\ 689.8721 & 691.7823 & 693.8482 \\ 700.5601 & 703.4161 & 704.5847 \\ 708.7552 & 710.3523 & 711.8953 \\ 712.6567 & 714.3714 & 715.7242 \\ 716.8932 & 717.2708 & 720.9018 \\ 726.0485 & 726.5178 & 731.9378 \\ 732.4702 & 737.6995 & 739.4989 \\ 744.1043 & 744.8617 & 749.1230 \\ 752.2612 & 754.8276 & 755.5084 \\ 760.3261 & 771.3497 & 783.5776 \\ 787.4039 & 799.6142 & 804.7781 \\ 813.2226 & 828.0106 & 856.6408\end{array}$




$\begin{array}{lll}861.5644 & 861.8239 & 866.5971 \\ 868.0072 & 873.0374 & 876.8685 \\ 878.6452 & 886.9329 & 891.1598 \\ 897.9523 & 898.9260 & 907.8667 \\ 909.2802 & 911.7023 & 915.6693 \\ 916.6891 & 920.1156 & 922.8976 \\ 924.3361 & 926.1648 & 928.4787 \\ 932.3762 & 936.1712 & 937.2590 \\ 940.2663 & 950.3740 & 963.9071 \\ 968.1625 & 974.3181 & 975.6468 \\ 980.6665 & 983.1229 & 989.3831 \\ 991.5578 & 992.3217 & 994.2674 \\ 998.2213 & 1000.0778 & 1002.7554 \\ 1007.4112 & 1008.7520 & 1010.8799 \\ 1013.8609 & 1016.4875 & 1020.4524 \\ 1023.0354 & 1024.4173 & 1026.1089 \\ 1028.3093 & 1030.0361 & 1030.4584 \\ 1034.7110 & 1040.5265 & 1042.7369 \\ 1044.9206 & 1046.8009 & 1052.6914 \\ 1055.5143 & 1058.5087 & 1065.5798 \\ 1073.7279 & 1076.4384 & 1078.5464 \\ 1087.3529 & 1087.7521 & 1088.5235 \\ 1094.6527 & 1096.7756 & 1101.2136 \\ 1107.4906 & 1112.7471 & 1115.2679 \\ 1117.6974 & 1121.0508 & 1122.5665 \\ 1125.1424 & 1135.8839 & 1138.2778 \\ 1141.3316 & 1147.9399 & 1151.5017 \\ 1156.8125 & 1167.1226 & 1167.9680 \\ 1173.9059 & 1174.3171 & 1177.8173 \\ 1178.5933 & 1179.1387 & 1183.0807 \\ 1188.5578 & 1193.8965 & 1199.6642 \\ 1207.5836 & 1217.6841 & 1222.7281 \\ 1241.7689 & 1244.1798 & 1251.4171 \\ 1267.4155 & 1270.8064 & 1277.3206 \\ 1284.6762 & 1289.2081 & 1318.5891 \\ 1322.4746 & 1330.1589 & 1330.9316 \\ 1334.3425 & 1347.2192 & 1349.8695 \\ 1361.4408 & 1374.5962 & 1384.6154 \\ 1389.0220 & 1394.3757 & 1396.4975 \\ 1401.5376 & 1402.7020 & 1405.8966 \\ 1414.2594 & 1420.4295 & 1420.8478 \\ 1427.9645 & 1428.9602 & 1439.5826 \\ 1443.5016 & 1446.6242 & 1451.3175 \\ 1459.0318 & 1463.7091 & 1467.8996 \\ 1474.5611 & 1478.7566 & 1482.2786 \\ 1482.6654 & 1484.1870 & 1488.8259 \\ 1491.8468 & 1496.7367 & 1499.4866 \\ 1503.3153 & 1507.5683 & 1511.5955 \\ 1513.2395 & 1514.2255 & 1520.1018 \\ 1523.3699 & 1523.6380 & 1525.0880 \\ 1527.3014 & 1527.7959 & 1533.5602 \\ 1535.6099 & 1537.9731 & 1541.9603 \\ 1546.7924 & 1549.0831 & 1550.9397\end{array}$




$\begin{array}{lll}1554.4780 & 1557.5790 & 1561.8507 \\ 1565.8289 & 1567.8725 & 1568.9236 \\ 1573.9972 & 1579.5881 & 1580.2376 \\ 1584.0476 & 1584.8522 & 1586.2247 \\ 1589.9893 & 1592.3546 & 1602.4736 \\ 1610.0989 & 1615.9111 & 1618.5214 \\ 1643.2350 & 1649.8115 & 1651.0870 \\ 1655.7649 & 1659.6714 & 1660.7950 \\ 1672.2286 & 1673.7724 & 1678.9036 \\ 1690.6448 & 1695.0939 & 1702.7062 \\ 1707.1976 & 1717.6129 & 1721.6064 \\ 1724.5142 & 1726.1268 & 1728.2765 \\ 1752.3000 & 1778.0450 & 1802.7458 \\ 1809.1126 & 1811.4742 & 1831.2211 \\ 1834.3043 & 1873.7807 & 1894.9906 \\ 1900.3907 & 2856.4553 & 9813.9982\end{array}$

3.2 Calcite-Super-Molecule, Optimized Geometry (B3LYP/6-31G)
C $\quad-0.0010620 \quad-0.0345180 \quad-0.0213170$
$\begin{array}{llll}\text { O } & 0.6341650 & 1.1120080 & -0.0415350\end{array}$
O $\quad 0.6778980 \quad-1.1520560 \quad-0.0105030$
O $\quad-1.3112390 \quad-0.0488020 \quad-0.0024910$
$\begin{array}{llll}\mathrm{O} & 1.8742720 & 2.7655380 & 2.6816240\end{array}$
Ca $\quad 0.0666880 \quad-2.9601890 \quad-1.4212820$
$\begin{array}{llll}\text { Ca } & -2.6012710 & 1.4206200 & -1.4117340\end{array}$
Ca $\quad-2.5088370 \quad-1.5779770 \quad 1.4098120$
$\begin{array}{llll}\text { Ca } & 2.5194490 & 1.5405110 & -1.4163470\end{array}$
$\begin{array}{llll}\mathrm{Ca} & 2.6070620 & -1.3779700 & 1.4125080\end{array}$
$\begin{array}{llll}\mathrm{Ca} & -0.0684220 & 2.8427010 & 1.3890420\end{array}$
$\begin{array}{llll}\text { O } & -0.8743090 & 6.9317120 & 2.8999830\end{array}$
$\begin{array}{llll}\text { O } & -5.7901390 & 3.8923940 & -2.8632660\end{array}$
$\begin{array}{llll}\text { O } & 6.3273920 & 3.0400760 & -2.9331610\end{array}$
O $\quad-0.3998110 \quad-6.9141250 \quad-2.7168560$
$\begin{array}{llll}\text { C } & 2.3802290 & 4.4533240 & 0.0047090\end{array}$
$\begin{array}{llll}\mathrm{O} & 1.1963000 & 2.9626160 & -2.8880950\end{array}$
O $4.2924020 \quad 4.1669090 \quad-2.6324190$
$\begin{array}{llll}\mathrm{O} & 2.9919440 & 5.5943810 & 0.0065230\end{array}$
O $\quad 1.1055490 \quad 5.8665720 \quad 2.8978280$
$\begin{array}{llll}\text { C } & 5.0412730 & 0.1622620 & -0.0030110\end{array}$
$\begin{array}{llll}\text { O } & 3.8573440 & -1.3284460 & -2.8958150\end{array}$
$\begin{array}{llll}\text { O } & 4.3372380 & 1.9397870 & -2.8903890\end{array}$
$\begin{array}{llll}\text { C } & 4.9489520 & 3.0808450 & -2.8885750\end{array}$
$\begin{array}{llll}\text { O } & 3.0625580 & 3.3530350 & 0.0027300\end{array}$
$\begin{array}{llll}\text { O } & 5.6529870 & 1.3033200 & -0.0011970\end{array}$
$\begin{array}{llll}\mathrm{O} & 3.7665930 & 1.5755110 & 2.8901080\end{array}$
$\begin{array}{llll}\text { O } & 5.7236020 & -0.9380260 & -0.0049900\end{array}$
$\begin{array}{llll}\text { O } & 6.4276370 & -2.7155510 & 2.8823890\end{array}$
$\begin{array}{llll}\text { C } & -2.6664670 & 4.2943250 & 0.0053550\end{array}$
$\begin{array}{llll}\mathrm{O} & -3.8503960 & 2.8036170 & -2.8874500\end{array}$
$\begin{array}{llll}\mathrm{O} & -0.7800730 & 4.0221340 & -2.8859500\end{array}$
$\begin{array}{llll}\mathrm{O} & -2.0547520 & 5.4353820 & 0.0071680\end{array}$
$\begin{array}{llll}\mathrm{O} & -1.1893520 & -1.4874440 & -2.8951690\end{array}$
$\begin{array}{llll}\text { O } & 1.8809710 & -0.2689270 & -2.8936700\end{array}$ 


$\begin{array}{llll}\mathrm{O} & -0.7094580 & 1.7807880 & -2.8897440 \\ \mathrm{C} & -0.0977440 & 2.9218460 & -2.8879300 \\ \mathrm{O} & 1.0861850 & 4.4125540 & 0.0048750 \\ \mathrm{O} & -1.9841380 & 3.1940370 & 0.0033750 \\ \mathrm{O} & -1.2801030 & 1.4165120 & 2.8907540 \\ \mathrm{O} & -0.8002090 & 4.6847450 & 2.8961790 \\ \mathrm{C} & -0.1884940 & 5.8258020 & 2.8979930 \\ \mathrm{C} & 2.6556210 & -4.2877980 & -0.0100850 \\ \mathrm{O} & 1.4716920 & -5.7785060 & -2.9028890 \\ \mathrm{O} & 1.9515860 & -2.5102730 & -2.8974630 \\ \mathrm{C} & 2.5633000 & -1.3692150 & -2.8956490 \\ \mathrm{O} & 3.7472290 & 0.1214930 & -0.0028450 \\ \mathrm{O} & 3.2673350 & -3.1467400 & -0.0082710 \\ \mathrm{O} & 1.3809410 & -2.8745500 & 2.8830340 \\ \mathrm{O} & 4.4512640 & -1.6560320 & 2.8845330 \\ \mathrm{O} & 1.8608350 & 0.3936830 & 2.8884600 \\ \mathrm{C} & 2.4725490 & 1.5347410 & 2.8902740 \\ \mathrm{O} & 3.3379500 & -5.3880860 & -0.0120640 \\ \mathrm{O} & 4.5218790 & -3.8973780 & 2.8807400 \\ \mathrm{C} & 5.1335930 & -2.7563200 & 2.8825540 \\ \mathrm{C} & -5.052190 & -0.1557350 & -0.0017200 \\ \mathrm{O} & -3.1657250 & -0.4279260 & -2.8930240 \\ \mathrm{O} & -5.7561540 & 1.6217900 & -2.8890980 \\ \mathrm{C} & -5.1444400 & 2.7628470 & -2.8872840 \\ \mathrm{O} & -3.9605100 & 4.2535550 & 0.0055200 \\ \mathrm{O} & -4.4404040 & 0.9853220 & 0.0000940 \\ \mathrm{O} & -3.2564750 & 2.4760300 & 2.8928990 \\ \mathrm{C} & -2.3910750 & -4.4467970 & -0.0094390 \\ \mathrm{O} & -0.5046810 & -4.7189870 & -2.9007440 \\ \mathrm{O} & -3.0951100 & -2.6692720 & -2.8968180 \\ \mathrm{C} & -2.4833960 & -1.5282140 & -2.8950040 \\ \mathrm{O} & -4.3697900 & -1.2560230 & -0.0036990 \\ \mathrm{O} & -1.7793610 & -3.3057390 & -0.0076250 \\ \mathrm{H} & -3.3757340 & 3.4046030 & -3.4663380 \\ \mathrm{O} & -3.6657550 & -3.0335480 & 2.8836790 \\ \mathrm{O} & -0.5954310 & -1.8150310 & 2.8851790 \\ \mathrm{O} & -3.1858610 & 0.2346840 & 2.8891050 \\ \mathrm{C} & -2.5741460 & 1.3757420 & 2.8909190 \\ \mathrm{C} & 0.1776480 & -5.8192750 & -2.9027230 \\ \mathrm{O} & 1.3615770 & -4.3285670 & -0.0099190 \\ \mathrm{O} & -1.7087460 & -5.5470850 & -0.0114190 \\ \mathrm{O} & -0.5248170 & -4.0563770 & 2.8813860 \\ \mathrm{C} & 0.0868970 & -2.9153190 & 2.8832000 \\ \mathrm{O} & -6.3461630 & -0.1965050 & -0.0015540 \\ \mathrm{O} & -5.6421270 & -1.9740300 & 2.8858240 \\ \mathrm{O} & -3.6851190 & -4.4875660 & -0.0092740 \\ \mathrm{O} & -5.5715130 & -4.2153760 & 2.8820310 \\ \mathrm{H} & -4.9597980 & -3.0743180 & 2.8838450 \\ \mathrm{H} & 1.1238600 & -0.1567940 & -3.4731600 \\ & -0.4273240 & 1.0706030 & -3.4708090 \\ \mathrm{H} & & \\ \mathrm{H} & & \end{array}$




\begin{tabular}{llll}
\hline $\mathrm{H}$ & 3.4384020 & 4.3025850 & -3.0494040 \\
$\mathrm{H}$ & 4.3320050 & -0.7274590 & -3.4747030 \\
$\mathrm{H}$ & 4.6193720 & 1.2296020 & -3.4714540 \\
$\mathrm{H}$ & 1.9463530 & -5.1775190 & -3.4817770 \\
$\mathrm{H}$ & 2.2337200 & -3.2204580 & -3.4785280 \\
$\mathrm{H}$ & 4.2782930 & 1.0056000 & 3.4688700 \\
$\mathrm{H}$ & 4.6882830 & -0.9294900 & 3.4655320 \\
$\mathrm{H}$ & 1.8926410 & -3.4444600 & 3.4617960 \\
$\mathrm{H}$ & 3.7734760 & -4.0569430 & 3.4604220 \\
$\mathrm{H}$ & -3.1540550 & -3.6034590 & 3.4624420 \\
$\mathrm{H}$ & -1.2732200 & -4.2159420 & 3.4610680 \\
$\mathrm{H}$ & -0.7684030 & 0.8466010 & 3.4695160 \\
$\mathrm{H}$ & 1.1124320 & 0.2341180 & 3.4681420 \\
$\mathrm{H}$ & -0.3584130 & -1.0884890 & 3.4661780 \\
$\mathrm{H}$ & 1.6172490 & 5.2966610 & 3.4765900 \\
$\mathrm{H}$ & 2.0272390 & 3.3615710 & 3.4732520 \\
$\mathrm{H}$ & -3.9342640 & 0.0751190 & 3.4687880 \\
$\mathrm{H}$ & -5.4051090 & -1.2474880 & 3.4668230 \\
$\mathrm{H}$ & -1.5486120 & 4.5251790 & 3.4758620 \\
$\mathrm{H}$ & -3.0194570 & 3.2025720 & 3.4738970 \\
$\mathrm{H}$ & 4.6483770 & 4.8296550 & -2.0360550 \\
$\mathrm{H}$ & 3.7403470 & 5.7539470 & -0.5731590 \\
$\mathrm{H}$ & 6.4013910 & 1.4628850 & -0.5808790 \\
$\mathrm{H}$ & 5.4865830 & -1.6645680 & -0.5859890 \\
$\mathrm{H}$ & 4.0157380 & -2.9871750 & -0.5879530 \\
$\mathrm{H}$ & 4.3690430 & -1.8983560 & -2.3170530 \\
$\mathrm{H}$ & -4.6068090 & -1.9825650 & -0.5846980 \\
$\mathrm{H}$ & -4.1968180 & -3.9176560 & -0.5880360 \\
$\mathrm{H}$ & -3.8435140 & -2.8288370 & -2.3171350 \\
$\mathrm{H}$ & -1.9457650 & -6.2736260 & -0.5924170 \\
$\mathrm{H}$ & 3.1009310 & -6.1146280 & -0.5930630 \\
$\mathrm{H}$ & 1.9833910 & -6.3484160 & -2.3241270 \\
$\mathrm{H}$ & -4.4722100 & 4.8234660 & -0.5732420 \\
$\mathrm{H}$ & -6.8578620 & 0.3734060 & -0.5803160 \\
$\mathrm{H}$ & -6.5045570 & 1.4622240 & -2.3094160 \\
$\mathrm{H}$ & -6.8208240 & -0.7974910 & 0.5773340 \\
$\mathrm{H}$ & -6.3992390 & -1.8618970 & 2.3063350 \\
$\mathrm{H}$ & -4.1597810 & -5.0885520 & 0.5696150 \\
$\mathrm{H}$ & 0.8869150 & -4.9295540 & 0.5689690 \\
$\mathrm{H}$ & -5.2893780 & -4.9255610 & 2.3009660 \\
$\mathrm{H}$ & -4.4351720 & 3.6525690 & 0.5844080 \\
$\mathrm{H}$ & -4.7225390 & 1.6955080 & 0.5811590 \\
$\mathrm{H}$ & -4.0135870 & 2.5881640 & 2.3134090 \\
$\mathrm{H}$ & -2.3368870 & 6.1455680 & 0.5882330 \\
$\mathrm{H}$ & 2.7098090 & 6.3045670 & 0.5875880 \\
$\mathrm{H}$ & 1.5802110 & 6.4675580 & 2.3189390 \\
$\mathrm{H}$ & 3.8196690 & 3.2409020 & 0.5822190 \\
$\mathrm{H}$ & 5.3708530 & 2.0135060 & 0.5798680 \\
$\mathrm{H}$ & 4.2412550 & 2.1764970 & 2.3112200 \\
$\mathrm{H}$ & 6.4807130 & -1.0501590 & 0.5745000 \\
\hline
\end{tabular}




$\begin{array}{llll}\mathrm{H} & -0.9516350 & -5.6592180 & 0.5680710 \\ \mathrm{H} & -0.2426820 & -4.7665620 & 2.3003210 \\ \mathrm{H} & -1.3063490 & 5.5949480 & -0.5725140 \\ \mathrm{H} & 0.5744860 & 4.9824650 & -0.5738880 \\ \mathrm{H} & -0.5430540 & 4.7486760 & -2.3049520 \\ \mathrm{H} & -0.2029390 & -7.6716010 & -3.2727990 \\ \mathrm{H} & -0.8787250 & -7.0864230 & -1.9028810 \\ \mathrm{H} & -0.9739050 & -4.5201890 & -3.7143210 \\ \mathrm{H} & -2.9511100 & -3.4377560 & -3.4538600 \\ \mathrm{H} & -3.8653890 & -0.2408890 & -3.5231730 \\ \mathrm{H} & -5.3349520 & 0.9023180 & -3.3650710 \\ \mathrm{H} & 6.9379720 & -3.3160690 & 3.4306000 \\ \mathrm{H} & 6.7094480 & 3.7278750 & -3.4832220 \\ \mathrm{H} & 6.8010860 & 2.4627720 & -2.3298920 \\ \mathrm{H} & -6.3886590 & 3.9353690 & -3.6126170 \\ \mathrm{H} & -5.6690310 & 4.6594240 & -2.2988200 \\ \mathrm{H} & -0.6520820 & 7.4451910 & 3.6800810 \\ \mathrm{H} & -6.3461110 & -4.2982400 & 3.4430410 \\ \mathrm{H} & -1.4439690 & 7.4161540 & 2.2979840\end{array}$

Frequencies (not scaled) C-12( $1^{\text {st }}$ atom $) \mathrm{O} 16\left(2^{\text {nd }}\right.$ atom):

$\begin{array}{lll}-3321.7981 & -1602.5775 & -1352.7928 \\ -1226.6252 & -1204.1930 & -1166.5604 \\ -1136.7778 & -1106.1921 & -1100.8697 \\ -1098.7335 & -1093.7919 & -1091.7590 \\ -1081.8757 & -1079.4406 & -1071.7077 \\ -1069.1894 & -1063.0607 & -1060.7296 \\ -1056.8575 & -1055.5293 & -1053.9390 \\ -1052.0844 & -1045.6011 & -1042.9137 \\ -1040.7907 & -1035.6329 & -1035.0568 \\ -1031.2455 & -1028.8806 & -1025.1590 \\ -1020.7188 & -1019.5218 & -1012.4688 \\ -1006.5072 & -1005.3708 & -1000.5926 \\ -999.7570 & -995.7027 & -992.3508 \\ -989.6121 & -985.1151 & -979.5400 \\ -974.7300 & -971.5528 & -969.1526 \\ -964.2749 & -961.3124 & -958.3096 \\ -952.9169 & -949.6574 & -945.4404 \\ -941.4647 & -933.6505 & -931.0706 \\ -929.5400 & -923.3223 & -918.9698 \\ -917.3621 & -913.4966 & -910.1091 \\ -907.5373 & -904.2211 & -897.7662 \\ -896.0079 & -891.9802 & -890.3001 \\ -885.3306 & -879.1031 & -876.0451 \\ -869.7339 & -865.3447 & -858.4852 \\ -851.2349 & -849.1834 & -846.6720 \\ -842.2338 & -840.4016 & -838.0143 \\ -830.3399 & -827.3898 & -823.9854 \\ -817.2012 & -815.7150 & -810.9299 \\ -805.8690 & -803.7283 & -798.9532 \\ -795.8511 & -793.2904 & -779.8742 \\ -774.0226 & -756.5746 & -748.7377 \\ -716.1114 & -545.3387 & -497.2548 \\ -458.8176 & -449.8208 & -442.4416\end{array}$




$\begin{array}{lll}-439.2412 & -431.7137 & -424.5649 \\ -422.3336 & -419.0236 & -412.0560 \\ -411.0587 & -400.3035 & -391.4256 \\ -384.7515 & -381.5476 & -374.6206 \\ -373.6481 & -360.7179 & -355.4887 \\ -349.9711 & -347.8635 & -346.8953 \\ -343.8749 & -335.4791 & -327.8971 \\ -318.9477 & -311.9006 & -288.5289 \\ -263.9737 & -255.8104 & -253.6853 \\ -249.4138 & -248.1895 & -244.8034 \\ -242.5575 & -240.1254 & -234.6246 \\ -231.7996 & -222.6442 & -221.2681 \\ -209.5141 & -208.2501 & -201.5911 \\ -195.6224 & -193.7509 & -188.2179 \\ -184.6079 & -179.8252 & -165.0619 \\ -159.5234 & -148.4687 & -138.4023 \\ -131.3963 & -120.5305 & -108.7541 \\ -69.9491 & 25.4616 & 42.1790 \\ 48.5633 & 51.8386 & 56.3258 \\ 59.4821 & 62.9190 & 63.9809 \\ 68.2523 & 71.3371 & 74.0961 \\ 77.1275 & 80.4135 & 81.6972 \\ 84.3243 & 85.5870 & 87.8438 \\ 90.4406 & 91.0501 & 93.5600 \\ 95.2779 & 98.2202 & 100.6320 \\ 103.0211 & 106.4775 & 107.8649 \\ 108.8046 & 111.0974 & 111.9197 \\ 115.7353 & 117.7640 & 118.6368 \\ 122.7985 & 126.0074 & 128.7813 \\ 132.1446 & 134.1036 & 136.4970 \\ 139.4374 & 142.2917 & 153.1528 \\ 157.5177 & 158.5537 & 162.6642 \\ 163.4989 & 166.0476 & 170.0474 \\ 172.6546 & 175.5307 & 176.0059 \\ 178.7284 & 182.5771 & 185.9566 \\ 188.6307 & 189.3238 & 194.3494 \\ 200.8869 & 215.3192 & 216.7253 \\ 222.3991 & 226.6671 & 231.6342 \\ 234.8286 & 239.4254 & 244.1828 \\ 247.5278 & 255.6333 & 257.2524 \\ 260.3205 & 260.9646 & 264.2550 \\ 267.6146 & 279.9726 & 295.3382 \\ 296.3675 & 300.5932 & 302.8462 \\ 305.5315 & 310.2740 & 314.5210 \\ 318.6319 & 319.7185 & 324.2256 \\ 337.8686 & 342.2271 & 342.6678 \\ 349.3961 & 357.6020 & 362.3435 \\ 364.0540 & 370.4161 & 371.6702 \\ 378.2354 & 402.5655 & 414.8030 \\ 419.5829 & 424.4624 & 427.9865 \\ 430.6962 & 436.6242 & 437.8133 \\ 442.7379 & 447.7770 & 452.6603 \\ 455.3237 & 465.4020 & 470.7657\end{array}$




$\begin{array}{lll}484.7722 & 563.8819 & 628.6801 \\ 663.9733 & 671.8361 & 684.5855 \\ 689.6440 & 693.7125 & 697.7559 \\ 700.6274 & 704.0314 & 714.1949 \\ 716.7313 & 744.0702 & 752.9625 \\ 756.8960 & 767.7440 & 770.0629 \\ 777.8317 & 784.3216 & 788.2642 \\ 795.8568 & 798.2599 & 806.0026 \\ 812.7866 & 813.7266 & 819.5918 \\ 833.3534 & 840.3056 & 844.6999 \\ 875.2967 & 889.0791 & 898.5207 \\ 907.4872 & 921.2449 & 928.7319 \\ 942.3337 & 948.1960 & 952.8356 \\ 958.4759 & 964.3750 & 967.7808 \\ 973.1892 & 984.7151 & 989.1694 \\ 993.4569 & 995.5236 & 996.1605 \\ 999.9932 & 1006.6296 & 1009.9217 \\ 1011.3158 & 1013.0661 & 1021.7786 \\ 1024.4843 & 1035.2010 & 1040.8590 \\ 1043.3146 & 1046.4110 & 1047.1505 \\ 1048.6717 & 1058.7031 & 1059.5836 \\ 1062.4299 & 1065.3535 & 1073.1516 \\ 1074.5830 & 1079.7370 & 1083.7990 \\ 1095.8316 & 1107.8786 & 1127.3569 \\ 1137.6551 & 1172.2620 & 1175.7260 \\ 1197.5397 & 1205.1112 & 1214.3377 \\ 1218.9298 & 1219.4882 & 1232.6001 \\ 1245.5102 & 1251.1327 & 1253.9105 \\ 1258.0257 & 1262.3654 & 1273.0381 \\ 1280.3512 & 1284.2864 & 1289.9420 \\ 1293.8011 & 1298.4657 & 1303.7511 \\ 1307.7004 & 1318.2124 & 1354.5140 \\ 1376.4093 & 1387.3236 & 1442.4731 \\ 1453.3676 & 1476.2010 & 1483.6373 \\ 1489.6288 & 1517.8164 & 1548.9343 \\ 1563.1894 & 1592.4113 & 1596.6582 \\ 1624.0599 & 1640.5034 & 1641.9212 \\ 1657.8908 & 1664.5168 & 1674.8514 \\ 1676.4961 & 1679.3358 & 1692.0463 \\ 1692.6146 & 1700.1386 & 1707.9762 \\ 1717.4968 & 1735.3633 & 1739.1869 \\ 1740.8271 & 1744.1306 & 1748.1217 \\ 1749.3857 & 1756.0504 & 1763.1437 \\ 1767.2306 & 1769.3772 & 1775.1741 \\ 1787.5207 & 1788.5219 & 1791.3144 \\ 1802.8350 & 1804.6541 & 1863.9249 \\ 1895.7743 & 2320.9356 & 2618.0299 \\ 2717.3327 & 2731.5892 & 2790.0171 \\ 2894.3691 & 2926.2515 & 2937.3797 \\ 2964.3831 & 2995.4997 & 3001.5027 \\ 3029.5976 & 3041.7402 & 3067.4680 \\ 3078.5990 & 3081.5578 & 3094.8743 \\ 3097.5822 & 3106.5933 & 3112.2715\end{array}$




\begin{tabular}{|c|c|c|}
\hline 3124.1373 & 3139.4996 & 3143.8235 \\
\hline 3156.5196 & 3170.4048 & 3181.6290 \\
\hline 3190.5344 & 3208.8917 & 3211.7270 \\
\hline 3220.6028 & 3221.9582 & 3230.6192 \\
\hline 3237.4886 & 3248.4949 & 3249.2595 \\
\hline 3255.5952 & 3256.6788 & 3264.3165 \\
\hline 3269.0521 & 3276.8967 & 3278.8036 \\
\hline 3281.4508 & 3284.8670 & 3292.7586 \\
\hline 3296.8925 & 3301.8276 & 3306.2414 \\
\hline 3314.0576 & 3317.8605 & 3322.7167 \\
\hline 3325.8866 & 3326.5315 & 3329.3634 \\
\hline 3332.9838 & 3337.2468 & 3339.5104 \\
\hline 3342.8975 & 3346.6696 & 3350.7381 \\
\hline 3360.4953 & 3377.5613 & 3382.6810 \\
\hline 3386.7782 & 3399.1933 & 3399.5642 \\
\hline 3408.2199 & 3410.7488 & 3419.6224 \\
\hline 3423.2761 & 3436.3044 & 3459.9827 \\
\hline 3469.1146 & 3473.6331 & 3494.5962 \\
\hline 3507.4135 & 3512.0593 & 3561.4252 \\
\hline \multicolumn{3}{|c|}{ Frequencies (not scaled) C-12( $1^{\text {st }}$ atom $) \mathrm{O} 18\left(2^{\text {nd }}\right.$ atom $)$} \\
\hline-3321.7957 & -1602.5741 & -1352.7868 \\
\hline-1226.6245 & -1204.1908 & -1166.5596 \\
\hline-1136.7773 & -1106.1916 & -1100.8695 \\
\hline-1098.7332 & -1093.7918 & -1091.7590 \\
\hline-1081.8757 & -1079.4402 & -1071.7076 \\
\hline-1069.1893 & -1063.0606 & -1060.7295 \\
\hline-1056.8574 & -1055.5292 & -1053.9387 \\
\hline-1052.0834 & -1045.6010 & -1042.9132 \\
\hline-1040.7902 & -1035.6329 & -1035.0565 \\
\hline-1031.2453 & -1028.8804 & -1025.1589 \\
\hline-1020.7179 & -1019.5211 & -1012.4686 \\
\hline-1006.5069 & -1005.3706 & -1000.5924 \\
\hline-999.7567 & -995.7023 & -992.3505 \\
\hline-989.6120 & -985.1151 & -979.5398 \\
\hline-974.7294 & -971.5524 & -969.1522 \\
\hline-964.2747 & -961.3123 & -958.3095 \\
\hline-952.9161 & -949.6572 & -945.4404 \\
\hline-941.4644 & -933.6501 & -931.0704 \\
\hline-929.5398 & -923.3216 & -918.9695 \\
\hline-917.3621 & -913.4962 & -910.1078 \\
\hline-907.5367 & -904.2208 & -897.7661 \\
\hline-896.0071 & -891.9800 & -890.3000 \\
\hline-885.3274 & -879.0970 & -876.0449 \\
\hline-869.7328 & -865.3413 & -858.4848 \\
\hline-851.2338 & -849.1825 & -846.6714 \\
\hline-842.2331 & -840.4007 & -838.0143 \\
\hline-830.3398 & -827.3894 & -823.9853 \\
\hline-817.2001 & -815.7140 & -810.9298 \\
\hline-805.8684 & -803.7278 & -798.9528 \\
\hline-795.8510 & -793.2902 & -779.8742 \\
\hline-774.0226 & -756.5621 & -748.7376 \\
\hline-716.1113 & -545.3386 & -497.2542 \\
\hline-458.8174 & -449.8200 & -442.4406 \\
\hline
\end{tabular}




$\begin{array}{lll}-439.2411 & -431.7129 & -424.5647 \\ -422.3336 & -419.0230 & -412.0560 \\ -411.0584 & -400.3031 & -391.4246 \\ -384.7508 & -381.5474 & -374.6189 \\ -373.6475 & -360.7160 & -355.4885 \\ -349.9692 & -347.8621 & -346.8953 \\ -343.8746 & -335.4790 & -327.8962 \\ -318.9474 & -311.9006 & -288.5280 \\ -263.9728 & -255.8093 & -253.6838 \\ -249.4069 & -248.1887 & -244.8031 \\ -242.5529 & -240.1089 & -234.6215 \\ -231.7947 & -222.6396 & -221.2676 \\ -209.5139 & -208.2485 & -201.5891 \\ -195.6190 & -193.7479 & -188.2069 \\ -184.6042 & -179.8217 & -165.0567 \\ -159.5190 & -148.4684 & -138.4007 \\ -131.3910 & -120.5271 & -108.7515 \\ -69.9374 & 25.4607 & 42.1788 \\ 48.5619 & 51.8308 & 56.3238 \\ 59.4737 & 62.8885 & 63.9117 \\ 68.2379 & 71.3258 & 74.0582 \\ 77.1203 & 80.3942 & 81.6377 \\ 84.2848 & 85.5621 & 87.8182 \\ 90.3677 & 91.0055 & 93.5581 \\ 95.2582 & 98.2036 & 100.6220 \\ 102.9421 & 106.4539 & 107.8457 \\ 108.7767 & 111.0645 & 111.8200 \\ 115.7314 & 117.7114 & 118.6200 \\ 122.7977 & 125.7520 & 128.5926 \\ 132.0836 & 133.7806 & 135.6468 \\ 139.2032 & 142.1997 & 153.0352 \\ 157.3905 & 158.2521 & 162.6231 \\ 163.0614 & 165.6626 & 169.9807 \\ 172.6271 & 175.1981 & 175.7857 \\ 177.9870 & 182.1700 & 185.5230 \\ 188.6126 & 189.1757 & 192.9495 \\ 200.3944 & 214.0832 & 216.6096 \\ 222.1073 & 226.5529 & 231.5741 \\ 234.7845 & 239.3993 & 244.1112 \\ 247.5021 & 255.5832 & 257.2368 \\ 260.2135 & 260.6141 & 264.2478 \\ 267.5951 & 279.8508 & 295.2704 \\ 296.3308 & 300.3672 & 302.5703 \\ 305.1930 & 309.9956 & 314.0445 \\ 318.1653 & 319.5229 & 324.0170 \\ 335.4258 & 341.2471 & 342.4388 \\ 349.2450 & 357.2644 & 361.9057 \\ 363.8756 & 369.6360 & 370.6129 \\ 375.3425 & 402.4782 & 414.6997 \\ 419.5748 & 424.4481 & 427.9798 \\ 430.6889 & 436.6149 & 437.8040 \\ 442.7305 & 447.7718 & 452.6484 \\ 455.3091 & 465.4007 & 470.7640\end{array}$




$\begin{array}{lll}484.7671 & 563.8722 & 628.6764 \\ 663.9311 & 671.8254 & 679.7676 \\ 683.4722 & 684.9556 & 694.1118 \\ 700.4850 & 702.7159 & 714.1360 \\ 716.7286 & 744.0684 & 752.9593 \\ 756.8943 & 767.7408 & 770.0594 \\ 777.8261 & 784.3134 & 788.2634 \\ 795.8562 & 798.2598 & 805.9832 \\ 810.9502 & 813.6984 & 818.0083 \\ 833.3223 & 840.2996 & 844.6993 \\ 875.2940 & 889.0735 & 898.5186 \\ 907.4758 & 921.2317 & 928.7099 \\ 942.3181 & 948.1671 & 952.8121 \\ 958.4694 & 964.3659 & 967.7725 \\ 973.1819 & 984.7130 & 989.1611 \\ 993.4536 & 995.5233 & 996.1601 \\ 999.9931 & 1006.6291 & 1009.9215 \\ 1011.3157 & 1013.0658 & 1021.7745 \\ 1024.4830 & 1035.0618 & 1038.3481 \\ 1040.8661 & 1043.3978 & 1046.4146 \\ 1047.1503 & 1048.6915 & 1059.5502 \\ 1062.4096 & 1065.3521 & 1073.1441 \\ 1074.5811 & 1079.7369 & 1083.7990 \\ 1095.8316 & 1107.8785 & 1127.3565 \\ 1137.6549 & 1172.2616 & 1175.7245 \\ 1197.5382 & 1205.1100 & 1214.3377 \\ 1218.9297 & 1219.4882 & 1232.5995 \\ 1245.5098 & 1251.1326 & 1253.9105 \\ 1258.0256 & 1262.3647 & 1273.0372 \\ 1280.3506 & 1284.2863 & 1289.9409 \\ 1293.8010 & 1298.4656 & 1303.7511 \\ 1307.7003 & 1318.2121 & 1354.5124 \\ 1376.4088 & 1387.3234 & 1442.4730 \\ 1453.3386 & 1464.0417 & 1483.5509 \\ 1487.8969 & 1517.7991 & 1548.8397 \\ 1563.1848 & 1592.4095 & 1596.6579 \\ 1624.0439 & 1640.4925 & 1641.8920 \\ 1657.8900 & 1664.5081 & 1674.8413 \\ 1676.4943 & 1679.3344 & 1692.0301 \\ 1692.5957 & 1700.1367 & 1707.9675 \\ 1717.4959 & 1735.3631 & 1739.1864 \\ 1740.8261 & 1744.1293 & 1748.1213 \\ 1749.3853 & 1756.0447 & 1763.1393 \\ 1767.2290 & 1769.3771 & 1775.1737 \\ 1787.5204 & 1788.5214 & 1791.3140 \\ 1802.8348 & 1804.6536 & 1863.9248 \\ 1895.7707 & 2320.9329 & 2618.0292 \\ 2717.3318 & 2731.5889 & 2790.0166 \\ 2894.3680 & 2926.2506 & 2937.3795 \\ 2964.3831 & 2995.4988 & 3001.5026 \\ 3029.5973 & 3041.7401 & 3067.4680 \\ 3078.5988 & 3081.5577 & 3094.8742 \\ 3097.5822 & 3106.5932 & 3112.2715\end{array}$




$\begin{array}{lll}3124.1371 & 3139.4995 & 3143.8234 \\ 3156.5195 & 3170.4047 & 3181.6284 \\ 3190.5343 & 3208.8915 & 3211.7270 \\ 3220.6027 & 3221.9582 & 3230.6192 \\ 3237.4886 & 3248.4949 & 3249.2594 \\ 3255.5951 & 3256.6788 & 3264.3164 \\ 3269.0520 & 3276.8966 & 3278.8036 \\ 3281.4508 & 3284.8669 & 3292.7586 \\ 3296.8925 & 3301.8274 & 3306.2412 \\ 3314.0575 & 3317.8605 & 3322.7167 \\ 3325.8866 & 3326.5315 & 3329.3633 \\ 3332.9837 & 3337.2467 & 3339.5104 \\ 3342.8974 & 3346.6695 & 3350.7381 \\ 3360.4952 & 3377.5613 & 3382.6809 \\ 3386.7781 & 3399.1932 & 3399.5642 \\ 3408.2199 & 3410.7488 & 3419.6224 \\ 3423.2761 & 3436.3044 & 3459.9827 \\ 3469.1145 & 3473.6331 & 3494.5962 \\ 3507.4135 & 3512.0593 & 3561.4252\end{array}$

Frequencies (not scaled) C-13( $1^{\text {st }}$ atom $) \mathrm{O} 16\left(2^{\text {nd }}\right.$ atom):

$\begin{array}{lll}-3321.7963 & -1602.5771 & -1352.7870\end{array}$

$\begin{array}{lll}-1226.6242 & -1204.1899 & -1166.5599\end{array}$

$\begin{array}{lll}-1136.7769 & -1106.1913 & -1100.8692\end{array}$

$\begin{array}{lll}-1098.7331 & -1093.7919 & -1091.7590\end{array}$

$\begin{array}{lll}-1081.8757 & -1079.4403 & -1071.7077\end{array}$

$\begin{array}{lll}-1069.1893 & -1063.0606 & -1060.7295\end{array}$

$\begin{array}{lll}-1056.8575 & -1055.5292 & -1053.9388\end{array}$

$\begin{array}{lll}-1052.0842 & -1045.6011 & -1042.9134\end{array}$

$\begin{array}{lll}-1040.7906 & -1035.6328 & -1035.0566\end{array}$

$\begin{array}{lll}-1031.2454 & -1028.8803 & -1025.1589\end{array}$

$\begin{array}{lll}-1020.7186 & -1019.5217 & -1012.4682\end{array}$

$\begin{array}{lll}-1006.5066 & -1005.3707 & -1000.5922\end{array}$

$\begin{array}{lll}-999.7568 & -995.7022 & -992.3506\end{array}$

$\begin{array}{lll}-989.6120 & -985.1147 & -979.5398\end{array}$

$\begin{array}{lll}-974.7298 & -971.5528 & -969.1524\end{array}$

$\begin{array}{lll}-964.2748 & -961.3123 & -958.3096\end{array}$

$\begin{array}{lll}-952.9169 & -949.6573 & -945.4404\end{array}$

$\begin{array}{lll}-941.4645 & -933.6504 & -931.0705\end{array}$

$\begin{array}{lll}-929.5399 & -923.3223 & -918.9698\end{array}$

$\begin{array}{lll}-917.3620 & -913.4966 & -910.1085\end{array}$

$\begin{array}{lll}-907.5372 & -904.2210 & -897.7661\end{array}$

$\begin{array}{lll}-896.0077 & -891.9801 & -890.2998\end{array}$

$\begin{array}{lll}-885.3304 & -879.1021 & -876.0450\end{array}$

$\begin{array}{lll}-869.7330 & -865.3445 & -858.4846\end{array}$

$\begin{array}{lll}-851.2341 & -849.1831 & -846.6715\end{array}$

$\begin{array}{lll}-842.2333 & -840.4011 & -838.0140\end{array}$

$\begin{array}{lll}-830.3399 & -827.3893 & -823.9854\end{array}$

$\begin{array}{lll}-817.2011 & -815.7148 & -810.9297\end{array}$

$\begin{array}{lll}-805.8683 & -803.7282 & -798.9531\end{array}$

$\begin{array}{lll}-795.8510 & -793.2901 & -779.8742\end{array}$

$\begin{array}{lll}-774.0226 & -756.5729 & -748.7377\end{array}$

$\begin{array}{lll}-716.1114 & -545.3387 & -497.2548\end{array}$

$\begin{array}{lll}-458.8176 & -449.8208 & -442.4416\end{array}$ 


$\begin{array}{lll}-439.2412 & -431.7137 & -424.5649 \\ -422.3336 & -419.0236 & -412.0560 \\ -411.0587 & -400.3035 & -391.4256 \\ -384.7515 & -381.5475 & -374.6204 \\ -373.6481 & -360.7178 & -355.4885 \\ -349.9709 & -347.8634 & -346.8953 \\ -343.8748 & -335.4791 & -327.8969 \\ -318.9476 & -311.9006 & -288.5288 \\ -263.9734 & -255.8099 & -253.6848 \\ -249.4112 & -248.1882 & -244.8020 \\ -242.5559 & -240.1231 & -234.6231 \\ -231.7986 & -222.6435 & -221.2679 \\ -209.5141 & -208.2497 & -201.5903 \\ -195.6214 & -193.7501 & -188.2146 \\ -184.6062 & -179.8248 & -165.0612 \\ -159.5229 & -148.4685 & -138.4017 \\ -131.3954 & -120.5298 & -108.7541 \\ -69.9485 & 25.4609 & 42.1786 \\ 48.5631 & 51.8358 & 56.3233 \\ 59.4803 & 62.9135 & 63.9693 \\ 68.2427 & 71.3309 & 74.0798 \\ 77.1231 & 80.4033 & 81.6883 \\ 84.3086 & 85.5773 & 87.8417 \\ 90.4262 & 91.0498 & 93.5578 \\ 95.2734 & 98.2189 & 100.6317 \\ 103.0117 & 106.4676 & 107.8644 \\ 108.8041 & 111.0932 & 111.9134 \\ 115.7344 & 117.7518 & 118.6316 \\ 122.7976 & 125.9754 & 128.7601 \\ 132.1350 & 134.0472 & 136.2854 \\ 139.3889 & 142.2612 & 153.0857 \\ 157.4557 & 158.4196 & 162.6563 \\ 163.3487 & 165.9553 & 170.0170 \\ 172.6398 & 175.4178 & 175.9339 \\ 178.6141 & 182.5467 & 185.9454 \\ 188.6084 & 189.2293 & 193.9456 \\ 200.6379 & 215.1736 & 216.5707 \\ 222.3359 & 226.6329 & 231.6231 \\ 234.8190 & 239.4193 & 244.1807 \\ 247.5187 & 255.6237 & 257.2522 \\ 260.3061 & 260.9171 & 264.2503 \\ 267.6059 & 279.8069 & 295.3221 \\ 296.3412 & 300.5302 & 302.7627 \\ 305.2594 & 310.0764 & 314.4810 \\ 318.5869 & 319.6539 & 324.1741 \\ 337.6478 & 342.0435 & 342.6073 \\ 349.2406 & 357.4101 & 362.2449 \\ 364.0119 & 370.3219 & 371.5535 \\ 377.6483 & 402.5485 & 414.7694 \\ 419.5798 & 424.4603 & 427.9834 \\ 430.6935 & 436.6240 & 437.8079 \\ 442.7354 & 447.7715 & 452.6567 \\ 455.3216 & 465.4010 & 470.7641\end{array}$




$\begin{array}{lll}484.7719 & 563.8720 & 628.6479 \\ 663.8964 & 671.8291 & 684.4130 \\ 687.7523 & 693.5615 & 696.2729 \\ 700.5724 & 703.6917 & 714.1844 \\ 716.7141 & 744.0575 & 752.9619 \\ 756.8914 & 767.7182 & 770.0547 \\ 777.8273 & 784.3203 & 788.2628 \\ 792.1750 & 795.8580 & 798.2635 \\ 806.0660 & 813.6827 & 816.1314 \\ 833.2854 & 840.2927 & 844.6993 \\ 875.2703 & 889.0433 & 898.5051 \\ 907.4643 & 921.2311 & 928.6713 \\ 942.3301 & 948.1802 & 952.8259 \\ 958.4746 & 964.3728 & 967.7746 \\ 973.1873 & 984.7119 & 989.1598 \\ 993.4529 & 995.5210 & 996.1536 \\ 999.9928 & 1006.6273 & 1009.9213 \\ 1011.3158 & 1013.0656 & 1021.7783 \\ 1024.4831 & 1035.1949 & 1040.8523 \\ 1043.3095 & 1046.4099 & 1047.1466 \\ 1048.6681 & 1058.6995 & 1059.5809 \\ 1062.4289 & 1065.3531 & 1073.1496 \\ 1074.5788 & 1079.7369 & 1083.7989 \\ 1095.8314 & 1107.8781 & 1127.3551 \\ 1137.6547 & 1172.2593 & 1175.7228 \\ 1197.5372 & 1205.1072 & 1214.3377 \\ 1218.9293 & 1219.4882 & 1232.5969 \\ 1245.5078 & 1251.1316 & 1253.9095 \\ 1258.0254 & 1262.3630 & 1273.0341 \\ 1280.3482 & 1284.2858 & 1289.9325 \\ 1293.7961 & 1298.4646 & 1303.7510 \\ 1307.7001 & 1318.2112 & 1354.5072 \\ 1376.4058 & 1387.3227 & 1435.0493 \\ 1442.4721 & 1446.8014 & 1453.4289 \\ 1484.5336 & 1517.7850 & 1548.7448 \\ 1563.1764 & 1592.4065 & 1596.6572 \\ 1623.9547 & 1640.4483 & 1641.8248 \\ 1657.8894 & 1664.3841 & 1674.8287 \\ 1676.4920 & 1679.3327 & 1691.9893 \\ 1692.5110 & 1700.1230 & 1707.9476 \\ 1717.4938 & 1735.3629 & 1739.1857 \\ 1740.8237 & 1744.1197 & 1748.1209 \\ 1749.3837 & 1756.0317 & 1763.1278 \\ 1767.2214 & 1769.3760 & 1775.1726 \\ 1787.5194 & 1788.5190 & 1791.3133 \\ 1802.8333 & 1804.6530 & 1863.9247 \\ 1895.7634 & 2320.9296 & 2618.0257 \\ 2717.3310 & 2731.5887 & 2790.0143 \\ 2894.3641 & 2926.2492 & 2937.3791 \\ 2964.3828 & 2995.4980 & 3001.5022 \\ 3029.5969 & 3041.7400 & 3067.4680 \\ 3078.5978 & 3081.5576 & 3094.8741 \\ 3097.5821 & 3106.5931 & 3112.2715\end{array}$




\begin{tabular}{|c|c|c|}
\hline 3124.1367 & 3139.4989 & 3143.8231 \\
\hline 3156.5194 & 3170.4034 & 3181.6274 \\
\hline 3190.5342 & 3208.8914 & 3211.7270 \\
\hline 3220.6025 & 3221.9581 & 3230.6190 \\
\hline 3237.4885 & 3248.4948 & 3249.2594 \\
\hline 3255.5951 & 3256.6788 & 3264.3164 \\
\hline 3269.0520 & 3276.8967 & 3278.8036 \\
\hline 3281.4507 & 3284.8669 & 3292.7586 \\
\hline 3296.8924 & 3301.8274 & 3306.2411 \\
\hline 3314.0575 & 3317.8604 & 3322.7167 \\
\hline 3325.8866 & 3326.5315 & 3329.3632 \\
\hline 3332.9837 & 3337.2467 & 3339.5102 \\
\hline 3342.8974 & 3346.6696 & 3350.7381 \\
\hline 3360.4952 & 3377.5613 & 3382.6809 \\
\hline 3386.7781 & 3399.1932 & 3399.5642 \\
\hline 3408.2199 & 3410.7488 & 3419.6224 \\
\hline 3423.2761 & 3436.3044 & 3459.9827 \\
\hline 3469.1145 & 3473.6331 & 3494.5962 \\
\hline 3507.4135 & 3512.0593 & 3561.4252 \\
\hline \multicolumn{3}{|c|}{ Frequencies (not scaled) $\mathrm{C}-13\left(1^{\text {st }}\right.$ atom $) \mathrm{O} 18\left(2^{\text {nd }}\right.$ atom) } \\
\hline-3321.7938 & -1602.5736 & -1352.7809 \\
\hline-1226.6234 & -1204.1878 & -1166.5591 \\
\hline-1136.7764 & -1106.1909 & -1100.8690 \\
\hline-1098.7328 & -1093.7918 & -1091.7590 \\
\hline-1081.8756 & -1079.4400 & -1071.7076 \\
\hline-1069.1892 & -1063.0606 & -1060.7294 \\
\hline-1056.8573 & -1055.5292 & -1053.9385 \\
\hline-1052.0832 & -1045.6009 & -1042.9129 \\
\hline-1040.7900 & -1035.6328 & -1035.0563 \\
\hline-1031.2452 & -1028.8801 & -1025.1588 \\
\hline-1020.7177 & -1019.5210 & -1012.4680 \\
\hline-1006.5063 & -1005.3706 & -1000.5920 \\
\hline-999.7565 & -995.7019 & -992.3502 \\
\hline-989.6119 & -985.1147 & -979.5396 \\
\hline-974.7292 & -971.5524 & -969.1520 \\
\hline-964.2746 & -961.3122 & -958.3095 \\
\hline-952.9161 & -949.6572 & -945.4404 \\
\hline-941.4643 & -933.6500 & -931.0704 \\
\hline-929.5397 & -923.3216 & -918.9695 \\
\hline-917.3620 & -913.4962 & -910.1071 \\
\hline-907.5366 & -904.2206 & -897.7660 \\
\hline-896.0069 & -891.9799 & -890.2997 \\
\hline-885.3272 & -879.0960 & -876.0448 \\
\hline-869.7319 & -865.3411 & -858.4843 \\
\hline-851.2330 & -849.1822 & -846.6709 \\
\hline-842.2326 & -840.4001 & -838.0139 \\
\hline-830.3398 & -827.3889 & -823.9852 \\
\hline-817.2000 & -815.7139 & -810.9296 \\
\hline-805.8677 & -803.7276 & -798.9527 \\
\hline-795.8509 & -793.2899 & -779.8742 \\
\hline-774.0226 & -756.5602 & -748.7376 \\
\hline-716.1113 & -545.3386 & -497.2541 \\
\hline-458.8174 & -449.8199 & -442.4406 \\
\hline
\end{tabular}




$\begin{array}{lll}-439.2411 & -431.7129 & -424.5647 \\ -422.3336 & -419.0230 & -412.0560 \\ -411.0584 & -400.3031 & -391.4246 \\ -384.7508 & -381.5473 & -374.6187 \\ -373.6475 & -360.7159 & -355.4883 \\ -349.9691 & -347.8621 & -346.8953 \\ -343.8744 & -335.4790 & -327.8961 \\ -318.9473 & -311.9006 & -288.5280 \\ -263.9725 & -255.8087 & -253.6833 \\ -249.4043 & -248.1875 & -244.8018 \\ -242.5513 & -240.1067 & -234.6201 \\ -231.7937 & -222.6390 & -221.2674 \\ -209.5139 & -208.2482 & -201.5883 \\ -195.6181 & -193.7471 & -188.2037 \\ -184.6025 & -179.8213 & -165.0561 \\ -159.5186 & -148.4682 & -138.4002 \\ -131.3901 & -120.5264 & -108.7515 \\ -69.9368 & 25.4599 & 42.1784 \\ 48.5617 & 51.8280 & 56.3213 \\ 59.4718 & 62.8833 & 63.8997 \\ 68.2281 & 71.3196 & 74.0416 \\ 77.1158 & 80.3838 & 81.6289 \\ 84.2689 & 85.5523 & 87.8162 \\ 90.3528 & 91.0053 & 93.5559 \\ 95.2537 & 98.2024 & 100.6217 \\ 102.9325 & 106.4438 & 107.8452 \\ 108.7762 & 111.0601 & 111.8132 \\ 115.7303 & 117.6989 & 118.6149 \\ 122.7968 & 125.7039 & 128.5643 \\ 132.0715 & 133.6676 & 135.4958 \\ 139.1799 & 142.1769 & 152.9718 \\ 157.3032 & 158.1266 & 162.5917 \\ 162.9276 & 165.5784 & 169.9484 \\ 172.6094 & 175.0708 & 175.7332 \\ 177.8653 & 182.1490 & 185.5146 \\ 188.5904 & 189.0821 & 192.6284 \\ 200.1685 & 213.9516 & 216.4235 \\ 222.0398 & 226.5221 & 231.5629 \\ 234.7752 & 239.3940 & 244.1100 \\ 247.4928 & 255.5718 & 257.2366 \\ 260.1805 & 260.5881 & 264.2434 \\ 267.5870 & 279.6836 & 295.2527 \\ 296.3055 & 300.2905 & 302.4757 \\ 304.9282 & 309.8087 & 313.9808 \\ 318.0978 & 319.4625 & 323.9661 \\ 335.1939 & 341.0469 & 342.4220 \\ 349.0798 & 357.0571 & 361.8086 \\ 363.8422 & 369.5156 & 370.4760 \\ 375.0400 & 402.4664 & 414.6712 \\ 419.5719 & 424.4464 & 427.9765 \\ 430.6858 & 436.6147 & 437.7988 \\ 442.7282 & 447.7666 & 452.6454 \\ 455.3075 & 465.3999 & 470.7626\end{array}$




$\begin{array}{lll}484.7669 & 563.8630 & 628.6434 \\ 663.8365 & 671.8117 & 677.9925 \\ 681.5538 & 684.8509 & 694.0754 \\ 700.4660 & 702.6978 & 714.1325 \\ 716.7108 & 744.0554 & 752.9587 \\ 756.8886 & 767.7086 & 770.0484 \\ 777.8197 & 784.3103 & 788.2501 \\ 788.6228 & 795.8572 & 798.2623 \\ 806.0594 & 813.6817 & 816.0651 \\ 833.2750 & 840.2900 & 844.6989 \\ 875.2670 & 889.0368 & 898.5024 \\ 907.4545 & 921.2182 & 928.6538 \\ 942.3141 & 948.1511 & 952.8002 \\ 958.4678 & 964.3633 & 967.7660 \\ 973.1802 & 984.7098 & 989.1505 \\ 993.4491 & 995.5207 & 996.1533 \\ 999.9927 & 1006.6268 & 1009.9210 \\ 1011.3157 & 1013.0652 & 1021.7738 \\ 1024.4816 & 1035.0374 & 1038.2458 \\ 1040.8586 & 1043.3879 & 1046.4132 \\ 1047.1464 & 1048.6891 & 1059.5481 \\ 1062.4085 & 1065.3516 & 1073.1425 \\ 1074.5770 & 1079.7368 & 1083.7988 \\ 1095.8314 & 1107.8781 & 1127.3547 \\ 1137.6546 & 1172.2588 & 1175.7209 \\ 1197.5355 & 1205.1055 & 1214.3377 \\ 1218.9292 & 1219.4882 & 1232.5960 \\ 1245.5072 & 1251.1313 & 1253.9094 \\ 1258.0252 & 1262.3619 & 1273.0326 \\ 1280.3471 & 1284.2857 & 1289.9307 \\ 1293.7958 & 1298.4645 & 1303.7509 \\ 1307.7000 & 1318.2108 & 1354.5034 \\ 1376.4038 & 1387.3220 & 1422.2058 \\ 1442.4713 & 1445.0873 & 1453.4117 \\ 1484.5021 & 1517.7798 & 1548.6999 \\ 1563.1740 & 1592.4053 & 1596.6569 \\ 1623.9442 & 1640.4397 & 1641.8053 \\ 1657.8887 & 1664.3780 & 1674.8213 \\ 1676.4905 & 1679.3317 & 1691.9771 \\ 1692.4968 & 1700.1214 & 1707.9411 \\ 1717.4931 & 1735.3628 & 1739.1852 \\ 1740.8229 & 1744.1187 & 1748.1205 \\ 1749.3833 & 1756.0271 & 1763.1244 \\ 1767.2200 & 1769.3759 & 1775.1723 \\ 1787.5192 & 1788.5186 & 1791.3130 \\ 1802.8330 & 1804.6527 & 1863.9247 \\ 1895.7603 & 2320.9271 & 2618.0250 \\ 2717.3301 & 2731.5884 & 2790.0138 \\ 2894.3630 & 2926.2484 & 2937.3790 \\ 2964.3828 & 2995.4972 & 3001.5021 \\ 3029.5966 & 3041.7399 & 3067.4680 \\ 3078.5976 & 3081.5575 & 3094.8741 \\ 3097.5821 & 3106.5930 & 3112.2715\end{array}$




$\begin{array}{lll}3124.1365 & 3139.4988 & 3143.8230 \\ 3156.5194 & 3170.4033 & 3181.6268 \\ 3190.5342 & 3208.8912 & 3211.7270 \\ 3220.6024 & 3221.9581 & 3230.6190 \\ 3237.4885 & 3248.4948 & 3249.2593 \\ 3255.5951 & 3256.6787 & 3264.3163 \\ 3269.0520 & 3276.8966 & 3278.8036 \\ 3281.4507 & 3284.8669 & 3292.7586 \\ 3296.8924 & 3301.8272 & 3306.2410 \\ 3314.0574 & 3317.8604 & 3322.7167 \\ 3325.8866 & 3326.5314 & 3329.3631 \\ 3332.9836 & 3337.2466 & 3339.5102 \\ 3342.8974 & 3346.6695 & 3350.7381 \\ 3360.4951 & 3377.5613 & 3382.6809 \\ 3386.7781 & 3399.1932 & 3399.5641 \\ 3408.2199 & 3410.7488 & 3419.6224 \\ 3423.2761 & 3436.3044 & 3459.9827 \\ 3469.1145 & 3473.6331 & 3494.5962 \\ 3507.4135 & 3512.0593 & 3561.4252\end{array}$


Scientific Research Publishing (SCIRP) is one of the largest Open Access journal publishers. It is currently publishing more than 200 open access, online, peer-reviewed journals covering a wide range of academic disciplines. SCIRP serves the worldwide academic communities and contributes to the progress and application of science with its publication.

Other selected journals from SCIRP are listed as below. Submit your manuscript to us via either submit@scirp.org or Online Submission Portal.
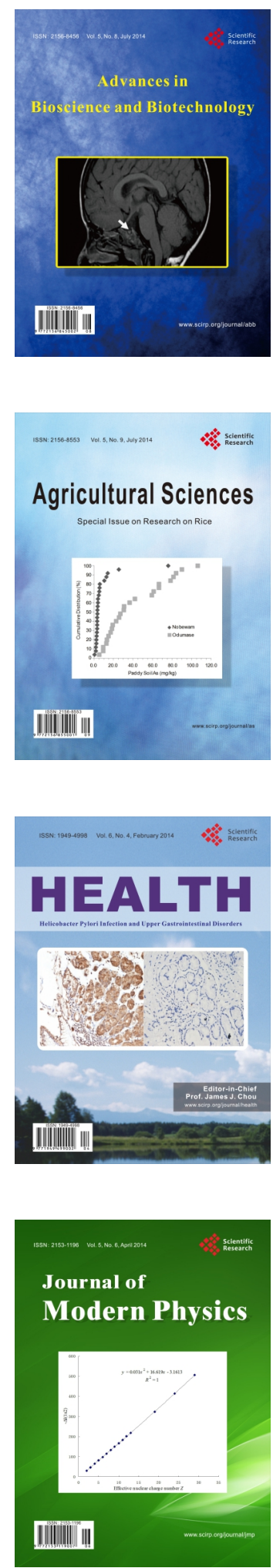
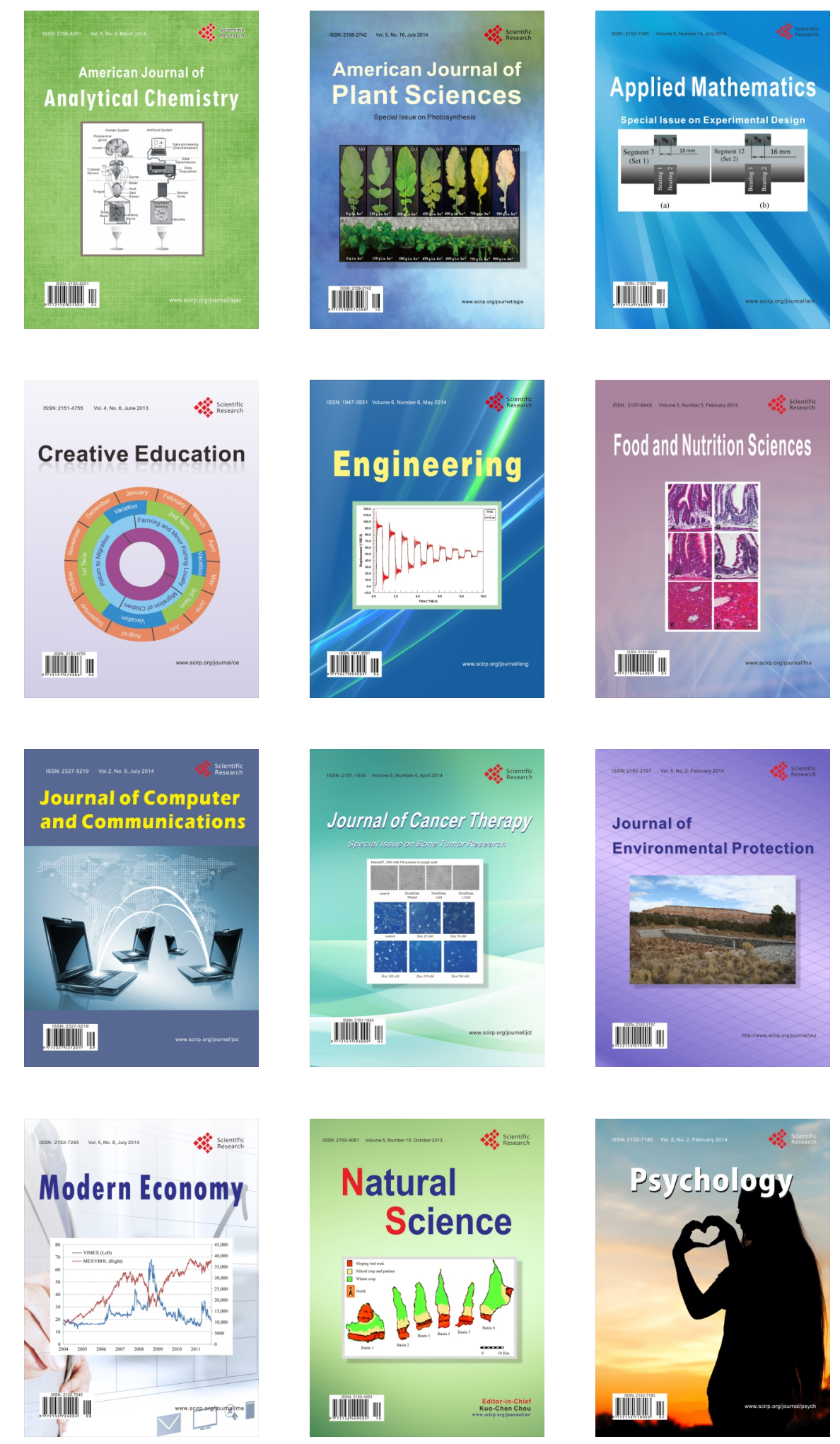